\title{
A Chemical Neural Network and Collective Behavior in Globally Coupled Oscillators
}

\author{
Fang Wang \\ West Virginia University
}

Follow this and additional works at: https://researchrepository.wvu.edu/etd

\section{Recommended Citation}

Wang, Fang, "A Chemical Neural Network and Collective Behavior in Globally Coupled Oscillators" (2011). Graduate Theses, Dissertations, and Problem Reports. 3383.

https://researchrepository.wvu.edu/etd/3383

This Dissertation is protected by copyright and/or related rights. It has been brought to you by the The Research Repository @ WVU with permission from the rights-holder(s). You are free to use this Dissertation in any way that is permitted by the copyright and related rights legislation that applies to your use. For other uses you must obtain permission from the rights-holder(s) directly, unless additional rights are indicated by a Creative Commons license in the record and/ or on the work itself. This Dissertation has been accepted for inclusion in WVU Graduate Theses, Dissertations, and Problem Reports collection by an authorized administrator of The Research Repository @ WVU.

For more information, please contact researchrepository@mail.wvu.edu. 


\title{
A Chemical Neural Network and \\ Collective Behavior in Globally \\ Coupled Oscillators
}

Fang Wang

DISSERTATION
Submitted to the Eberly College of Arts and Sciences at
West Virginia University in Partial Fulfillment of the Requirements for the Degree of
Doctor of Philosophy
in
Chemistry

\author{
Kenneth Showalter, Ph.D., Chairman \\ Charles Jaffé, Ph.D. \\ Michelle Richards-Babb, Ph.D. \\ Terry Gullion, Ph.D. \\ Larry Halliburton, Ph.D. \\ Morgantown, West Virginia \\ 2011
}

Keyword: BZ Reaction, Neural Network, Chemical Neural Network, Chemical Oscillators, Quorum Sensing, Kuramoto Synchronization, Phase Clusters

Copyright 2011 Fang Wang 


\title{
ABSTRACT
}

\section{A Chemical Neural Network and Collective Behavior in Globally Coupled Oscillators}

\author{
Fang Wang
}

The nervous system controls almost all actions in the body, and understanding its detailed structure and mechanism is one of the great challenges of science. Artificial neural networks have been modeled computationally to solve specific problems such as robot motion; however, few experimental studies have been designed to simulate biological neural networks because of the lack of experimental media with neural-like properties. An experimental network based on the photosensitive Belousov-Zhabotinsky reaction has been developed, in which the local excitability is controlled by light intensity. The spatiotemporal dynamics of these networks has been characterized, including sustained oscillations and collapse to the steady state. Here, we extend this work by incorporating the features of an actual network of neurons into the chemical system.

Many oscillatory systems exist in nature, and they can form collective behavior due to the interactions between them. The simplest collective behavior of oscillators is phase or frequency synchronization. Two distinct types of transitions, the quorum sensing transition and the Kuramoto transition to synchronization have been observed in a globally coupled 
oscillator system. For the Kuramoto transition to synchronization, the oscillators are gradually synchronized as the number density increases at low coupling strength. For the quorum sensing transition to synchronization, at high coupling strength, the oscillators are quiescent if the number density is lower than a critical value, and synchronized oscillations suddenly switch on as the number density reaches the critical value. We have studied populations of ferroin-coated catalytic particles and have characterized the two types of transitions to synchronization as a function of the population density and coupling strength of the oscillators with the surrounding solution.

Experimental studies of photochemical oscillators have shown more complex synchronization transitions compared to the ferroin-catalyzed oscillators. In this synchronization behavior, clusters of oscillators form in which frequency and phase are synchronized but with different phases for different phase clusters. Based on large populations of $\mathrm{Ru}(\mathrm{bpy})_{3}^{2+}$ catalyzed oscillators, we experimentally study the formation of phase clusters and their stability as a function of the density of the oscillators. We also simulate the cluster behavior based on the three-variable ZBKE model and compare our results with experiment. 


\section{Acknowledgements}

I sincerely appreciate my advisor, Dr. Kenneth Showalter, for his continuous support and encouragement during my graduate student life, providing me a great research atmosphere to finish my research. His great patience and broad knowledge lead me to solve many problems in my research. My special thanks to Dr. Mark Tinsley, his research capability, computer skill, and insights of experimental phenomena always inspired me and helped me to successfully achieve my aim. My deep appreciation goes to Dr. Annette Taylor whose brilliant ideas helped me achieve the important results of my research. I also want to express my gratitude to Dr. Eugene Mihaliuk, Dr. Zhaoyang Huang, and Hua Ke for their kindly help through my graduate student life, especially Dr. Eugene Mihaliuk for his help of reviewing my dissertation.

My greatest thanks go to my family: my parents, my sister and my brother, my husband and my lovely daughter, Morgan Cui. My husband, Hu Cui, for his courage and patience to always encourage me to conquer the problem of the work and move forward to the destination. 


\section{Contents}

Title Page

Abstract

1 The Belousov-Zhabotinsky Reaction 1

1.1 History of the Belousov-Zhabotinsky Reaction . . . . . . . . . . . . . . 1

1.2 The Mechanism of the BZ Reaction . . . . . . . . . . . . . . 2

1.3 The Kinetic Model: The Oregonator . . . . . . . . . . . . . . . 3

1.4 The Modified Oregonator Model . . . . . . . . . . . . . . 5

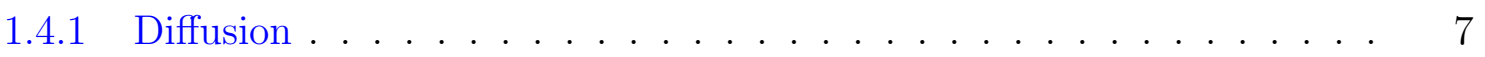

1.4.2 Light Sensitivity . . . . . . . . . . . . . . . . . 7

2 Neural Network $\quad 12$

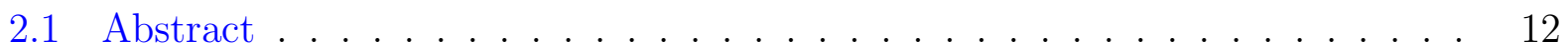

2.2 Networks . . . . . . . . . . . . . . . . . . . . . . . 13

2.2.1 Two Important Network Measures . . . . . . . . . . . . . . . . 13

2.2.2 Small-world Network . . . . . . . . . . . . . . . . . . 17

2.3 Neural Networks . . . . . . . . . . . . . . . . . . 17 
2.4 Two Important Activity Factors of Neural Networks . . . . . . . . . . . . . 26

2.5 Two Important Neural Network Models . . . . . . . . . . . . . . . . . 26

2.5.1 Integrate-and-Fire Model . . . . . . . . . . . . . . . 26

2.5.2 The Hebbian Plasticity Model . . . . . . . . . . . . . 27

3 Construction of a Chemical Neural Network 33

3.1 Design of The Network . . . . . . . . . . . . . . . . . . . . . . 34

3.2 Dynamic Construction of the Network . . . . . . . . . . . . . 35

3.3 Assigning the Links . . . . . . . . . . . . . . . . . . . . . . . . . . 37

3.4 Nature of the Links . . . . . . . . . . . . . . . . . . . . . . 38

3.4.1 The Distance Dependent Network Model . . . . . . . . . . . . . 38

3.4.2 The STDP Network Model . . . . . . . . . . . . . . . . . . 40

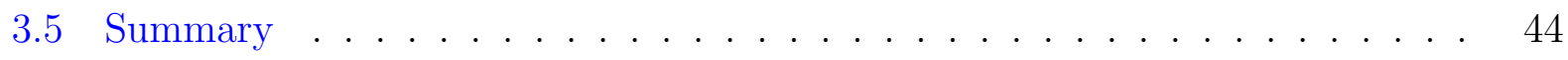

4 A Chemical Neuronal Network: Simulations and Experiments 50

4.1 Experimental System . . . . . . . . . . . . . . . . 50

4.1.1 Chemical System . . . . . . . . . . . . . . . 50

4.1.2 Chemical Waves in the BZ Reaction . . . . . . . . . . . . . 51

4.1.3 The Instrumental Setup . . . . . . . . . . . . . . . . 52

4.1.4 Design of the Illumination Pattern . . . . . . . . . . . . . . 52

4.2 Simulation Results . . . . . . . . . . . . . . . . . . 54

4.3 Experimental Results . . . . . . . . . . . . . . . . 55

4.4 Summary . . . . . . . . . . . . . . . . . . . 69 
5 Dynamical Quorum Sensing and Kuramoto Transitions to Synchronization 71

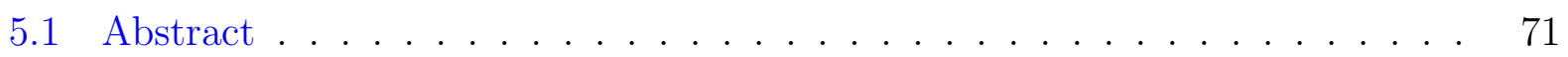

5.2 Experimental Setup . . . . . . . . . . . . . . . . 75

5.2.1 Catalyst Particle Preparation . . . . . . . . . . . . . 75

5.2 .2 Instrumental Setup . . . . . . . . . . . . . . . 76

5.2.3 The Optical Intensity of the Beads . . . . . . . . . . . . 78

5.3 Experimental Results . . . . . . . . . . . . . . . . 81

5.3.1 Kuramoto Transition to Sychronization . . . . . . . . . . . . . 81

5.3.2 Quorum Sensing Transition to Synchronization . . . . . . . . . . 83

5.4 Numerical Simulation . . . . . . . . . . . . . . . . . 84

5.4.1 ZBKE Model ........................ 84

5.4.2 The Relationship between Stirring Speed and

Chemical Exchange Rate . . . . . . . . . . . . . . . 89

5.4.3 A Model of the Globally Coupled Oscillatory Bead System . . . . . . 90

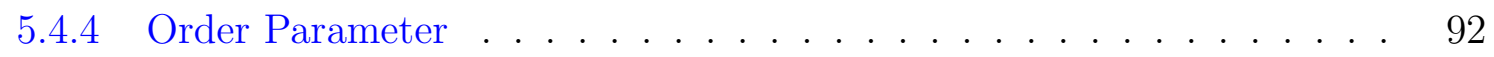

5.4.5 Simulation Results ....................... 93

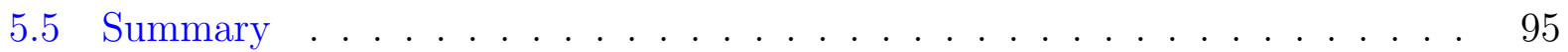

6 Phase Transition to Synchronization in Globally Coupled Oscillators 104

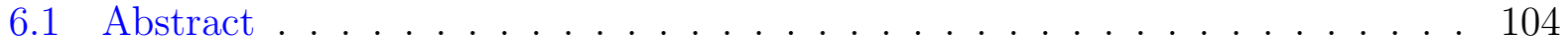

6.2 Experimental Setup . . . . . . . . . . . . . . . 106

6.2.1 Particle Preparation . . . . . . . . . . . . 106

6.2 .2 Instrumental Setup . . . . . . . . . . . . . . . . . . . 108 
6.3 Experimental Results . . . . . . . . . . . . . . . . . . . . . . . 109

6.4 Numerical Simulations . . . . . . . . . . . . . . . . . . . . . . . 111

6.4.1 ZBKE Model of Ruthenium Catalysed Particle System . . . . . . . 111

6.4.2 Comparison of Ruthenium and Ferroin Catalysed

BZ Reaction . . . . . . . . . . . . . . . . . . . . 113

6.4 .3 Formation of Clusters . . . . . . . . . . . . . . . . . . . . . . . 114

6.4.4 Factors Influencing Cluster Formation . . . . . . . . . . . . 117

6.5 Summary . . . . . . . . . . . . . . . . . . . . 120 


\section{List of Tables}

2.1 Two Network Measures of C. elegans . . . . . . . . . . . . . . . . . . 24

5.1 The parameter values in the ferroin-catalyzed ZBKE model $[14] \ldots$. . . . . 93

6.1 The parameter values in the ruthenium-catalyzed ZBKE model [13] . . . . 114 


\section{List of Figures}

1.1 The Nullclines of the Two-variable Oregonator Model in the $\mathrm{u}-\mathrm{v}$ Phase Plane 6

2.1 Regular Network and Its Clustering Coefficient . . . . . . . . . . . . . . 15

2.2 Three Typical Network Structures . . . . . . . . . . . . . . . . 16

2.3 Small-world Network . . . . . . . . . . . . . . . . . . . . . . 18

2.4 Scale-free Network . . . . . . . . . . . . . . . . . . . . . . . . 19

2.5 Neuron Structure . . . . . . . . . . . . . . . . . . . 20

2.6 Neuron Connections . . . . . . . . . . . . . . . . . . . . . . . . . . 21

2.7 Action Potential . . . . . . . . . . . . . . . . 22

2.8 C.elegans and Its Neural Network . . . . . . . . . . . . . . . . . . . . . 24

2.9 Cumulative Distributions of Connectivity . . . . . . . . . . . . 25

2.10 STDP Mechanism . . . . . . . . . . . . . . . . . . . 29

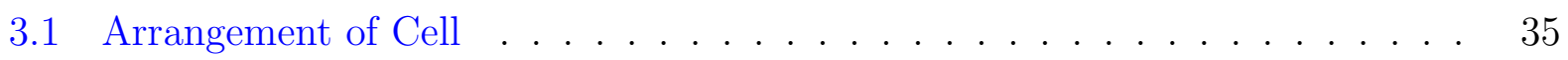

3.2 Average Path Length and Clustering Coefficient . . . . . . . . . . . 36

3.3 Simulation of the Cumulative Probability Distribution . . . . . . . . . 39

3.4 Working Process of the Cells . . . . . . . . . . . . . . 41

3.5 Potentiation and Depression ..................... 43 
3.6 The Probability of Extended Activity on a Network to Different Excitable Link Weight ....................... . . . 45

3.7 The Stable Distributions of Node Weight and Excitatory Link Weight . . . 46

4.1 Instrumental Setup . . . . . . . . . . . . . . . . . . . . . . 53

4.2 Experimental Design of the Gel . . . . . . . . . . . . . . . . . . 54

4.3 Simulation Result of 700 Links . . . . . . . . . . . . . . . 56

4.4 Simulation Result of 800 Links . . . . . . . . . . . . . . . . . . . 57

4.5 Simulation Results of the Activity . . . . . . . . . . . . . . . . 58

4.6 Experimental Results of One and Two Centers of Self-Sustained Static Pat-

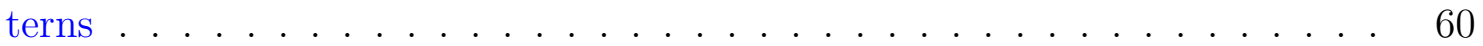

4.7 Experimental Results of Four and More Centers of Self-Sustained Static Pat-

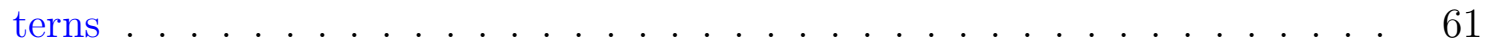

4.8 Analysis of Two Centers of Self-Sustained Static Patterns ～. . . . . . . . . 62

4.9 Analysis of One Center of Self-Sustained Static Patterns . . . . . . . . . . 63

4.10 Analysis of Four Centers of Self-Sustained Static Pattern . . . . . . . . . . . 64

4.11 Limited Restricted Unsteady Pattern . . . . . . . . . . . . . . . . 66

4.12 Restricted Unsteady Pattern _. . . . . . . . . . . . . . 67

4.13 Average Activity . . . . . . . . . . . . . . . . . . . . . 68

5.1 Dynamical Quorum Sensing and Kuramoto Synchronization . . . . . . . 74

5.2 Size and Oscillation Period Distributions of Beads . . . . . . . . . . . . 77

5.3 Experimental Setup . . . . . . . . . . . . . . . . . . . . 79

5.4 A Typical Plot of Average Intensity of the Beads in Oscillatory States . . . . 80 
5.5 Kuramoto Transition to Synchronization in Stirred System . . . . . . . . . . 82

5.6 Quorum Sensing Transition to Synchronization in Stirred System . . . . . 85

5.7 Snapshots of Bead Behavior . . . . . . . . . . . . . . 86

5.8 A Sketch of the Species Exchange . . . . . . . . . . . . . . . 91

5.9 Surface Plots of Bromous Acid Concentration and Order Parameter $K$. . . 96

5.10 Order Parameter and Period Distribution of the Beads in Kuramoto or Quorum Sensing Transition . . . . . . . . . . . . . . . . . . 97

5.11 Influence of Exchange Rate on the Transitions . . . . . . . . . . . . . . . 98

6.1 Image of Catalytic Micro-particles in Catalyst-free BZ Solution . . . . . . . . 107

6.2 Sampling Intensity Oscillations and Period Distribution . . . . . . . . . . 108

6.3 Instrumental Setup . . . . . . . . . . . . . . . . . . . . 109

6.4 Electrode Signals as Bead Density Increases . . . . . . . . . . . . . . . 111

6.5 Electrode Potential and Images . . . . . . . . . . . . . . . . . 112

6.6 Comparison of $\mathrm{Ru}(\mathrm{bpy})_{3}^{2+}$ and Ferroin-catalyzed BZ Solution . . . . . . . . 115

6.7 Simulations of Cluster Formation . . . . . . . . . . . . 116

6.8 Interpretation of Cluster Formation . . . . . . . . . . . . . . 118

6.9 Switching Oscillators . . . . . . . . . . . . . . . . . 119

6.10 Behavior as a Function of Number Density . . . . . . . . . . . . . . . . . . 121

6.11 Number of Clusters Versus the Coupling Strength . . . . . . . . . . . . . 122 


\section{Chapter 1}

\section{The Belousov-Zhabotinsky Reaction}

\subsection{History of the Belousov-Zhabotinsky Reaction}

Our research is based on a Belousov-Zhabotinsky (BZ) reaction. The BZ reaction was

first discovered by the Russian biochemist Boris Belousov in the early 1950s [1-3]. The work was not published, however, because the reaction he described was thought to violate the laws of thermodynamics. It was well known that all chemical reactions must proceed monotonically towards equilibrium; however, it was not realized that oscillations occurred in the intermediate species. In 1961, Zhabotinsky comfirmed the work of Belousov and brought it to light at an international conference in Prague in 1968 [4, 5]. The BZ reaction, as it came to be called, has become one of the most widely studied chemical reactions and is a model system for more complex biological and biochemical oscillatory systems. All of these oscillatory systems occur far from thermodynamic equilibrium. 


\subsection{The Mechanism of the BZ Reaction}

In 1972, Field, Körös and Noyes (FKN) first presented a detailed eleven step chemical mechanism for the BZ reaction, which has become known as FKN mechanism [6]. The BZ reaction consists of the bromate oxidation of malonic acid (MA) catalyzed by a metal ion or metal complexes in acidic aqueous solution. Overall the BZ reaction is given by $[1,2]$

$$
2 \mathrm{BrO}_{3}^{-}+3 \mathrm{CH}_{2}(\mathrm{COOH})_{2}+2 \mathrm{H}^{+} \rightarrow 2 \mathrm{BrCH}(\mathrm{COOH})_{2}+3 \mathrm{CO}_{2}+4 \mathrm{H}_{2} \mathrm{O}
$$

This reaction includes many elementary reactions with oscillations in the concentrations of many intermediates. There are three main processes $[1,7]$ : In the first process, a high concentration of bromide is consumed in two reactions, as $\mathrm{HBrO}_{2}$ is produced and consumed:

$$
\begin{gathered}
\mathrm{BrO}_{3}^{-}+\mathrm{Br}^{-}+2 \mathrm{H}^{+} \rightarrow \mathrm{HBrO}_{2}+\mathrm{HOBr} \\
\mathrm{HBrO}_{2}+\mathrm{Br}^{-}+\mathrm{H}^{+} \rightarrow 2 \mathrm{HOBr}
\end{gathered}
$$

Second, when bromide concentration becomes low enough, bromous acid $\left(\mathrm{HBrO}_{2}\right)$ is produced autocatalytically and the metal ion catalyst $\mathrm{M}_{r e d}$ is oxidized by supplying electrons for the process:

$$
\mathrm{BrO}_{3}^{-}+\mathrm{HBrO}_{2}+2 \mathrm{M}_{\mathrm{red}}+3 \mathrm{H}^{+} \rightarrow 2 \mathrm{HBrO}_{2}+2 \mathrm{M}_{\mathrm{ox}}+\mathrm{H}_{2} \mathrm{O} \text {. }
$$

The third process involves the reduction of the oxidized metal catalyst $\mathrm{M}_{o x}$ and the production of bromide:

$$
\mathrm{BrMA}+\mathrm{MA}+2 \mathrm{M}_{\mathrm{ox}} \rightarrow \mathrm{fBr}^{-}+2 \mathrm{M}_{\mathrm{red}}+\mathrm{gCO}_{2}+\mathrm{lH}^{+}
$$

Here, the stoichiometric factors $\mathrm{f}, \mathrm{g}$, and $\mathrm{l}$ are parameters that depend on the initial concentrations of MA. 


\subsection{The Kinetic Model: The Oregonator}

To describe experimental observations and predict conditions in which the characteristic oscillations of the BZ reaction might be observed, the most common model is the Oregonator, which was developed by Field and Noyes from the FKN mechanism [7].

In the Oregonator model, the FKN mechanism of the BZ reaction can be written as follows $[2,8]$ :

$$
\begin{gathered}
A+Y \stackrel{k_{1}}{\rightarrow} X+P, \\
X+Y \stackrel{k_{2}}{\rightarrow} 2 P, \\
A+X \stackrel{k_{3}}{\rightarrow} 2 X+2 Z, \\
2 X \stackrel{k_{4}}{\rightarrow} A+P, \\
B+Z \stackrel{k_{5}}{\rightarrow} h Y .
\end{gathered}
$$

Here $k_{1}, \ldots, k_{5}$ are the rate constants for the five steps, and the chemical species are identified by the following [9]:

$$
\begin{array}{lll}
A=\left[\mathrm{BrO}_{3}^{-}\right], & B=[\mathrm{BrMA}]+[\mathrm{MA}], & P=[\mathrm{HOBr}], \\
X=\left[\mathrm{HBrO}_{2}\right], & Y=\left[\mathrm{Br}^{-}\right], & Z=\left[\mathrm{M}_{o x}\right] .
\end{array}
$$

The steps of mechanism are assumed to be irreversible, and the concentrations of the major reactants $\mathrm{A}$ and $\mathrm{B}$ are treated as constants. The intermediate species $\mathrm{X}, \mathrm{Y}$ and $\mathrm{Z}$ 
obey the following rate equations $[10,11]$ :

$$
\begin{aligned}
& \frac{d X}{d T}=k_{1} A Y-k_{2} X Y+k_{3} A X-2 k_{4} X^{2} \\
& \frac{d Y}{d T}=-k_{1} A Y-k_{2} X Y+h k_{5} B Z \\
& \frac{d Z}{d T}=2 k_{3} A X-k_{5} B Z .
\end{aligned}
$$

In order to replace $\mathrm{X}, \mathrm{Y}, \mathrm{Z}$ and $\mathrm{T}$ with non-dimensional variables, the following definitions are used, which were introduced by Tyson and Fife [9, 12]:

$$
\begin{aligned}
u & =\left(\frac{2 k_{4}}{k_{3} A}\right) X, & v & =\left(\frac{k_{4} k_{5} B}{\left(k_{3} A\right)^{2}}\right) Z, \\
w & =\left(\frac{k_{2}}{k_{3} A}\right) Y, & \epsilon & =\frac{k_{5} B}{k_{3} A}, \\
\epsilon^{\prime} & =\frac{2 k_{4} k_{5} B}{k_{2} k_{3} A}, & q & =\frac{2 k_{1} k_{4}}{k_{2} k_{3}}, \\
t & =k_{5} B T, & f & =2 h,
\end{aligned}
$$

where $\mathrm{u}, \mathrm{w}, \mathrm{v}$ and $\mathrm{t}$ are dimensionless variables related to $\mathrm{X}, \mathrm{Y}, \mathrm{Z}$ and $\mathrm{T}, \epsilon, \epsilon^{\prime}$ and $\mathrm{q}$ are scaling parameters, and $\mathrm{f}$ is the stoichiometry parameter. Through substitution, the rate equations become

$$
\begin{gathered}
\epsilon \frac{d u}{d t}=q w-u w+u-u^{2}, \\
\frac{d v}{d t}=u-v \\
\epsilon^{\prime} \frac{d w}{d t}=-q w-u w+f v
\end{gathered}
$$

This is the 3 -variable Oregaonator model. Because $\epsilon^{\prime}<<\epsilon<<1$, w changes rapidly in time 
and can be treated as in a steady-state [10, 12], giving the algebraic relation

$$
w=\frac{f v}{u+q}
$$

Substituting this result into the above equations yields

$$
\begin{gathered}
\frac{\partial u}{\partial t}=\frac{1}{\epsilon}\left[u-u^{2}-(f v) \frac{u-q}{u+q}\right], \\
\frac{\partial v}{\partial t}=u-v .
\end{gathered}
$$

This two-variable Oregonator model facilitates the construction of the $u-v$ phase plane, which qualitatively describes the behavior of the BZ reaction [9, 10, 13]. Figure 1.1 shows three different intersections of the $u$-nullcline and $v$-nullcline based on three different values of $f$. The curve and staight lines in the figure represent the $\frac{\partial u}{\partial t}=0$ and $\frac{\partial v}{\partial t}=0$ nullclines, respectively. The crossing point of the two nullclines represents the steady state of the

system. If the intersection point lies on the left or the right branch of the $\frac{\partial u}{\partial t}=0$ nullcline curve, the system will return to the steady state after a perturbation. If the intersection is on the middle branch of the $u$-nullcline, the system is unstable because, after a small perturbation, a limit cycle is formed, as shown in Fig. 1.1(b).

\subsection{The Modified Oregonator Model}

The Oregonator model is based on the homogeneous BZ reaction; however, in many expentments, there are additional features that should be considered. In the following section, we will consider the modifications necessary to accomodate a light-sensitive catalyst as well as diffusion in the reaction medium. 


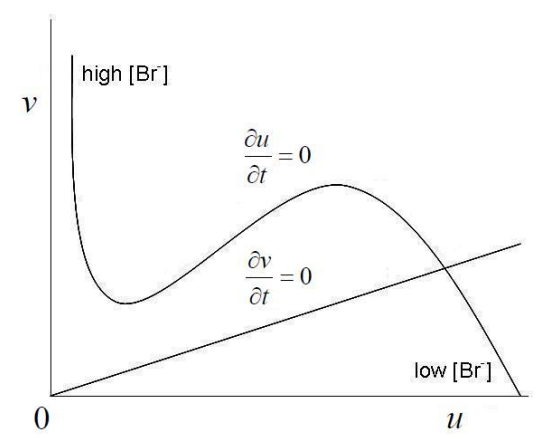

(a)

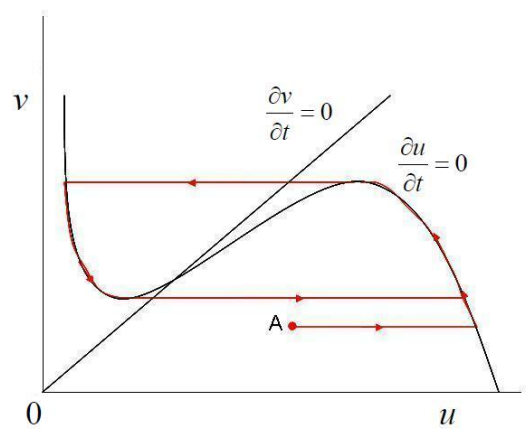

(b)

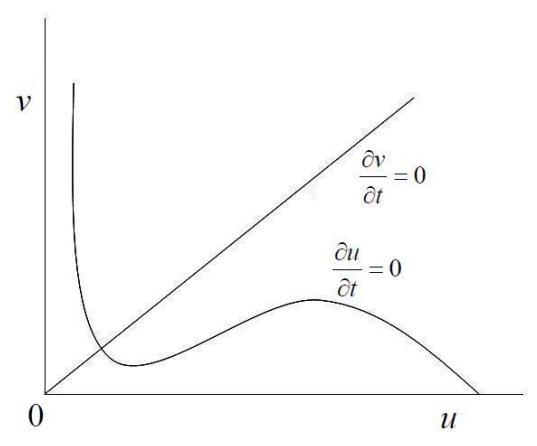

(c)

Figure 1.1. The nullclines of the two-variable Oregonator model in the $u-v$ phase plane. The cubic solid curve is the $u$-nullcline and the straight solid line is the $v$-nullcline. For (a) $f=0.25$, (b) $f=1$, the red line represent the limit cycle. A is an arbitary initial point, and the system first approaches the $u$-nullcline branch, moves alone the curve, until it jumps horizontally to the other branch, closing the loop. (c) $f=3$. [7, 11] 


\subsubsection{Diffusion}

The two-variable Oregonator model describes the BZ reaction in a homogeneous medium; however, when diffusion occurs, Laplacian terms must be incorporated into the equations [9]:

$$
\begin{gathered}
\frac{\partial u}{\partial t}=D_{u} \nabla^{2} u+\frac{1}{\epsilon}\left[u-u^{2}-(f v) \frac{u-q}{u+q}\right], \\
\frac{\partial v}{\partial t}=D_{v} \nabla^{2} v+u-v .
\end{gathered}
$$

Here, $D_{u}$ and $D_{v}$ are the dimensionless diffusion constants of $u$ and $v$, and $\nabla^{2}$ is the Laplacian operator. In the model, the concentrations of the reactants $\mathrm{BrO}_{3}^{-}$and $\mathrm{CH}_{2}(\mathrm{COOH})_{2}$ are constant. Continuously supplying fresh reactants maintains these concentrations constant [14].

The system is open, with the reaction restricted to a thin gel, which is surrounded by catalyst-free reactant solution. The catalyst is immobilized in the gel, which is supplied with constant concentrations of reactants. There are normally two diffusion terms required in the two-variable Oregonator model; however, in our system, the catalyst is stationary in the gel and diffusion of $v$ does not occur. The Oregonator with diffusion therefore becomes

$$
\begin{gathered}
\frac{\partial u}{\partial t}=D_{u} \nabla^{2} u+\frac{1}{\epsilon}\left[u-u^{2}-(f v) \frac{u-q}{u+q}\right], \\
\frac{\partial v}{\partial t}=u-v .
\end{gathered}
$$

\subsubsection{Light Sensitivity}

The photosensitive BZ reaction uses a light-sensitive catalyst. Five different species have been used as the photosensitive $\mathrm{BZ}$ catalyst, $\mathrm{Ce}^{3+}, \mathrm{Mn}^{2+}$, iron-phenantroline (also known as ferroin), $\mathrm{Ru}(\mathrm{bpy})_{3}^{2+}[1,15]$ and potassium ferrioxalate $\left(\mathrm{K}_{3} \mathrm{Fe}\left(\mathrm{C}_{2} \mathrm{O}_{4}\right)\right)$ with dipyridyl [16]. The 
$\mathrm{Ru}(\mathrm{bpy})_{3}^{2+}$ catalyzed BZ reaction is the most commonly studied version due to the specific photosensitive properties of the catalyst [17]. Light at $450 \mathrm{~nm}$ is absorbed by $\mathrm{Ru}(\mathrm{bpy})_{3}^{2+}$ and it is converted into the excited state $\mathrm{Ru}(\mathrm{bpy})_{3}^{* 2+}$. The excited state of the complex is a strong reducing agent, which reduces bromomalonic acid to $\mathrm{Br}^{-}$[18]. Its mechanism has been studied in detail and a kinetic model has been constructed [19]:

$$
\begin{aligned}
\frac{\partial u}{\partial t} & =D_{u} \nabla^{2} u+\frac{1}{\epsilon}\left[u-u^{2}-(\phi+f v) \frac{u-q}{u+q}\right], \\
\frac{\partial v}{\partial t} & =u-v .
\end{aligned}
$$

Here $\phi$ represents the light intensity. In the oscillatory regime, the excitability of the system is increased by decreasing the light intensity and decreased by increasing the light intensity. If the light intensity is increased above a certain threshold, it will suppress the oscillations. 


\section{Reference}

[1] R. Field and M. Burger, Oscillations and traveling waves in chemical systems (John Wiley \& Sons, Princeton, NJ, 1985). 1, 2, 7

[2] I. R. Epstein and K. Showalter, "Nonlinear chemical dynamics: Oscillations, patterns, and chaos," J. Phys. Chem. 100, 13132-13147 (1996).

Online Version 2, 3

[3] B. P. Belousov, "A periodic reaction and its mechanism," Sbornik Referatov po Radiatsionni Meditsine, 145 (1958), republished in English in reference [1]. 1

[4] A. Zhabotinsky, "Periodic processes of the oxidation of malonic acid in solution," Biofizika 9, 306 (1964). 1

[5] A. Zhabotinsky, "Periodic liquid-phase oxidation reactions," Dokl. Akad. Nauk SSSR 157, $392(1964) .1$

[6] R. J. Field, E. Körös, and R. M. Noyes, "Oscillations in chemical systems. I. detailed mechanism in a system showing temporal oscillations," J. Am. Chem. Soc. 94, 13941396 (1972).

Online Version 2

[7] R. J. Field and R. M. Noyes, "Oscillations in chemical systems. IV. limit cycle behavior in a model of a real chemical reaction," J. Chem. Phys. 60, 1877-1884 (1974). Online Version 2, 3, 6 
[8] A. S. Mikhailov and K. Showalter, "Control of waves, patterns and turbulence in chemical systems," Phys. Rep. 425, 79-194 (2006).

Online Version 3

[9] J. Tyson and P. Fife, "Target patterns in a realistic model of the Belousov-Zhabotinsky reaction," J. Chem. Phys. 73, 2224-2237 (1980).

Online Version 3, 4, 5, 7

[10] J. Tyson, "Scaling and reducing the Field-Körös-Noyes mechanism of the BelousovZhabotinsky reaction," J. Chem. Phys. 86, 3006-3012 (1982).

Online Version 4, 5

[11] S. K. Scott, Oscillations, Waves, and Chaos in Chemical Kinetics (Oxford University Press, New York, 1994). 4, 6

[12] H. J. Krug, L. Pohlmann, and L. Kuhnert, "Analysis of the modified complete Oregonator accounting for oxygen sensitivity and photosensitivity of Belousov-Zhabotinskii systems," J. Phys. Chem. 94, 4862-4866 (1990).

Online Version 4, 5

[13] F. Chirila, Feedback control of wave propagation patterns in excitable media, Ph.D. thesis, West Virginia University (2003). 5

[14] S. Kádár, J. Wang, and K. Showalter, "Noise-supported traveling waves in sub-excitable media," Nature 391, 770-772 (1998).

Online Version 7 
[15] M. Varga, E. Körös, and G. Putirskaya, Nonlinear Phenomena in Chemical Dynamics (Springer, Berlin, 1981). 7

[16] L. Kuhnert and H. Linde, "Signal-antwort-verhalten von autokatalytischen reaktionsystemen," Z. Phys. Chemie. 260, 423-434 (1979). 7

[17] K. Kalyanasundram, "Photophysics, photochemistry and solar energy conversion with tris(bipyrirdyl) ruthenium(II) and its analogues," Coord. Chem. Rev. 46, 159-244 (1982). 8

[18] S. Kádár, T. Amemiya, and K. Showalter, "Reaction mechanism for light sensitivity of the $\mathrm{Ru}(\mathrm{bpy})_{3}^{2+}$ catalyzed Belousov-Zhabotinsky reaction," J. Phys. Chem. A 101, 8200-8206 (1997).

Online Version 8

[19] J. Cui, Synchronization of Spatiotemporal Patterns and Modeling Disease Spreading Using Excitable Media, Ph.D. thesis, WVU (2004). 8 


\section{Chapter 2}

\section{Neural Network}

\subsection{Abstract}

The nervous system is the key structure that controls the functioning of an animal. The basic building block of the nervous system is the neuron. While the electrochemical mechanism of an individual neuron is reasonably well understood [1], how these neurons self organize into large scale networks and how these network structures process and control behavior is a research area that is still in its infancy.

A system of neurons may be considered to be a network of excitable nodes, each of which can be stimulated to create a signal. [1,2]. A number of theoretical studies of networks of such excitable nodes have been conducted [3,4]. Recently, our group has studied an experimentally realizable network based on the photosensitive Belousov-Zhabotinsky reaction, in which the local excitability is controlled using light intensity [5]. Here, we propose to extend this work by incorporating the features of an actual network of neurons into the chemical system. A neural cell receives input from a multitude of other neurons. If the total input is 
greater than some threshold, the neuron will fire and send a signal to other neurons that are connected to it. This "integrate and fire" connective mechanism will be incorporated into our experimental chemical system.

A learning rule will also be introduced into the system, allowing evolution of the network. In neuronal tissue, links between neurons become stronger or weaker depending on the relative timing of their firing. A spike time dependent plasticity (STDP) learning algorithm will be used to simulate the evolution of a neuronal network.

\section{$2.2 \quad$ Networks}

Networks are common in many natural and artificial systems [6,7], such as the topology of a food web, the world-wide web, the internet backbone, and the neural network of the C. elegans worm [6]. A network consists of nodes and links. For different natural networks, the nodes may represent different units, and if there is a connection between two nodes, then a link is said to exist. The collection of these nodes and links constitutes the network.

\subsubsection{Two Important Network Measures}

Several important measures can be defined to characterize the structure of the network connections. In order to understand this structure, we begin with a simple network given in

Fig. 2.1. A regular 20 node network with each node having 4 connections to its neighbors is shown in (a) of Fig. 2.1. The clustering coefficient $C$ is the average fraction of neighbors 
of a node that are also neighbors of each other. If node $A$ has $z$ neighbors, then at most $\frac{z(z-1)}{2}$ links can exist between them. $C_{A}$ is the fraction of these links that actually exist. The clustering coefficient $C$ is the average of $C_{A}$ over all nodes in the network. Panel (b) of Fig. 2.1 shows the six possible links between the four neighbors of the node A in panel (a). Since the actual number of connections between these neighbors is three, $C_{A}$ of this node is $\frac{3}{6}=0.5$. Because this is a regular network, all the nodes have the same $C_{A}$ value. Then the clustering coefficient $C$ of this network is equal to $C_{A}=0.5$.

The second measure is the average length $\mathrm{L}$, which is defined as the average distance of the shortest path between any two nodes in the network. Fig. 2.2 shows three typical network structures. Fig. 2.2(a) is a regular 20 node network. Fig. 2.2(c) is a random network, where randomly chosen pairs of nodes are connected by links. A regular network typically has high values of $\mathrm{C}$ and $\mathrm{L}$, indicating a high connectivity between neighboring nodes but large average separation between nodes. A random network has low $\mathrm{C}$ and $\mathrm{L}$ values, indicating a low connectivity between neighboring nodes and small average separation between nodes [8]. The network shown in Fig. 2.2(b) is constructed by randomly rewiring a small fraction of the links of Fig. 2.2(a) to new nodes. This network has the important property of both a high clustering coefficient $\mathrm{C}$ and a low average distance L. This network was first developed by Watts and Strogatz and is termed a small-world network [9]. 


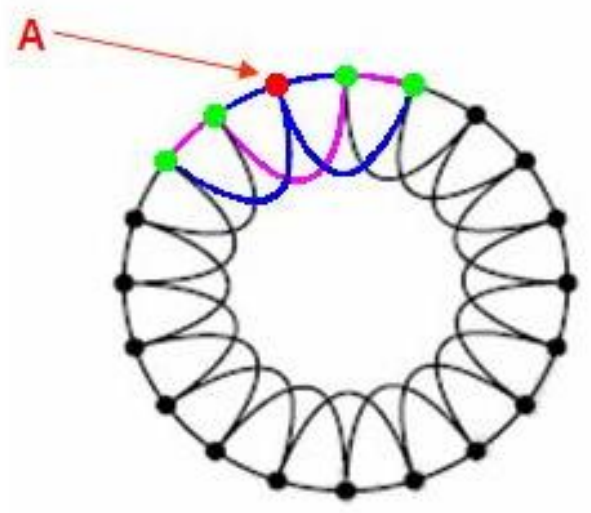

Regular

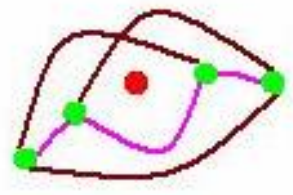

(b)

Figure 2.1. (a) A regular 20 node network with 4 connected neighbors for each node. The 4 green nodes are the neighbors of the red node A. (Modified from Fig. 2.2(a).) (b) All possible links which can exist between these 4 neighbor nodes, with the purple lines showing the links present in (a). 

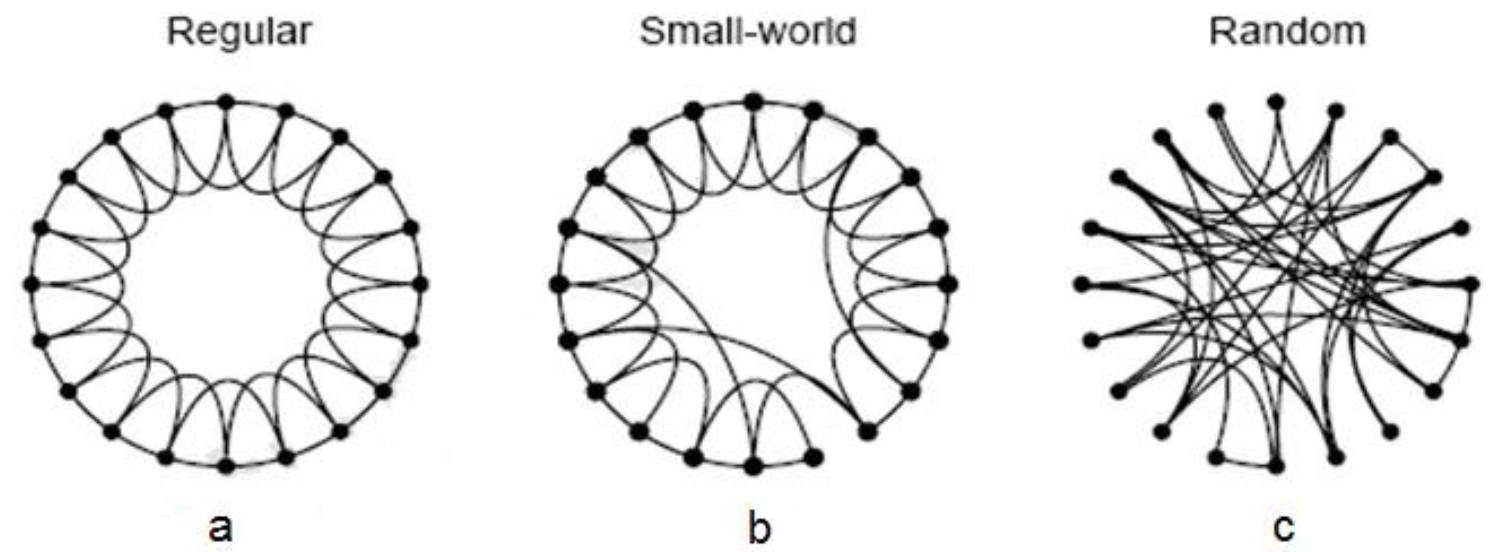

Figure 2.2. (a) Regular network, with nodes $\mathrm{n}=20$ and degree $\mathrm{k}=4$. (b) Randomly rewiring a small fraction of the links to new nodes produces a Watts-Strogatz small-world network. (c) Random network. (Figure from reference [9].) 


\subsubsection{Small-world Network}

Many real-world networks have been found to have a small-world network structure [9]. In a network, the number of links connected to each node $k$ is called the degree or the connectivity. In the Watts-Strogatz model of small-world networks, the probability distribution of the connectivity $P(k)$, defined as the fraction of nodes which have $k$ links, is similar to a Poisson distribution centered around a mean $k$ value of the network, $<k>$. The distribution is typically depicted as a cumulative distribution which exponentially decreases with $k$, as shown in Fig. 2.3. Another important type of small-world network is a scale-free network, which is identified according to its $P(k)$ distribution. This type of network occurs in numerous natural systems $[7,10]$, and $P(k)$ is found to decrease as a power-law of $k\left(P(k) \propto k^{-\gamma}\right)$, as shown in Fig. 2.4.

\subsection{Neural Networks}

The nervous system consists of two types of cells: neurons and glia. Any action of the brain, ranging from the simplest reflex to higher level functions, such as language, are all based on the same substrate: the neuron. It has been estimated that there are more than $10^{11}$ neurons in the human brain, many of which share the common features illustrated in Fig. 2.5. These neurons combine to form a very large and complicated neural network. In general, an individual neuron is composed of four typical regions [1]:

1. Soma: the cell body which is involved in metabolism of the cell. 


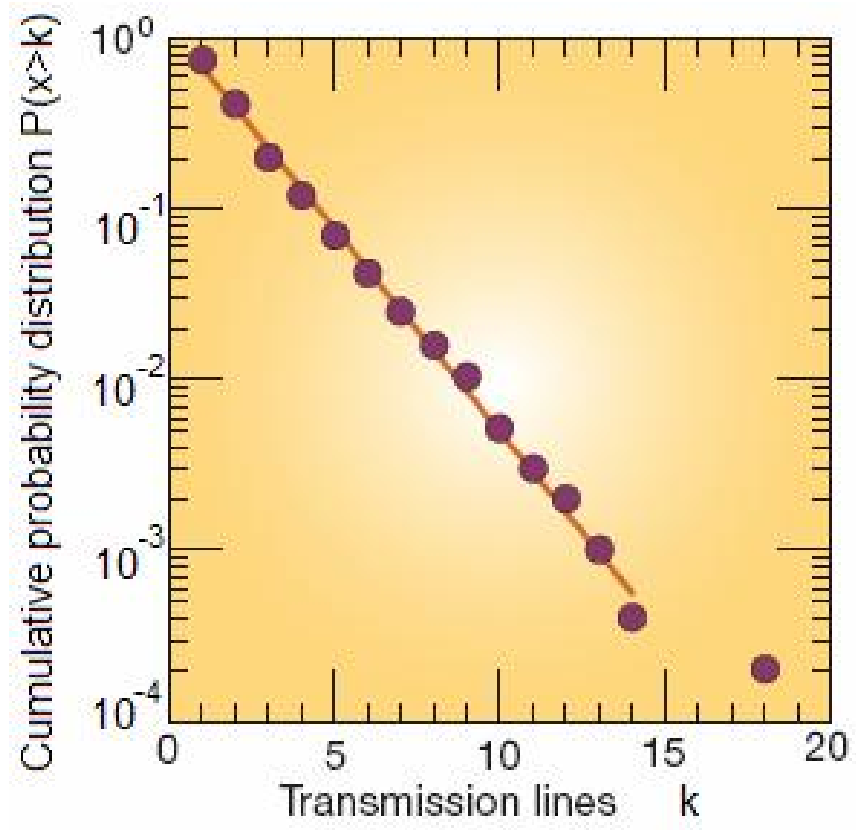

Figure 2.3. Cumulative probability distribution of the nodes versus transmission lines in a power grid network of Canada and the Western United States. Generators, substations and transformers are the nodes, and high-voltage transmission lines between them are the links of the power grid network. The plot shows that the log value of the probability distribution of nodes, which have at least $k$ transmission lines, linearly decays with the number of transmission lines $k$. (Figure from reference [6].) 


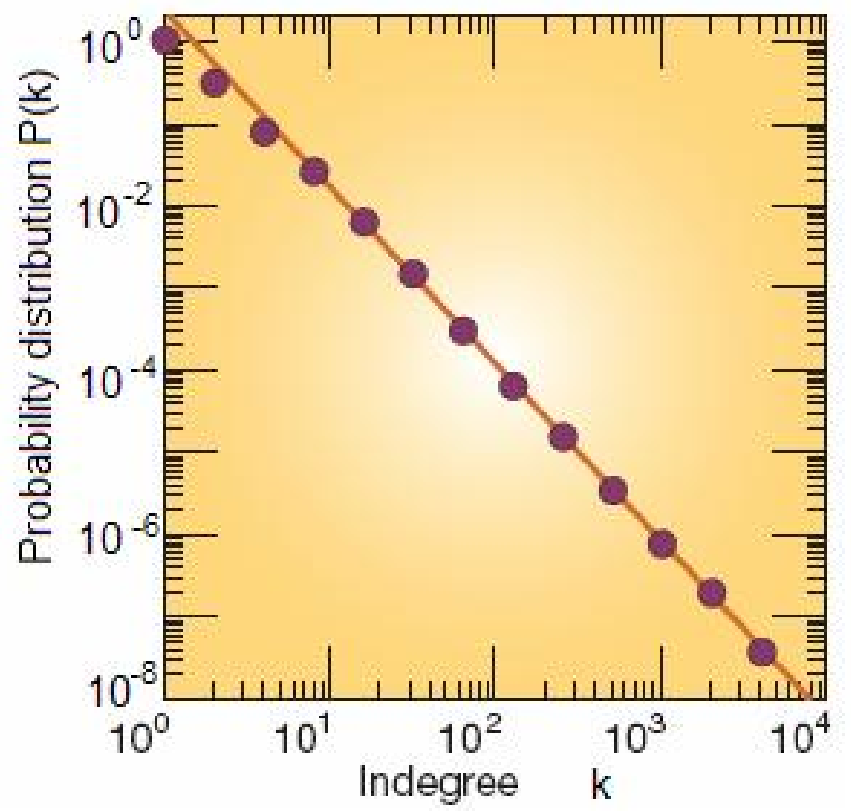

Figure 2.4. Degree distribution of the world-wide web. Nodes represent web pages; links represent URL (Uniform Resource Locater) hyperlinks between these pages. The figure shows that the distribution for the proportion of web pages versus the number of incoming links (indegree $k$ ) approximately follows a power law, and with an exponent $\gamma \approx 2.2$. (Figure from reference [6].) 


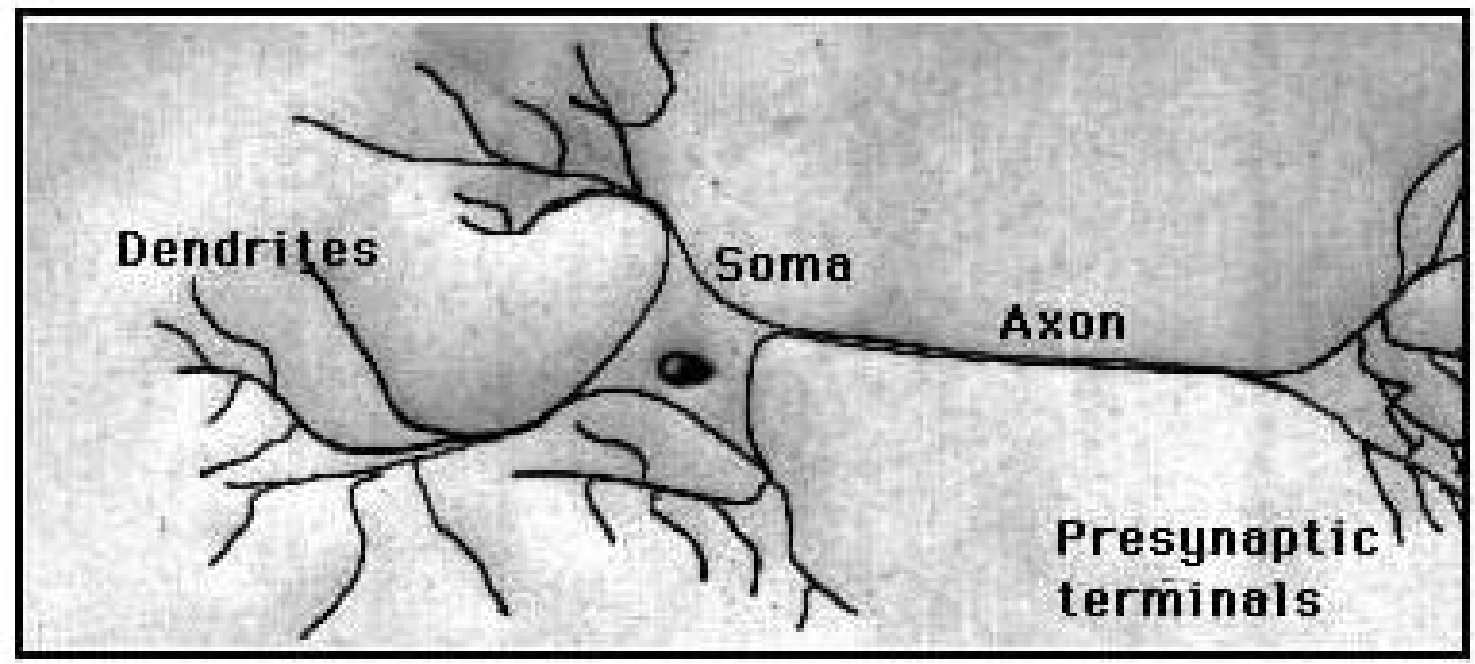

Figure 2.5. Structure of a neuron. (Figure from reference [1].)

2. Axon: the output and conducting structure of the neuron. Each neuron has only one axon. The axon arises from the axon hillock, where a signal may be initiated if the neuron has received a certain threshold stimulus.

3. Presynaptic terminals: branch structures that are located at the end of an axon.

4. Dendrites: branch structures that receive input signals, establishing connections with other neurons. 


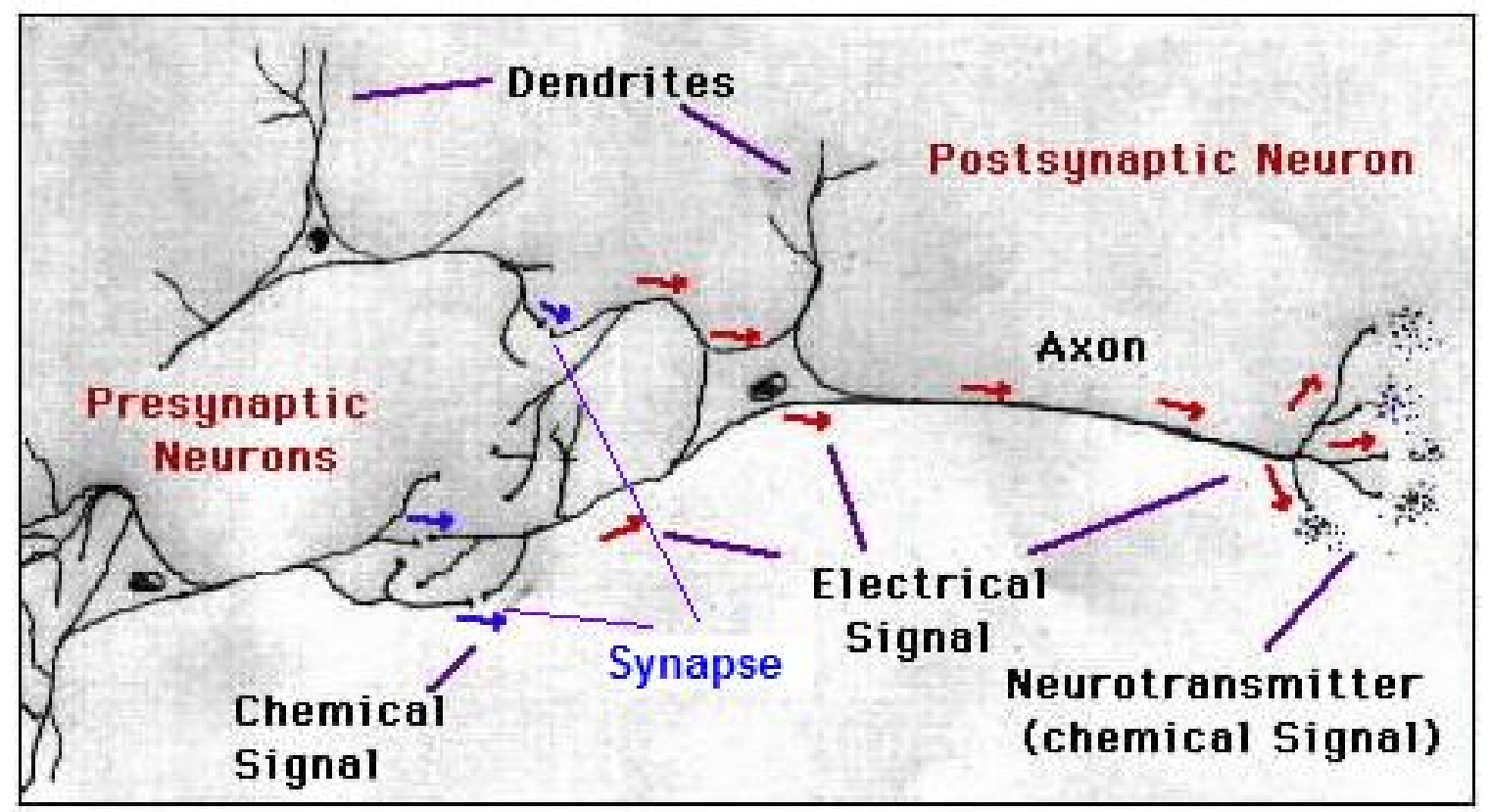

Figure 2.6. Neuron connections. (Figure from reference [1].)

The neuron transmits information through its presynaptic terminals to the dendrites of other neurons. The point of connection between two neurons is called a synapse. The presynaptic neuron is the neuron sending information out and the postsynaptic neuron is the neuron receiving information, as shown in Fig. 2.6. The collection of neurons, which are linked by their terminals and dendrites, constitutes a neuronal network [1].

The nature of the signal transmission within and between neurons is complex. Neurons maintain an electrical potential difference across their outer membrane. This is caused by concentration differences of ions inside the cell and the surrounding liquid. The input 


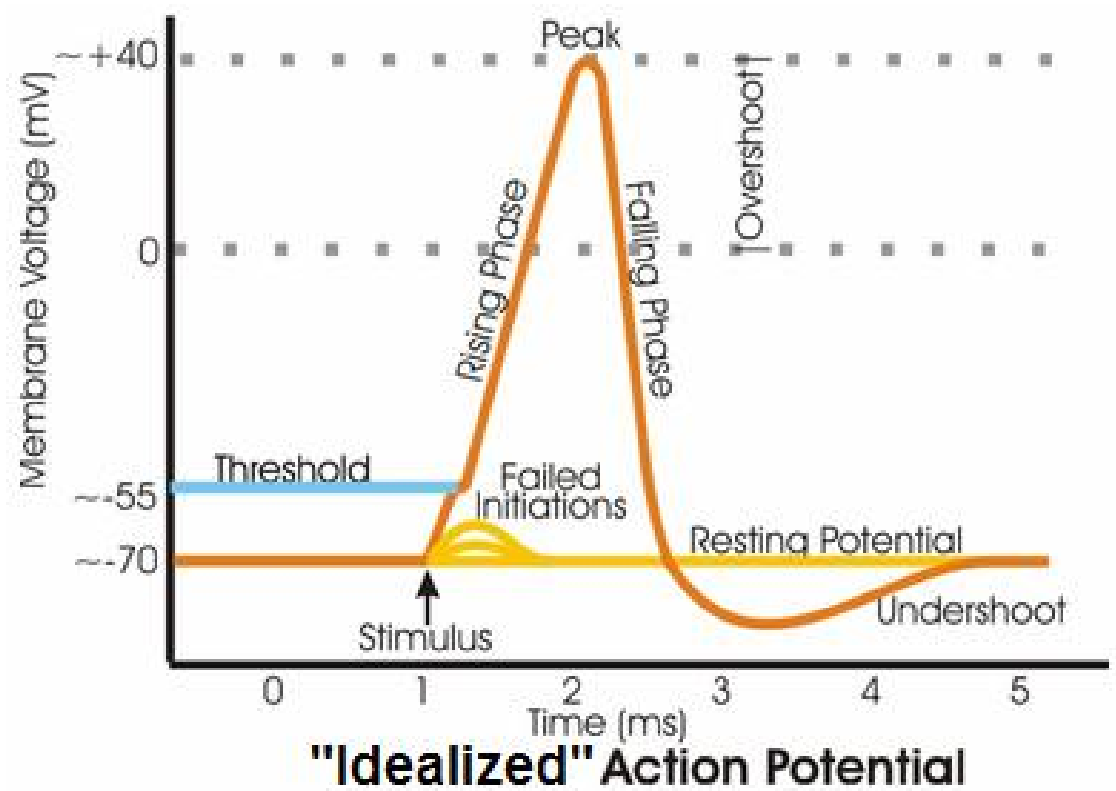

Figure 2.7. Various phases of an idealized action potential as it goes through the cell membrane of a neuron. (Figure from reference [11].)

synaptic potentials to a neuron, which are caused by the outputs of presynaptic neurons, are integrated and give rise to one or more action potentials if the sum of the input synaptic potentials is higher than a certain threshold [1]. The action potential is a wave of electrical discharge that travels along the membrane of a cell. It can be created by many types of cells; here we are concerned with a "typical" neuronal cell, as shown in Fig. 2.7 [11]. A neuron may be thought of as an "excitable oscillator" that is capable of integrating its synaptic potential and firing when this potential is above a certain threshold [2].

Biological neurons self-assemble to form complicated neural networks. The study of the structure and function of these biological neural networks is still in its infancy $[2,12]$. One 
network that has received much attention is that of a small worm called Caenorhabditis elegans, shown in panel (a) of Fig. 2.8 [13]. Owing to its simple nervous system, all the connections of this network have been elucidated [14]. Treating each neuron as a node and each connection as a link, the nervous system of $C$. elegans consists of 282 nodes and 2,335 unique links. Panel (b) of Fig. 2.8 represents the real neural network which is made of these nodes and links in the $C$. elegans neural system[10]. Table 2.1 shows two important measures of this neural network $(L$ and $C$ ), which indicates that it has the properties of a small-world network.

The probability distribution of the connectivity $P(k)$ is found to lie somewhere between that of a Watts-Strogatz network and a scale-free network. Figure 2.9 shows the relationship between the probability distribution of the connectivity $P(k)$ and the degree $k$ of the $C$. elegans nervous system. This neural network consists of only 282 neurons (nodes), and in order to reduce the effects of noise on this small data set, the data are plotted as a cumulative distribution. From Fig. 2.9B, we see that the connectivity probability distribution is not a power law distribution, nor does it show a clearly exponential distribution in graph C $[10,15]$. Therefore, this neural network has neither a scale-free network structure nor a Watts-Strogatz structure. For this reason, it is referred to as C. elegans structure [10]. 


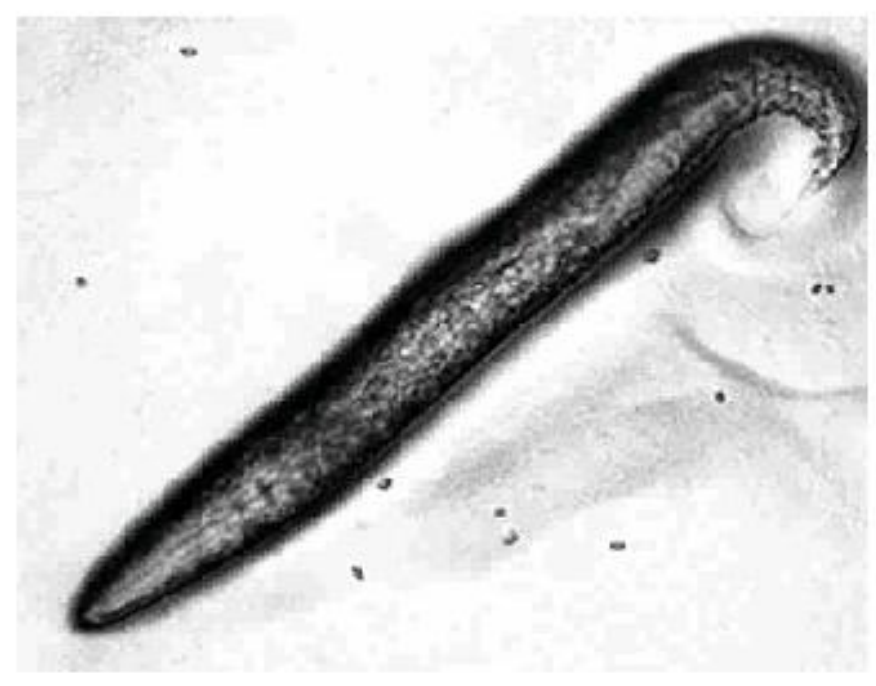

(a)

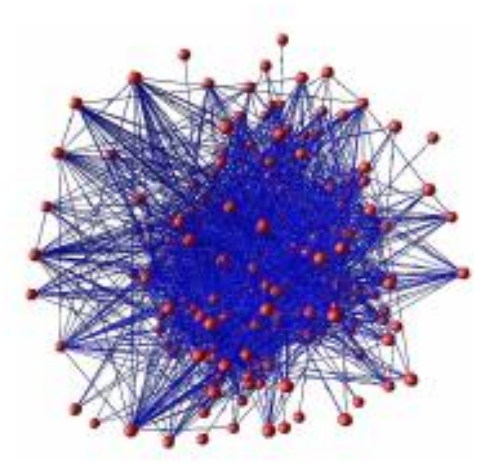

(b)

Figure 2.8. (a) A magnified picture of $C$. elegans (actual size is approximately $1 \mathrm{~mm}$ ). (Figure from reference [13].) (b) A simple model to illustrate the C. elegans neural network. Red nodes represent neurons and links show the connections between them. Spatial positions are placed randomly. (Figure from reference [10].)

\begin{tabular}{|l|c|c|c|c|}
\hline & $L_{\text {actual }}$ & $L_{\text {random }}$ & $C_{\text {actual }}$ & $C_{\text {random }}$ \\
\hline C.elegans & 2.65 & 2.25 & 0.28 & 0.05 \\
\hline
\end{tabular}

Table 2.1. The average length $L$ and clustering coefficient $C$ of the $C$. elegans neural network compared to a random network with the same number of nodes and average number of links per node $<k>: \mathrm{n}=282,<k>=14$. (Table from reference [9].) 

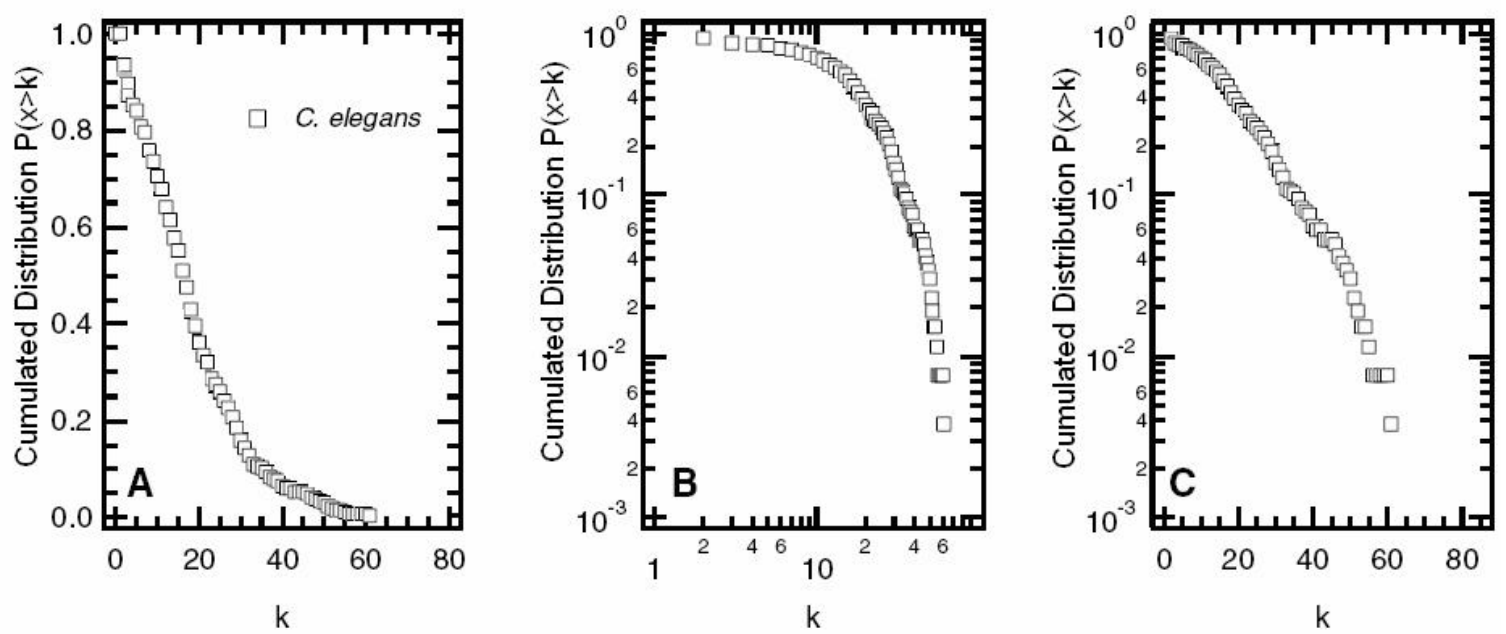

Figure 2.9. The cumulative distributions of connectivity $(P(x>k))$ versus link $k$, where $k$ is the number of links to and from each neuron for the $C$. elegans neural network. These three graphs plot the same data on different axes as (A) linear-linear, (B) log-log, (C) log-linear. (Figure from reference [10].) 


\subsection{Two Important Activity Factors of Neural Net- works}

In a neural network system, each neuron is connected to many other neurons by synapses, which act as links. The synaptic inputs may be excitory or inhibitory to the neuron. Excitatory links increase the activity of the neuron, while inhibitory links suppress the activity of the neuron. The synaptic weight (usually denoted by $w$ ) is used to describe the strength and type of the connection between two neurons. If $w>0$, the synaptic connection is an excitatory link, and if $w<0$, the synaptic link is an inhibitory link. Changing the synaptic weight of neurons can cause growth or decrease of activity in the neural network [16]. Balancing the extent of the inhibitory and excitatory links serves to maintain the network at an optimal level of activity [17].

\subsection{Two Important Neural Network Models}

\subsubsection{Integrate-and-Fire Model}

From Section 2.3, we know that each network neuron has a membrane potential $V_{i}$. In the integrate-and-fire model, the action potentials are generated by the following simple rule:

"An action potential occurs whenever the membrane potential reaches a threshold value $V_{t h}$. Immediately after that, the membrane potential is reset to a value $V_{\text {reset }} . "$ [16] This means that when the combination of all the input signals is over a certain threshold for a neuron, the neuron fires and then relaxes to its rest state. 


\subsubsection{The Hebbian Plasticity Model}

Changes in the synaptic connections between neurons are thought to contribute to memory storage. These changes are believed to occur according to the Hebbian plasticity model.

In 1949, D. O. Hebb hypothesized an important concept of how learning occurs [18]:

"When an axon of cell A is near enough to excite a cell B and repeatedly or persistently takes part in firing it, some growth process or metabolic change takes place in one or both cells such that A's efficiency, as one of the cells firing B, is increased."

A modern model incorporating Hebbian plasticity is known as the spike time dependent plasticity (STDP) model. In STDP, a synaptic weight changes only if the time of the preand postsynaptic spikes occur sufficiently close in time [2]. We treat the time ranges of preand postsynaptic spikes as synaptic windows. In a synaptic window, the synapse will be potentiated if a presynaptic potential occurs before the postsynaptic spike, while the synapse will be depressed if a presynaptic potential occurs after the postsynaptic spike.

A model of STDP development has been proposed by Rossum, Bi and Turrigiano [19]. The weighting changes of the synapse is calculated according to the STDP rule in the model. We use $W$ to represent the weight of the synaptic connection, $W \rightarrow W+W_{p}$ for potentiation and $W \rightarrow W+W_{d}$ for depression, where

$$
\begin{gathered}
W_{p}=C_{p} \times e^{-\Delta t / \tau_{S T D P}}, \\
W_{d}=-C_{d} \times W \times e^{\Delta t / \tau_{S T D P}} .
\end{gathered}
$$


Here, $\Delta t$ is the time difference between the synaptic action and the postsynaptic spike, $\tau_{S T D P}$ is the time constant for depression and potentiation, $C_{d}$ is the average amount of the depression after one pairing connection, and $C_{p}$ is the average amount of the potentiation after one pairing connection (see Fig 2.10). In the neuron system, the relative synaptic change is shown by the conductance change and it decreases exponentially within the time difference $\Delta t[19]$. 
a

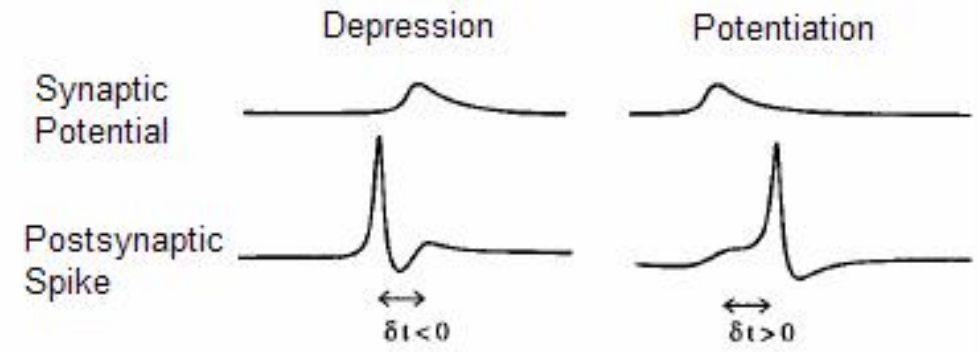

b

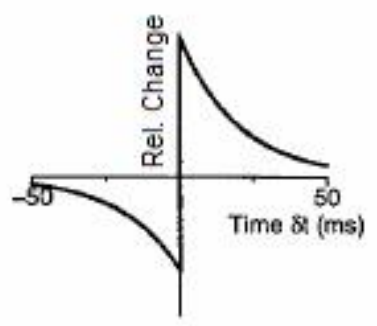

Weak Synapses

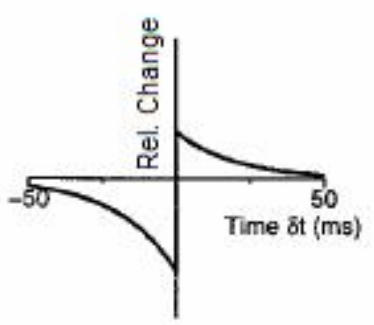

Strong Synapses

Figure 2.10. Schematic diagram of spike timing-dependent plasticity. (a) The depression and potentiation process. (b) The relative synaptic change versus the time difference between the synaptic potential and the postsynaptic spike. The relative synaptic change exponentially decreases with time, and the relative change for potentiation varies for weak or strong synapses, while the change for depression is not affected by the synaptic strength. (Figure from reference [19].) 


\section{Reference}

[1] H. Gutierrez and C. Ormsby, "A brief introduction to the brain: The neuron," Technical report, http://www.ifisiol.unam.mx/Brain/neuron.htm.

Online Version 12, 17, 20, 21, 22

[2] W. Gerstner and W. M. Kistler, Spiking Neuron Models (Single Neurons, Populations, Plasticity) (Cambridge University Press, 2002).

Online Version 12, 22, 27

[3] A. Roxin, H. Riecke, and S. Solla, "Self-sustained activity in a small-world network of excitable neurons," Phys. Rev. Lett. 92, 198101-1-4 (2004).

Online Version 12

[4] J. Pham, K. Pakdaman, and J. Vibert, "Noise induced coherent oscillations in randomly connected neural networks," Phys. Rev. E 58, 3610-3622 (1998).

Online Version 12

[5] A. J. Steele, M. Tinsley, and K. Showalter, "Spatiotemporal dynamics of networks of excitable nodes," Chaos 16, 015110-1-9 (2006).

Online Version 12

[6] S. Strogatz, "Exploring complex networks," Nature 410, 268-276 (2001). Online Version 13, 18, 19

[7] S. Boccaletti, V. Latora, Y. Moreno, M. Chavez, and D.-U. Hwang, "Complex networks: 
Structure and dynamics," Physics Reports 424, 175-308 (2006).

Online Version 13, 17

[8] M. E. J. Newman, "Models of the small world: A review," J. Stat. Phys. 101, 819-841 (2000).

Online Version 14

[9] D. Watts and S. Strogatz, "Collective dynamics of 'small-world' networks," Nature 393, 440-442 (1998).

Online Version 14, 16, 17, 24

[10] H. Berry and O. Temam, "Characterizing self-developing biological neural networks: A first step towards their application to computing systems," in "Lecture Notes in Computer Science," volume 3512, 306-317 (2005).

Online Version 17, 23, 24, 25

[11] J. H. Byrne, "Introduction to the action potential ( neuroscience online )," UT Houston Medical School, electronic neuroscience textbook.

Online Version 22

[12] C. Koch and G. Laurent, "Complexity and the nervous system,", Science 284, 96-98 (1999).

Online Version 22

[13] G. Williams, "Epileptic-like convulsions associated with lis-1 in the cytoskeletal control of neurotransmitter signaling in caenorhabiditis elegans," The University of Alabama 
(2004).

Online Version 23, 24

[14] K. Oshio, Y. Iwasaki, S. Morita, Y. O. S. Gomi, E. Akiyama, K. Omata, K. Oka, and K. Kawamura, "Database of synaptic connectivity of c. elegans for computation," Technical Report of CCeP, Keio Future No. 3, Keio University, Japan (2003).

Online Version 23

[15] S. Morita, K. Oshio, Y. Osana, Y. Funabashi, K. Oka, and K. Kawamura, "Geometrical structure of the neuronal network of caenorhabditis elegans," Physica A 298, 553-561 (2001).

Online Version 23

[16] T. P. Vogels, K. Rajan, and L. Abbott, "Neural network dynamics," Annu. Rev. Neurosci. 28, 357-376 (2005).

Online Version 26

[17] R. Segev, M. Benveniste, Y. Shapira, and E. Ben-Jacob, "Formation of electrically active clusterized neural networks," Phys. Rev. Lett. 90, 168101-1-4 (2003).

Online Version 26

[18] D. O. Hebb, The Organization of Behaviour (John Wiley \& Sons, New York, 1949). 27

[19] M. C. W. Rossum, G. Q. Bi, and G. G. Turrigiano, "Stable hebbian learning from spike timing-dependent plasticity," J. Neurosci. 20, 8812-8821 (2000).

Online Version 27, 28, 29 


\section{Chapter 3}

\section{Construction of a Chemical Neural Network}

A common way to understand the behavior of a complex system is through the analysis of

its individual elements. However, the overall system is often not a simple collection of the elements, even if we clearly understand the individual behavior of each, since their interactions are also important. Network theory supplies a useful tool to combine individual elements and their interactions together into an overall system. From the previous introduction to the network theory, it is clear that the dynamics of a network can be very complex even if the dynamics of its elements (nodes) is relatively simple. For example, in an artificial neural network, the structure and dynamics of a single element is simple; however, when multiple neurons connect into a neural network, the dynamics may be extremely complex. In this chapter, we describe the construction of a simple network based on an excitable medium that has dynamic properties similar to a collection of neurons, with the aim of exploring the characteristics of this network [1]. 


\subsection{Design of The Network}

The photosensitive BZ reaction has been used to model a spatiotemporal network of excitable nodes $[1,2]$. During the experiment, the gel is divided into square cells, with the light intensity in each cell controlled by the projector. Each cell is considered to be a node in the network. The network consists of an $n \times n$ array of cells and each cell is $m \times m$ pixels in size in a chessboard pattern (see Fig. 3.1). A wave can be initiated at a cell by setting the light intensity equal to zero at all grid points in the cell, while the surrounding medium is maintained in an excitable steady state. Local links arise by the propagation of reactiondiffusion waves from the initiation cells to their surrounding cells. Nonlocal links are created by changing the excitability by varying the light intensity of a destination cell according to the activity of a source cell. Prior to each experiment, a network of $\mathrm{N}$ nonlocal links is established by randomly choosing source cells and corresponding destination cells. The experimental system and numerical simulations have been used to investigate the dynamics of these simple networks. A number of dynamical behaviors have been observed, including sustained oscillation, period locking and phase locking of oscillators [1, 2].

The underlying network architecture of this experimental system can be examined using the average path length $L$ and the clustering coefficient $C$. For this network, the variations of the average path length $L$ and the clustering coefficient $C$ with the number of links are shown in Fig. 3.2. The statistical measures in this figure reveal that as the number of possible links increases, the average path length decreases, while the clustering coefficient remains close to the value of a regular network, categorizing it as a small-world network. The probability distribution of connectivity $P(k)$ is found to have an exponentially decreasing trend similar 


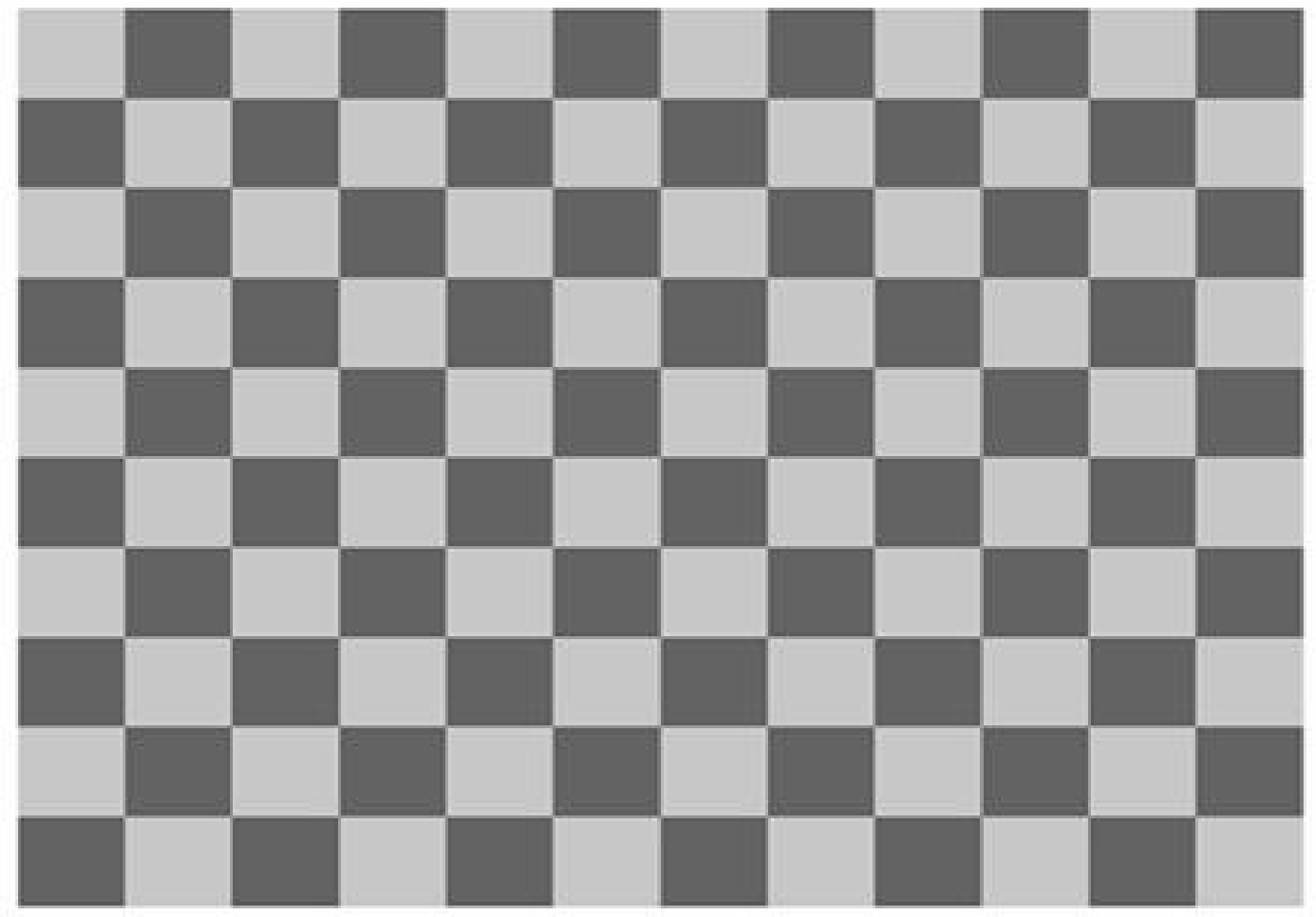

Figure 3.1. Construction of a network on an excitable medium. Each square represents a cell.

to the Watts-Strogatz model of a small-world network.

\subsection{Dynamic Construction of the Network}

We can impose a square array onto the gel, with the light intensity in each cell controlled by a projector in order to determine its excitability. In our previous work, there 


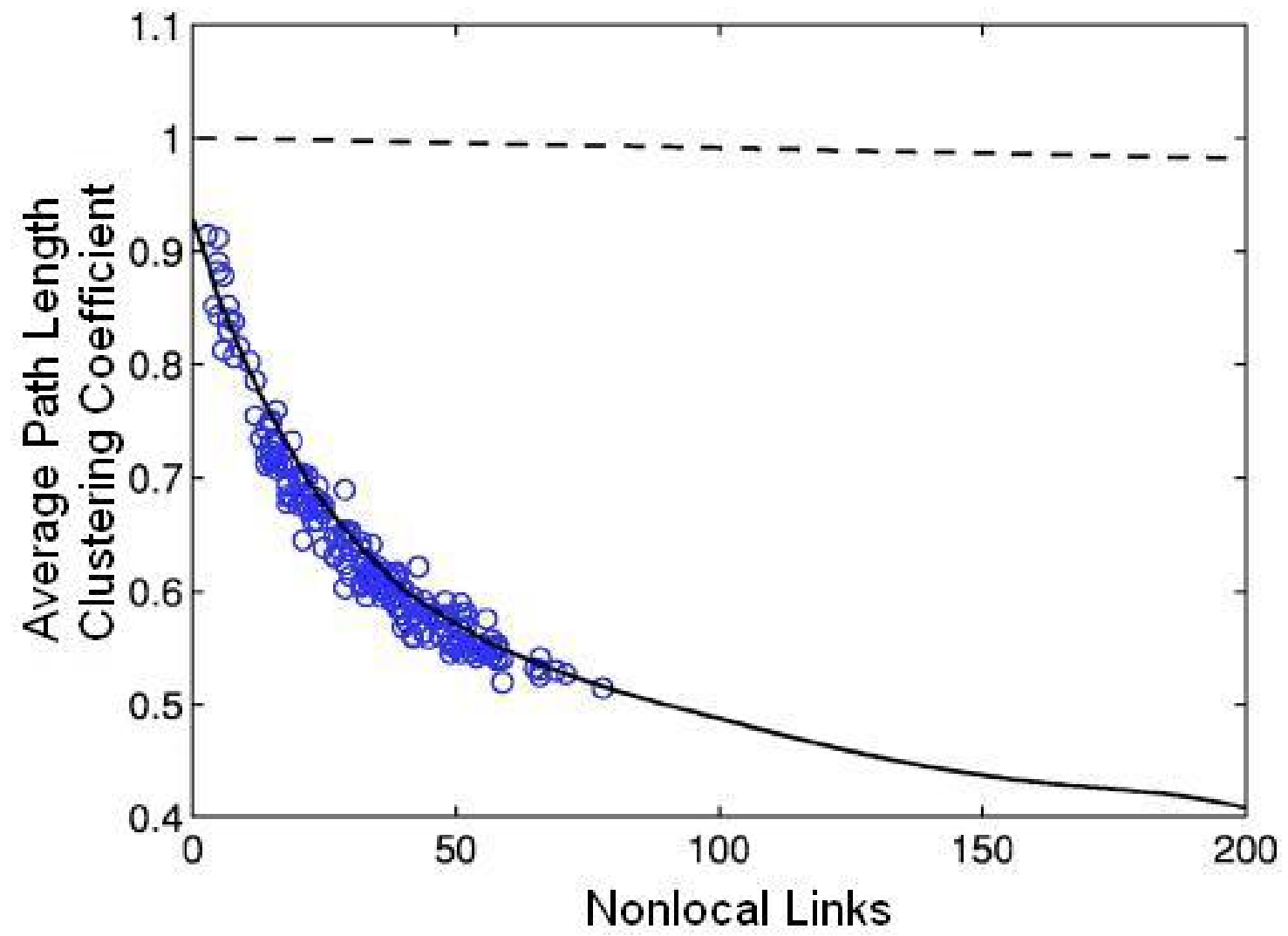

Figure 3.2. In the panel, the solid line represents the average path length and the dashed line represents the clustering coefficient. Both of them decrease with increasing the number of randomly assigned nonlocal links. The data in the figure are normalized by the average value of the regular network that has no nonlocal links. Circles are the normalized average path length data. (From reference [1].) 
were two types of links: local links and nonlocal links. Local links were initiated by the reaction-diffusion wave, and nonlocal links were assigned to random sites by the algorithm. In the work proposed here, all links are externally controlled. The reaction-diffusion wave is inhibited by surrounding each node with a barrier of high light intensity.

In the Oregonator model of the photosensitive BZ reaction, $D_{u}$ is the diffusion coefficient of $\mathrm{HBrO}_{2}$, which we set to zero in order to examine the ODE (ordinary differential equation) system without diffusion:

$$
\begin{gathered}
\frac{d u}{d t}=\frac{1}{\epsilon}\left[u-u^{2}-(\phi+f v) \frac{u-q}{u+q}\right], \\
\frac{d v}{d t}=u-v .
\end{gathered}
$$

For this two-variable Oregonator model, we use Euler method to solve $u$ and $v$ at time step $d t=0.001$ and the length of cell side $d x=0.15$. The parameters are $f=1.4, q=0.0002$, and $\epsilon=0.01$. The network is composed of a $12 \times 10$ cell array for the experiment, while a $50 \times 50$ cell array with each cell made up of $19 \times 19$ grid points is used for the simulation. The cell is excited and a wave is initiated in the cell when the light intensity $\phi$ is set to $\phi=$ 0 . The light intensity in the surrounding medium is maintained at $\phi=0.073$.

\subsection{Assigning the Links}

Studies have shown that neurons have a higher probability of being connected to neighboring neurons than distant neurons [3]. This distance dependence can be incorporated into our network by modifying the link probability distribution. 
We construct a link by choosing a source cell at random, and then choose a random destination cell at a distance $d$ with the probability $P(d)=1 / \xi \exp (-d / \xi)$. Here $\mathrm{d}$ is the Euclidian distance between the source cell and the target cell, and $\xi$ is a parameter determining the average connection distance [3]. If a destination cell with the calculated distance from the source cell does not exist, then a new choice is made.

Figure 3.3 shows a simulation of the cumulative probability distribution $P(x>k)$ corresponding to the connectivity based on the proposed model with $17 \times 17$ nodes and 2,335 links, where the linear-linear, log-log and log-linear plots versus the degree $\mathrm{k}$ are shown. We note that these plots are similar to the connectivity probability distribution of the C.elegans neural network. We will study the activity of the network both experimentally and computationally.

\subsection{Nature of the Links}

\subsubsection{The Distance Dependent Network Model}

In previous work, a link was initiated based on the activity of a source cell. If the excitation of the source cell was over a certain threshold, it triggered an increase in excitability of the destination cell, leading to wave initiation [1]. In the chemical neural network, every link between two nodes has an associated weight, and once the threshold is attained, the weight is maintained over the period. A cell reaches threshold when the sum of all inputs to that cell is larger than a predetermined value, leading to the excitation of the node. This, 

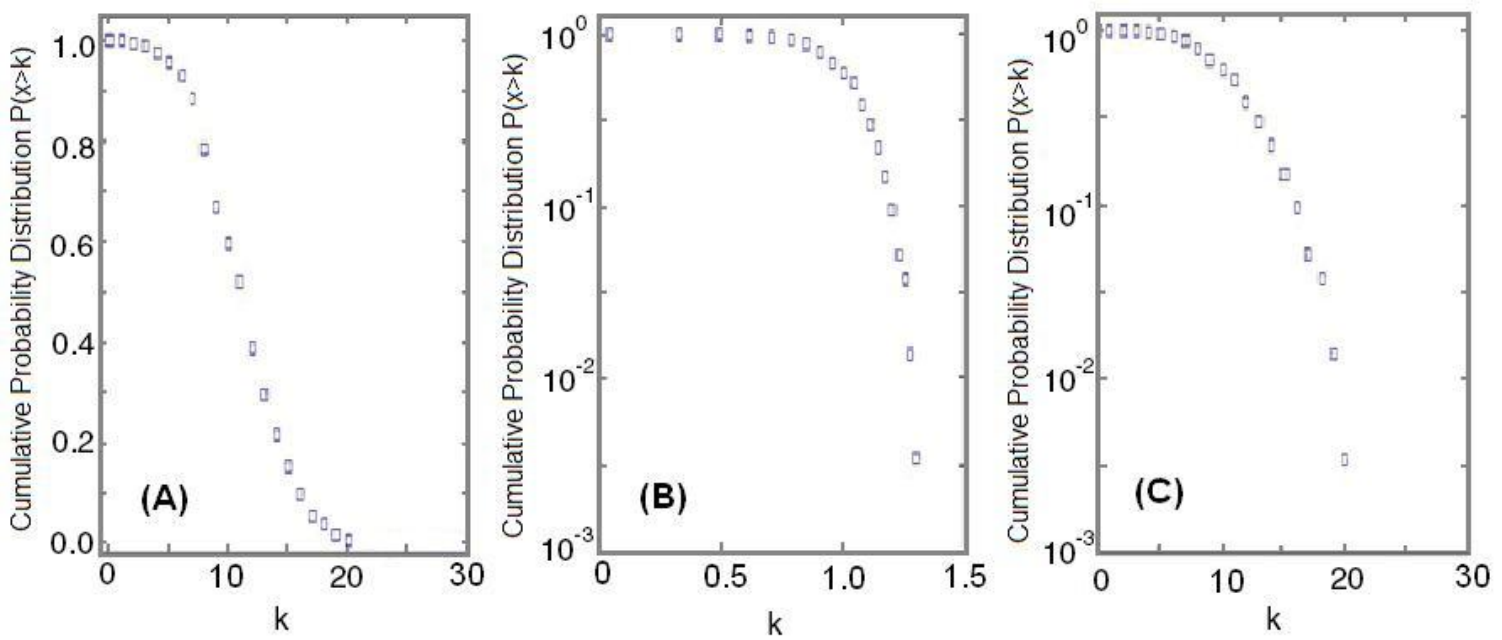

Figure 3.3. Cumulative distribution of the connectivity, $P(x>k)$, where $k$ is the number of links to and from each node. The same data are presented in (A) linear-linear, (B) log-log and (C) log-linear plots. Simulation carried out with a 17x17 node network and 2,335 links.

in turn, results in the transmission of excitation to other linked cells.

In the previous chapter, we described how light intensity effects the activity of the chemical medium. For the $\mathrm{Ru}(\mathrm{bpy})_{3}^{2+}$ catalyzed BZ system, the change of light intensity changes the dynamics of the destination cell from excitable to oscillatory. Once a reaction-diffusion wave appears in the source cell, the chemical neural system is initiated and the integrateand-fire model is applied.

From Section 2.5.1, we know that the synaptic weights of a neuronal network, based on the integrate-and-fire mechanism, are given by

$$
y_{i}=\sum_{j=1} w_{i j} x_{j} .
$$

Here, $y_{i}$ is the output signal from $i$ th neuron, $x_{j}$ is the input signal from the $j$ th neuron to $i$ th neuron, and $w_{i j}$ is the synaptic weight between neurons $i$ and $j$ [4]. In our system, the 
light intensity imposed on a cell reflects the weights of the links to that cell. A simplification in our model at this level is that all the active excitatory links have the same weights and all the active inhibitory links also have identical weights. The change of the light intensity at a cell is

$$
\Delta \phi=C_{e} w_{e}-C_{i} w_{i}
$$

where $C_{i}$ and $C_{e}$ are the number of the active inhibitory and excitatory links, and $w_{i}$ and $w_{e}$ are the weights of the inhibitory and excitatory links. The light intensity at the cell is

$$
\phi=\phi_{0}-\Delta \phi=\phi_{0}-C_{e} w_{e}+C_{i} w_{i}
$$

where $\phi_{0}$ is the background light intensity for all the cells. Decreasing the light intensity increases the activity of the BZ system.

Here we set the background light intensity of all cells $\phi_{0}=0.15$. After initiation, the active excitatory links will decrease the light intensity of the destination cells by 0.03 , while the active inhibitory links will increase the light intensity of the destination cells by 0.08 . The ratio of inhibitory to excitatory links is $1: 4$, which has been used in other neural network models $[5,6]$. Figure 3.4 shows a schematic representation of the integrate-and-fire process that is applied in our chemical network system.

\subsubsection{The STDP Network Model}

In Hebbian learning, the strength of the connections to a neuron is affected by the timing of neuronal spikes. Here, we implement the spike timing dependent plasticity (STDP) mechanism. This is implemented in the experimental photosensitive BZ system with the 


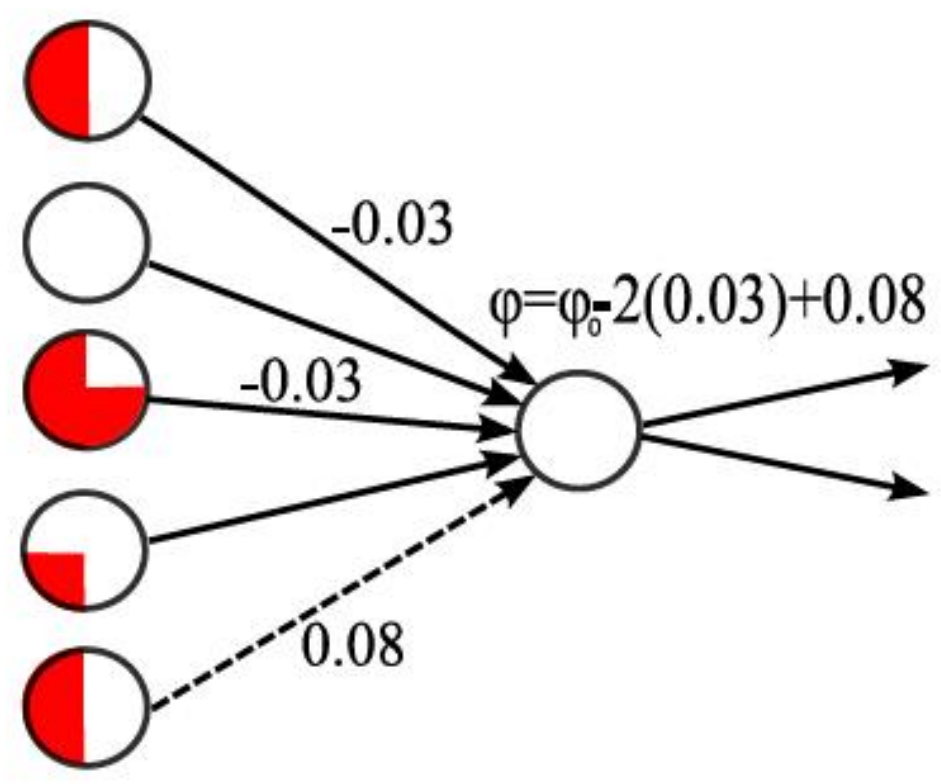

Figure 3.4. The circles represent the cells, and the shaded area represents the excitation coverage of the cell. When the excitation of a cell is $50 \%$ or more, the cell is defined as excited and the corresponding links are active (the first, third and fifth cells); otherwise, the cell is not excited and the corresponding links are not active (the second and fourth cells). Three cells are therefore excited and their links are active; two are excitatory links and decrease the light intensity at the destination cell, and one is an inhibitory link which increases the light intensity at the destination cell. The dashed line represents the inhibitory link and the solid lines represent excitatory links. The initial light intensity of the destination cell is given by $\phi_{0}$, and $\phi$ represents the light intensity that is imposed on the destination cell from the network. 
dependence of excitability on the light intensity. By changing the light intensity associated with a link (or the weight of a link), a potentiation or depression of the link can be simulated according to the STDP mechanism.

In the Oregonator model for the photosensitive BZ reaction, $\phi$ is the rate of photochemical bromide production and is proportional to the light intensity. The light intensity of a cell in its excitable steady state is initially $\phi=\phi_{0}$, with a weight $w=w_{0}$. After summing the $m$ currently active links for the destination cell $i$ in the network, the light intensity of that cell is given by

$$
\phi_{i}=\phi_{0}-\sum_{j}^{m} w_{j},
$$

where $w_{j}$ represents the value of the weight adjusted by the changes $w_{p}$ or $w_{d}$ of link $j$. The change in the potentiation weight $w_{p}$ and the change of depression weight $w_{d}$ are calculated from the equations in Section 2.5.2. The time difference between the excitation of a source and target cell is $\Delta t$, which is determined according to the time of the maximum value of $v$ in each cell (see Fig. 3.5). The value of $\Delta t$ determines whether the weight change corresponds to potentiation or depression. The time constant for depression and potentiation is $\tau_{S T D P}$, $c_{d}$ is the average extent of depression after one pairing connection, and $c_{p}$ is the average extent of potentiation after one pairing of connection. The illumination level is determined according to this algorithm, which is then imposed on the cell.

In order to maintain the activity of the network, a large number of links is required. Each link of the network is adjusted repeatedly based on the excitation of the source and destination cells. In our simulation model, the network is initially excited by decreasing the light intensity in the center cell of the network. If the combination of links from the initiated 

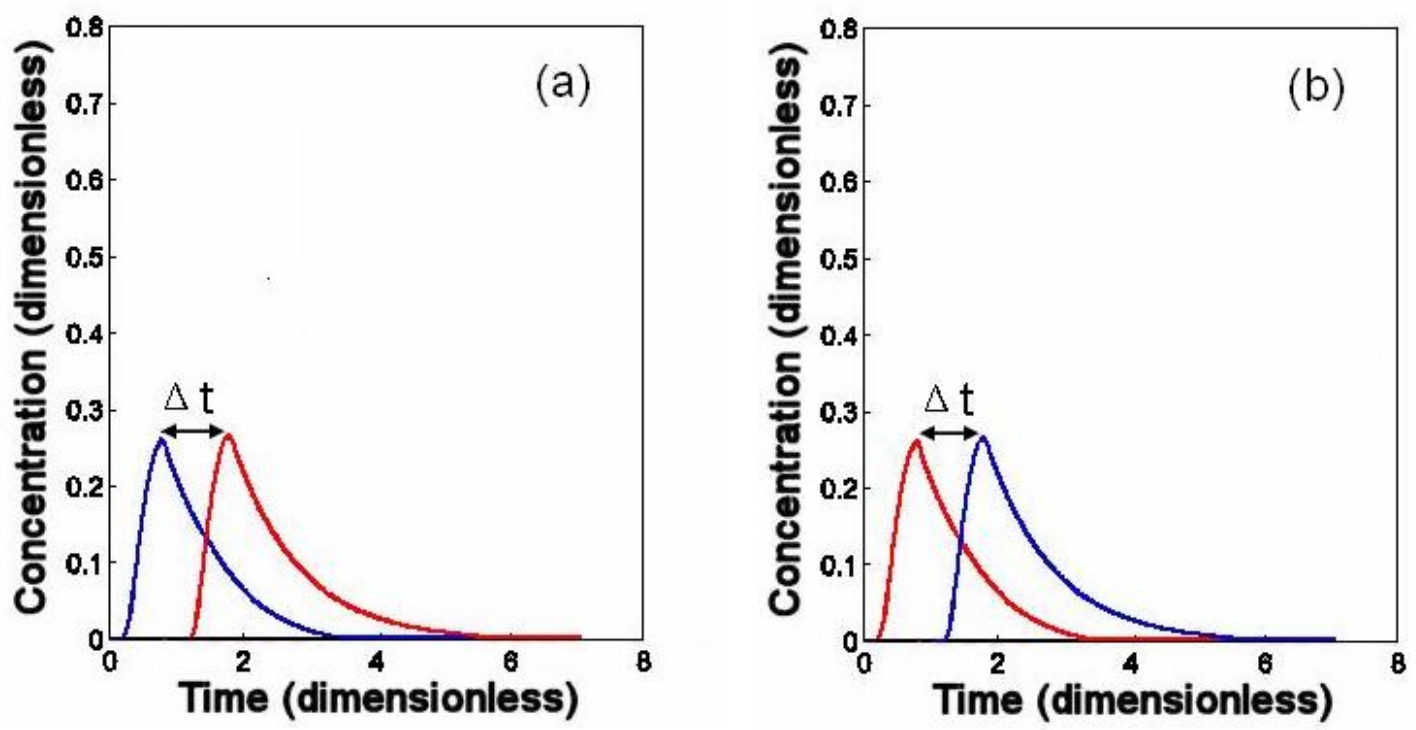

Figure 3.5. Potentiation or depression based on $\Delta t$, where potentiation is shown in (a) and depression is shown in (b). The blue and red lines show the dimensionless concentration $v$ for the source cell and target cell, respectively. In panel (a), the spike in the source cell is before of the spike of the target cell, $\Delta t>0$, and the weight of the link is therefore increased. In panel (b), the spike in the source cell is after that of the target cell, $\Delta t<0$, and the weight of the link is therefore decreased. 
cell results in wave activity, the activity on the network is sustained, otherwise, the activity on the network ceases. Adjusting the parameters of the network can change the activity of the network. The relevant parameters include the link weight and the number of links. In order to find the parameter regime in which the activity of the network will be sustained, the weight of the excitatory links is varied in the simulations. Figure 3.6 shows the probability of extended activity on the STDP network and the distance dependent network corresponding to different link weights [7].

In simulations, we find that $S T D P$ networks that maintain activity have a stable distribution of excitatory link weights and node weights. Figure 3.7 shows the stable distributions of excitable link weights and node weights in the STDP network.

\subsection{Summary}

We have studied a neural network using a cellular BZ reaction system. Although there are differences between neuronal networks and the BZ network, the BZ system is a useful model because its excitability is similar to that of a neuronal system.

The cells of the medium exhibit characteristics of the neural network elements. Two neural network models, the integrate-and-fire model and the STDP model, have been investigated. For these chemical networks, we have found that increasing the number of links increases the activity of the network until it becomes saturated with activity. In the distance dependent network, the link weight of excitatory and inhibitory links are set to be constant. Increasing the link weight can lengthen the time that the network remains active. 


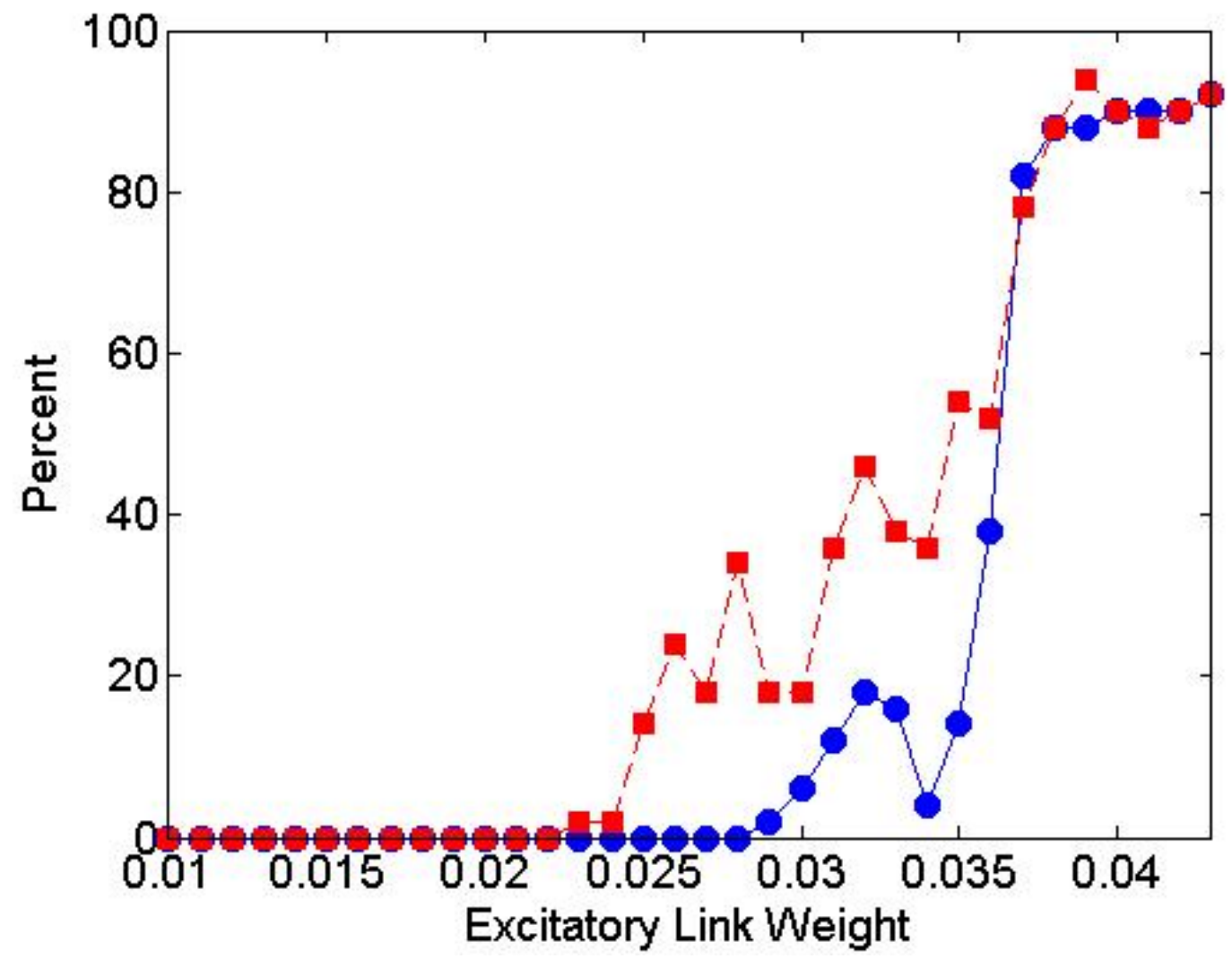

Figure 3.6. The probability of extended activity on a network is increased as the weight of the excitatory links is increased. The solid line represents the data of the distance dependent network. The dashed line shows values for the STDP network under the same conditions. Each point represents the fraction of 50 simulations that exhibits activity after 200 dimensionless time units. (Figure from reference [7].) 

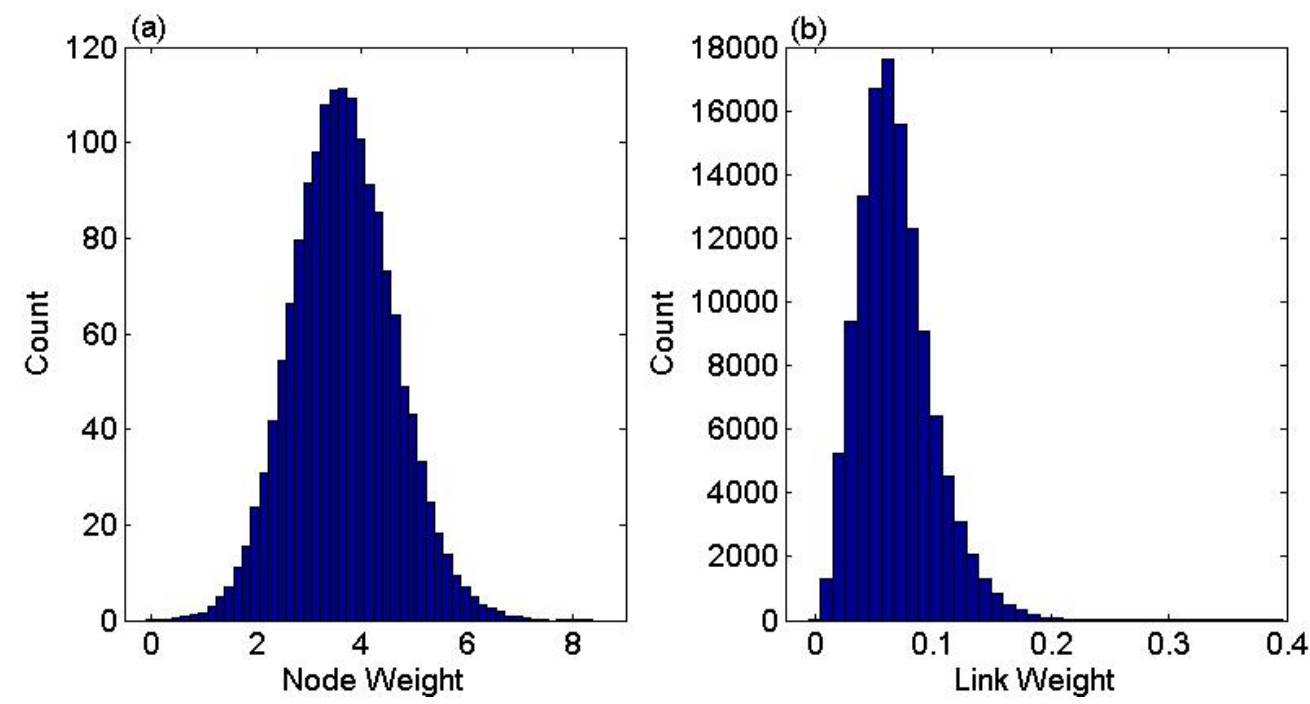

Figure 3.7. The stable distributions of node weights and excitatory link weights. The $Y$ axis represents the number of nodes and links. The distributions are based on the average result of $20 S T D P$ networks with the same parameters but different probability seeds. The network has 1600 nodes and 150,000 links. The initial excitatory link weight is 0.06 , and the (constant) inhibitory link weight is -0.08 . (Figure from reference [7].) 
For the STDP networks, we use the same initial excitatory link weights and the same initial inhibitory link wights. The link weights change based on the activity of the corresponding cells. If the STDP network exhibits sustained activity, a stable distribution of link weights is observed. 


\section{Reference}

[1] A. J. Steele, M. Tinsley, and K. Showalter, "Spatiotemporal dynamics of networks of excitable nodes," Chaos 16, 015110-1-9 (2006).

Online Version 33, 34, 36, 38

[2] M. Tinsley, J. Cui, F. Chirila, A. Taylor, S. Zhong, and K. Showalter, "Spatiotemporal networks in addressable excitable media," Phys. Rev. Lett. 95, 038306-1-4 (2005).

Online Version 34

[3] H. Berry and O. Temam, "Characterizing self-developing biological neural networks: A first step towards their application to computing systems," in "Lecture Notes in Computer Science," volume 3512, 306-317 (2005).

Online Version 37, 38

[4] H. K. D. H. Bhadeshia, "Neural networks in materials science," ISIJ International (1992). Online Version 39

[5] M. C. W. Rossum, G. Q. Bi, and G. G. Turrigiano, "Stable hebbian learning from spike timing-dependent plasticity," J. Neurosci. 20, 8812-8821 (2000).

Online Version 40

[6] C. Zhou, L. Zemanova, G. Zamora, C. C. Hilgetag, and J. Kurths, "Hierarchical organization unveiled by functional connectivity in complex brain networks," Phys. Rev. Lett. 97, 238103-1-4 (2006).

Online Version 40 
[7] A. J. Steele, Collective Behavior in Chemical Systems, Ph.D. thesis, West Virginia University (2007). 44, 45, 46 


\section{Chapter 4}

\section{A Chemical Neuronal Network: Simulations and Experiments}

\subsection{Experimental System}

\subsubsection{Chemical System}

Stock solutions are prepared using analytical grade chemicals and deionized water and are refrigerated before use. The concentrations of the reactants are $\left[\mathrm{BrO}_{3}^{-}\right]=0.458 \mathrm{M}$, $\left[\mathrm{CH}_{2}(\mathrm{COOH})_{2}\right]=0.0125 \mathrm{M},\left[\mathrm{BrCH}(\mathrm{COOH})_{2}\right]=0.188 \mathrm{M}$, and $\left[\mathrm{H}_{2} \mathrm{SO}_{4}\right]=0.512 \mathrm{M}$. The catalyst concentration in the silica gel is $\left[\mathrm{Ru}(\mathrm{bpy})_{3}^{2+}\right]=2.2 \mathrm{mM}$. Ruthenium bipyridil stock solution is prepared by dissolving ruthenium bipyridil sulfate in deionized water. The silica gel, which is used as a medium to immobilize the catalyst ruthium(II), is made of waterglass ( $15 \%$, weight ratio of sodium silicate to deionized water), ruthium(II) solution, and $\mathrm{H}_{2} \mathrm{SO}_{4}$, which used as hardening agent.

The thickness of gel is $0.3 \mathrm{~mm}$, and the working area of $25 \mathrm{~mm} \times 30 \mathrm{~mm}$ is divided into 
a $12 \times 10$ array of cells by imposing an appropriate pattern of light intensity. During the experiment, the thin silica gel is bathed in continuously refreshed solution and the system is maintained in an excitable steady state.

\subsubsection{Chemical Waves in the BZ Reaction}

Propagating waves can be observed when the BZ reaction is carried out using an immobilized catalyst. In the ruthenium(II) catalyzed BZ reaction, the wave front includes an increased concentration of $\mathrm{HBrO}_{2}$. Following the wave front is the oxidized catalyst, $\mathrm{Ru}(\mathrm{bpy})_{3}^{3+}$, which is gradually reduced in the tail of the wave [1].

The BZ reaction waves arise from a combination of diffusion and reaction $[2,3]$. In the excitable medium, waves are initiated by a perturbation. In the light sensitive BZ reaction, a small dark area, dust, or a silver needle touching the surface of the gel can serve as a wave initiator. After the perturbation, the initial wave can be visualized as a bright point, which then propagates by the diffusion of the autocatalyst bromous acid. The wave typically spreads through the medium with a characteristic velocity [2].

If we increase the light intensity on the excitable medium, the excitability of the region is decreased and the visible waves decay until they can not be seen. Now the state of the medium is defined as subexcitable. 


\subsubsection{The Instrumental Setup}

Figure 4.1 shows a schematic representation of the experimental setup. Different regions of the gel can be illuminated independently by a projector according to a computed illumination pattern. A bandpass filter is used to select the light at $440 \sim 460 \mathrm{~nm}$ corresponding to the absorption band of ruthenium(II). The beam splitter reflects the light onto the gel, while allowing observation with a video camera. The lens of the projector is adjusted in order to focus on a small area, e.g. $3.0 \mathrm{~cm} \times 3.0 \mathrm{~cm}$. A CCD video camera sends images of the spatiotemporal behavior to the computer for calculation of the real-time feedback. The reactor vessel is placed into a temperature-controlled base and the temperature is maintained at $5^{\circ} \mathrm{C}$.

\subsubsection{Design of the Illumination Pattern}

Figure 4.2 shows the design of the illumination pattern in our experiment. In panel (a), the large yellow rectangle represents a slab of gel. The dark, gray and white areas show the different light intensities from the projector. The light intensity of dark square is zero, which is used as a wave source by maintaining the medium in the excitable regime. The white area is the area of the highest light intensity, and it is used as barrier to stop wave propagation. The gray area on the right-hand side is the network area, where the array of cells is defined.

Figure 4.2(b) shows the process to initiate the network. A wave is initiated in the dark region, which enters into the network area. We decrease the background light intensity of the network area to zero for $3 \mathrm{~s}$ in order to allow wave propagation into the 3 neighboring 


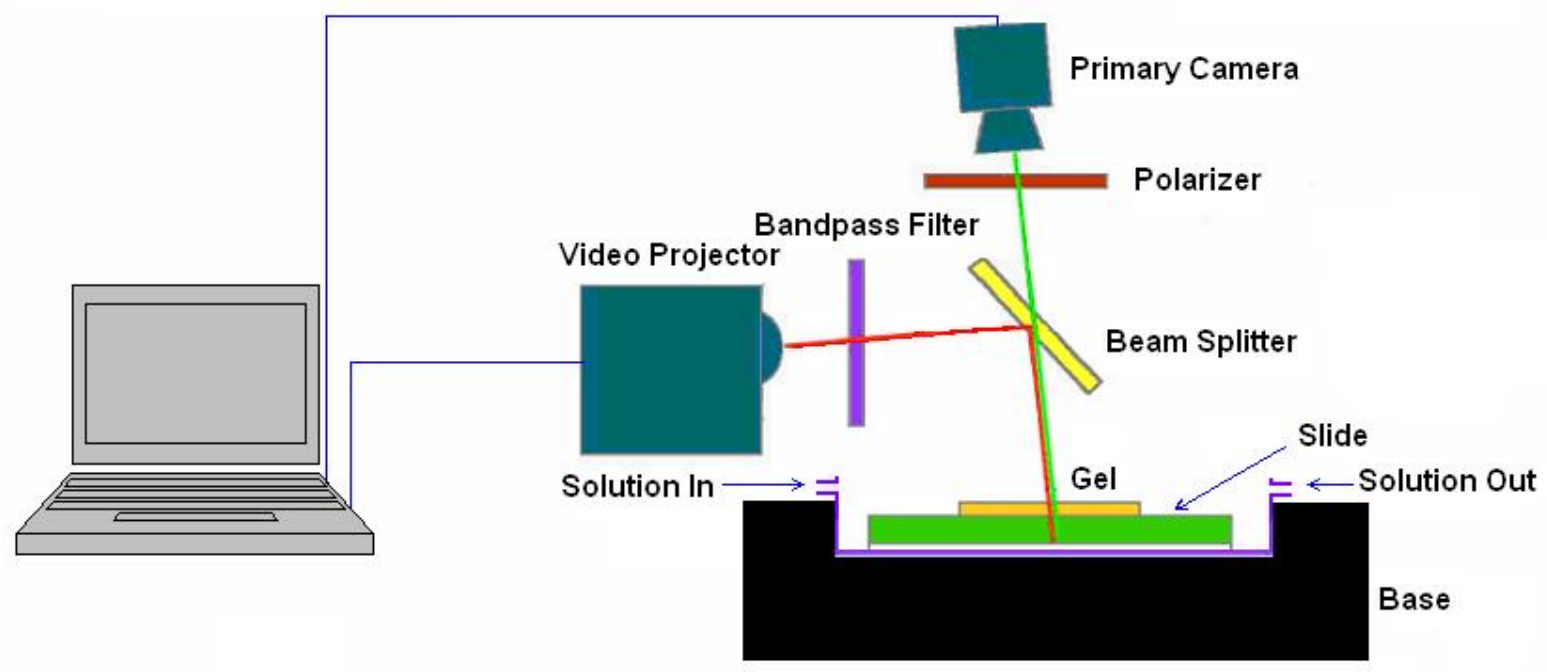

Figure 4.1. The instrumental setup for the experiment. The reaction occurs in a thin silica gel in which the catalyst ruthenium(II) is fixed. The gel is cast onto a glass slide and is bathed in the flowing reactant solution. 


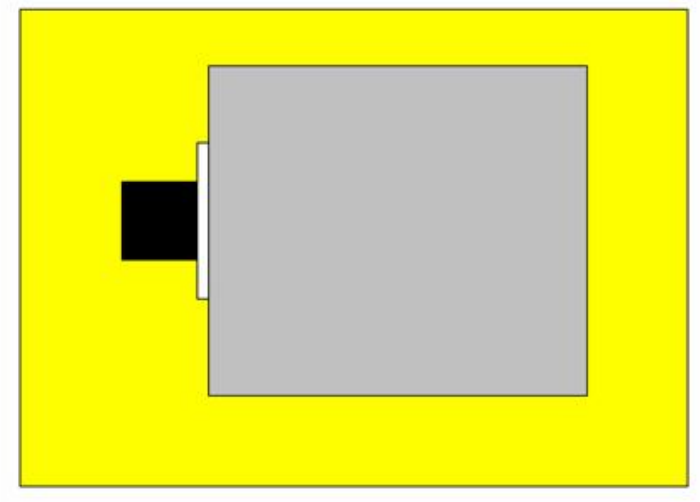

(a)

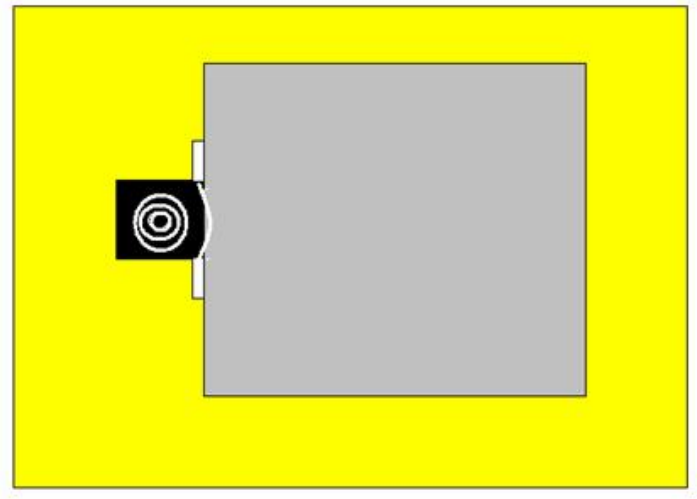

(b)

Figure 4.2. (a) The illumination pattern imposed onto the gel. (b) Initialization of the network with wave activity.

cells. The light intensity is then returned to the background value and images are taken every $1.0 \mathrm{~s}$ by the camera to record the activity of the network.

\subsection{Simulation Results}

In the simulation, we use the distance dependent network model to control the network. The weights of the excitatory links are set to 0.04 and the inhibitory link weight is -0.08 . The simulations show that the activity of the system is maintained longer as the number of links is increased. We are interested in the activity of the network when the link number is close to the threshold. The network consists of $20 \times 20$ nodes and each node includes $15 \times 15$ pixels in the simulations. Figure 4.3 shows the simulated activity of this network 
when the number of links is equal to 700 , and Fig. 4.4 shows the simulated activity when the number of links is equal to 800 .

Comparing the two simulation results, we find that when the number of links is equal to 700, after initiation of the center nodes, the network nodes connected to the center nodes by the links become excited, which can cause the whole network to become active. But the activity only lasts for a certain time and eventually ceases. However, when the number of links is increased to 800, after initiation of the same center nodes, the network activity lasts indefinitely. Figure 4.5 shows the two different regimes of the network activity. Panel (a) shows the process from initiation to finish for the network with 700 links, and panel (b) shows the continuing activity for the network with 800 links.

\subsection{Experimental Results}

In our experiment, the network consists of $12 \times 10$ nodes and each node includes $43 \times 40$ pixels. Applying the distance dependent network model, we fix the weight of links such that the excitatory link weight is equal to 0.04 and the inhibitory link weight is -0.08 . We find that when the number of links is greater than 400, the system can maintain a certain amount of activity for a long period of time, while if the number of links is lower than 400, the activity of the system will finally cease. The duration of the period of activity increases as the number of links is increased.

But during the experiment, we did not observe this kind of self-sustained activity when one center, two centers, four centers and even more centers of self-sustained static patterns were initiated in the experimental medium. Figure 4.6 (a) shows network behavior with one 

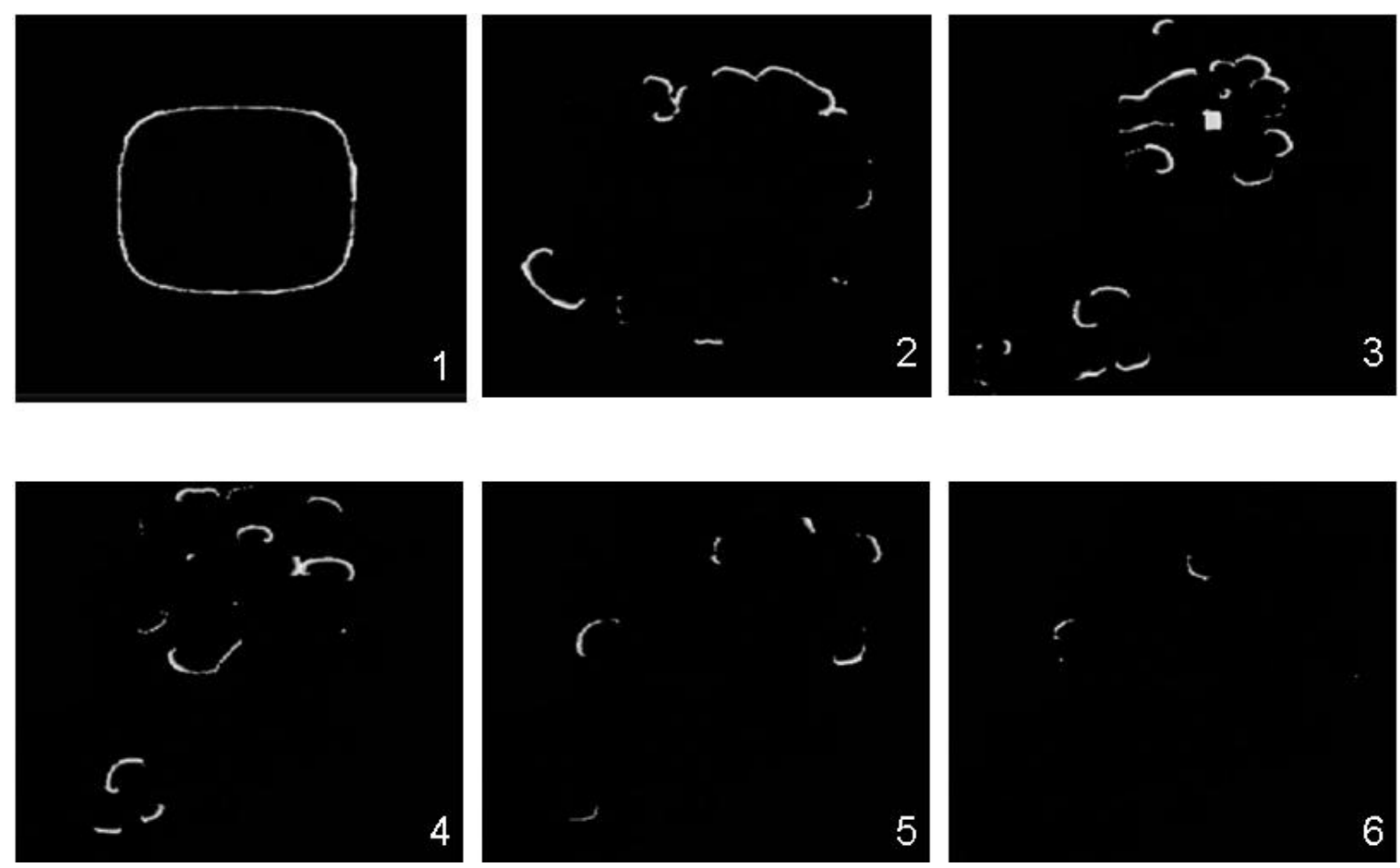

Figure 4.3. Simulation of the activity of the network after the center nodes were initiated, when the links number is equal to 700 . The network consists of $20 \times 20$ nodes and each node includes $15 \times 15$ pixels. 

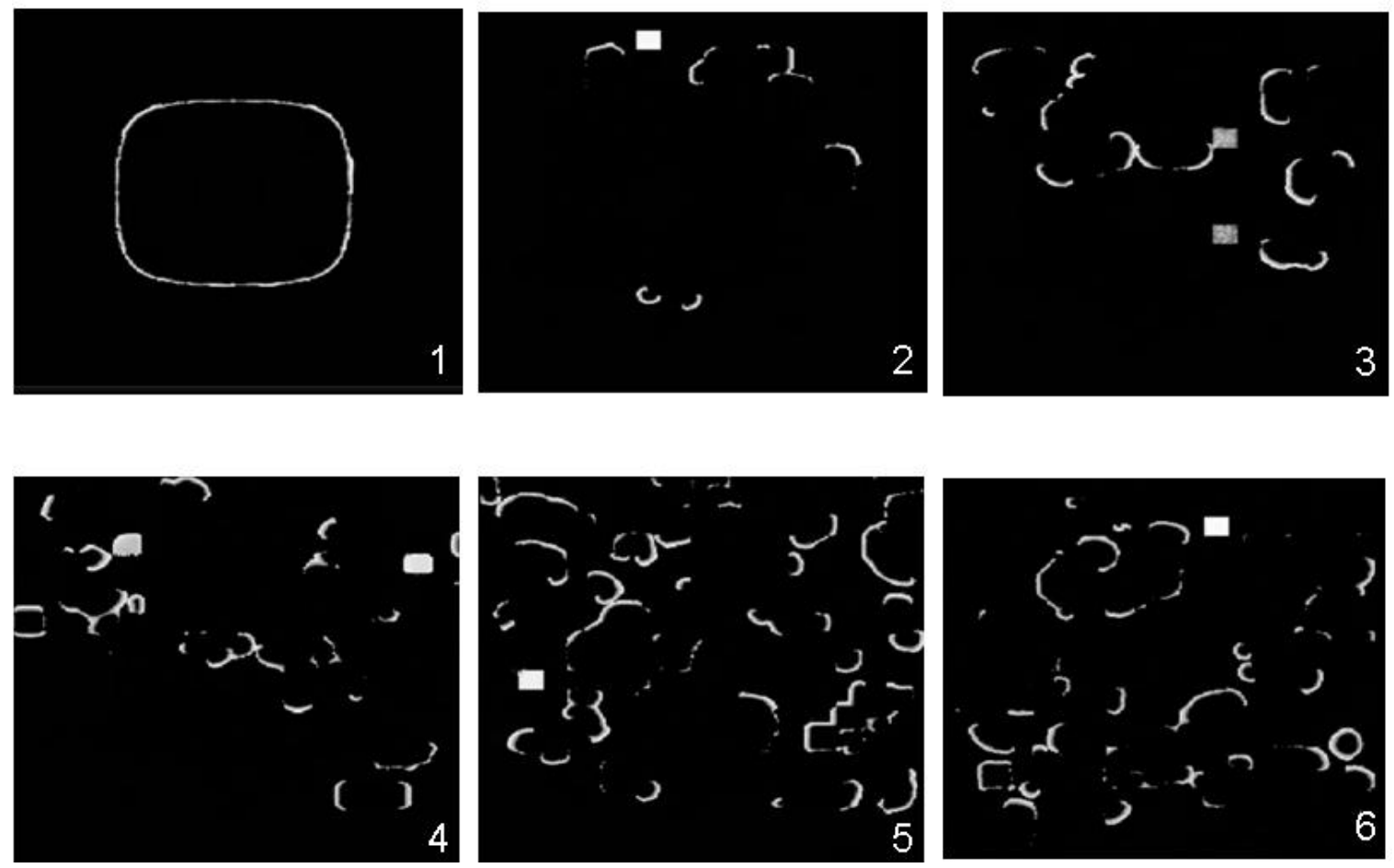

Figure 4.4. Simulation of the activity of the network after the center nodes were initiated, when the number of links is equal to 800 . The network consists of $20 \times 20$ nodes and each node includes $15 \times 15$ pixels. 

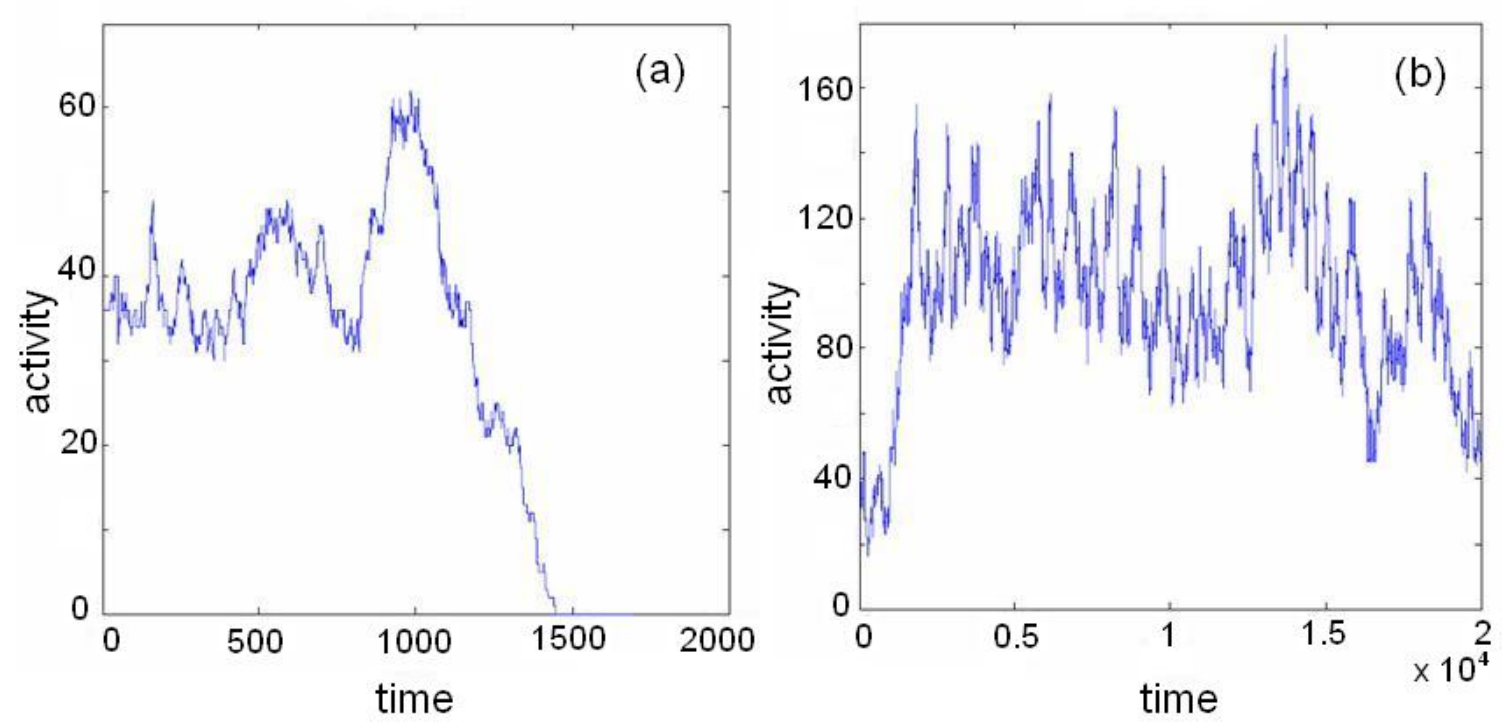

Figure 4.5. Activity of networks with different number of links. Panel (a) shows the activity of the network when the number of links is equal to 700. Panel (b) shows the activity of the network when the number of links is 800 . The y axis represents the number of the active nodes, and the $\mathrm{x}$ axis represents dimensionless time. 
center, and the number of links equal to 300. Figure 4.6 (b) shows network behavior with two centers, and the number of links equal to 350. Figure 4.7 (c) shows four centers with 300 links and Fig. 4.7 (d) shows more centers with 400 links.

Comparing these four groups of images, we see that the centers of self-sustained static patterns easily form in the experimental medium. The number of links determines the duration of activity. When the number is over 400, the activity dramatically increases and the network can remain active without limit. But in the real world, we do not see such self-sustained activity in networks. For example, in neuronal networks, the neurons do not continually auto-excite after receiving one stimulation; on the contrary, fatigue appears in the neuronal network after certain period of activity [4]. We look for the cause of these selfsustained static patterns and analyze the patterns in detail, finding the cells in the active centers and all the links related to them.

From the analysis of Fig. 4.8, Fig. 4.9 and Fig. 4.10, we see that the self-sustained static patterns are formed based on the existence of an active loop. Figure 4.8 (a) is a picture of a static pattern with two centers, Fig. 4.8 (b) is the corresponding light intensity, and Fig. 4.8 (c) is a schematic diagram that shows the relationship between the two center cells $(24$ and 33 cell) and their linked cells by excitatory or inhibitory links. The figure shows that for cell 24, there exists six input excitatory links from cells $5,33,35,15,33,35$, in which two links from cell 33 are active, and one inhibitory link from cell 35 . While for cell 33, there are five excitatory links from cells 22, 25, 24, 28, in which two links from 24 are active. And because cell 24 and cell 33 are neighbors, a diffusive link exists between them. Therefore, two active loops with excitatory links and one diffusive loop are formed.

We now analyze the image of the self-sustained static pattern with one center (Fig. 4.9 (a)). 

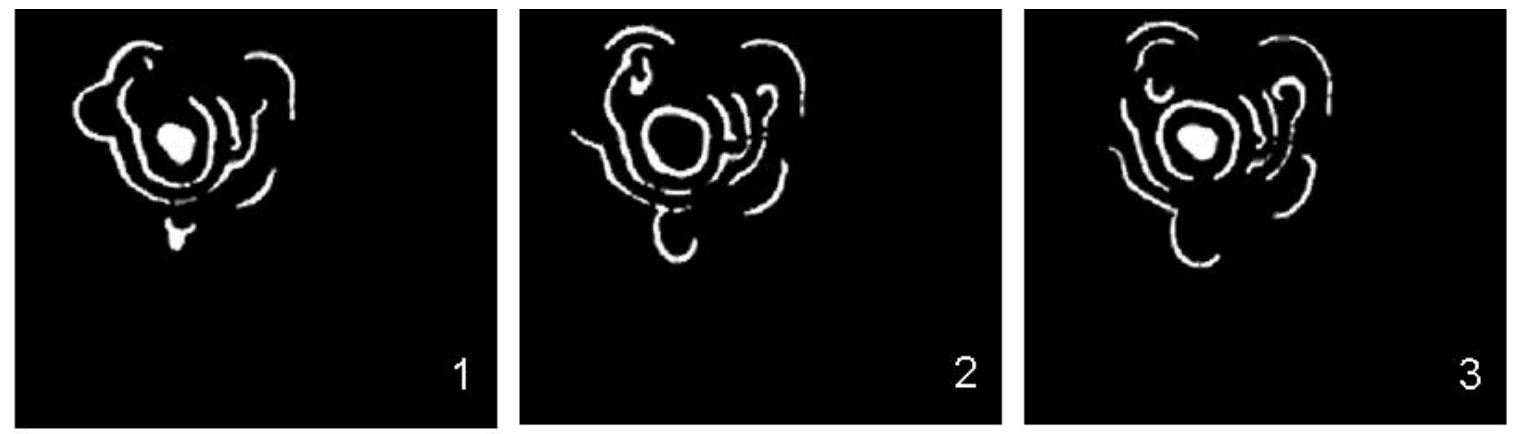

(a)
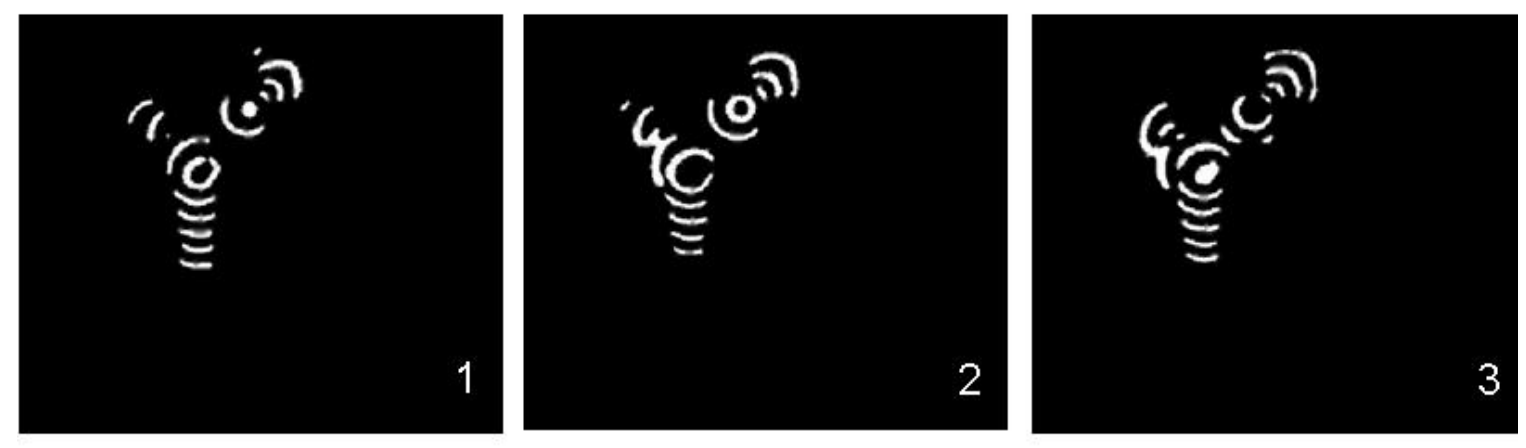

(b)

Figure 4.6. Self-sustained static patterns with one and two centers. Images (a) show the activity in a self-sustained pattern with one center. The number of links is equal to 300. Images (b) show a self-sustained static pattern with two centers, and the number links is equal to 350 . 

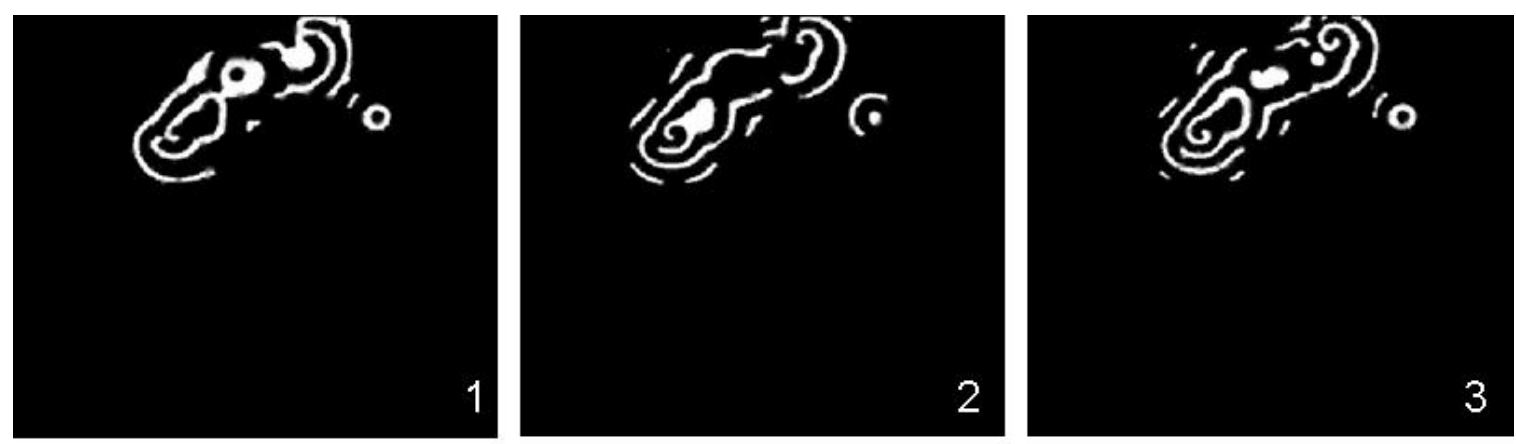

(c)
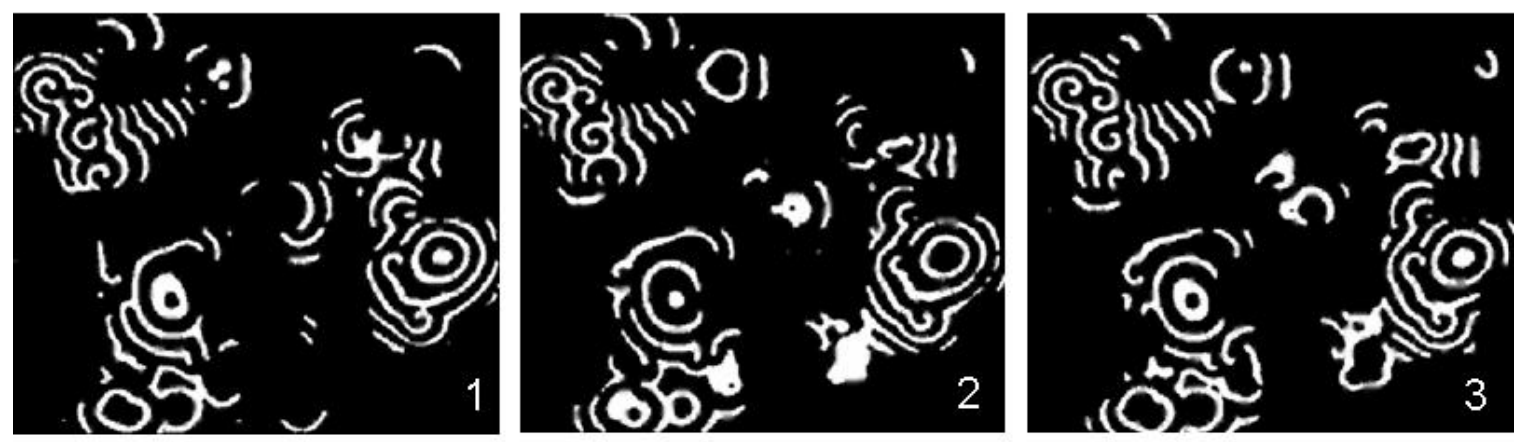

(d)

Figure 4.7. Self-sustained static patterns with four and more centers. Images (c) show a self-sustained static pattern with four centers, and the number of links is equal to 300 . Images (d) show a self-sustained static pattern with more centers, and the number of links is equal to 400 . 


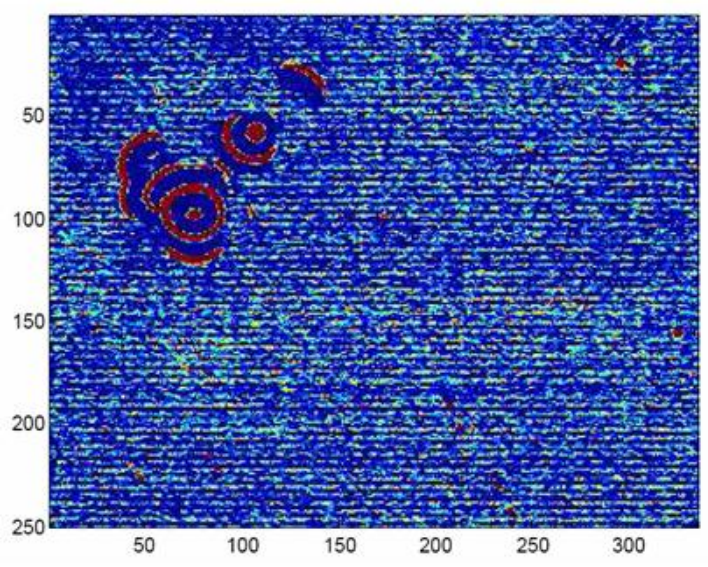

(a)

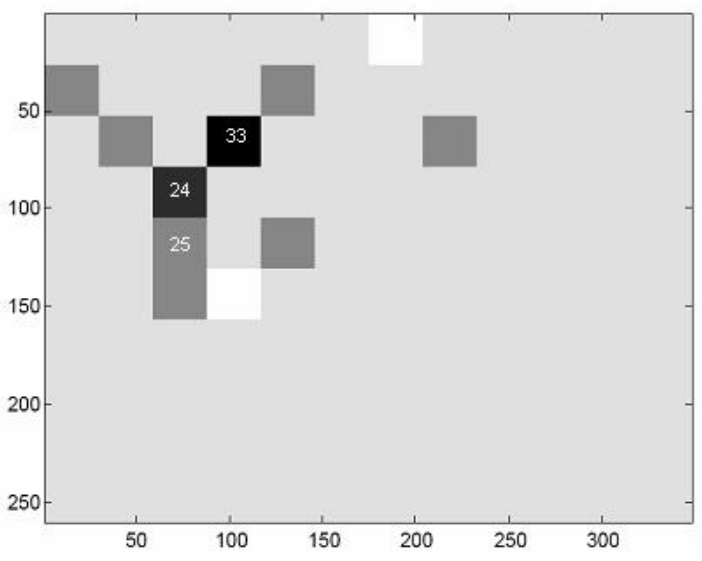

(b)

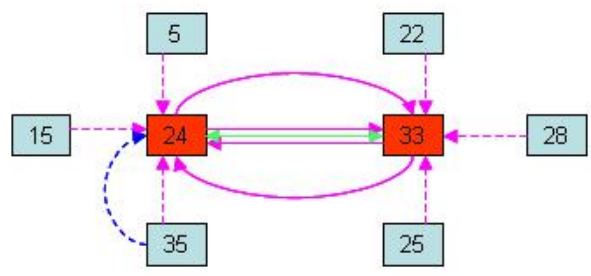

(c)

Figure 4.8. (a) A color map of the two centers of the self-sustained static pattern. (b) The light intensity arrangement of the cells on the experimental medium corresponding to (a). The numbers represent the corresponding cells at the active centers. In this figure, number 24 and number 33 are the two active center cells of the network. (c) The link assignments of these two cells. Here, the purple arrows represent the excitatory links and the blue arrows represent the inhibitory links. The green arrow represents a diffusive link. The solid lines represent the active links, while the dashed lines represent the inactive links. Between cells 24 and 33, there are two active loops based on active excitatory links and one diffusive loop. 


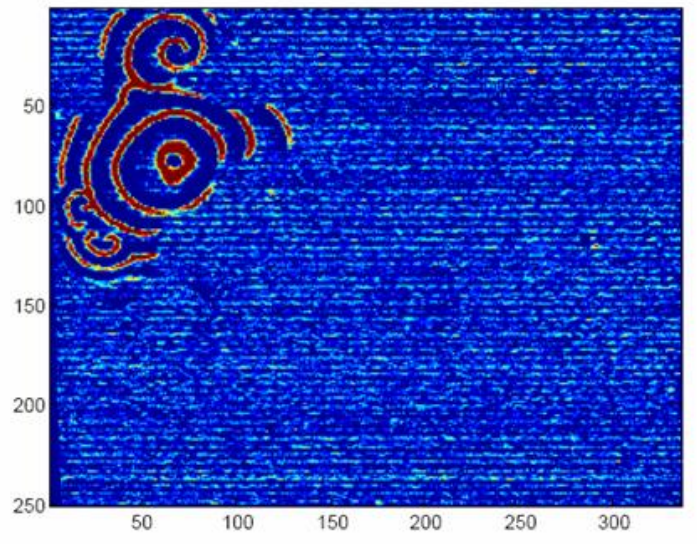

(a)

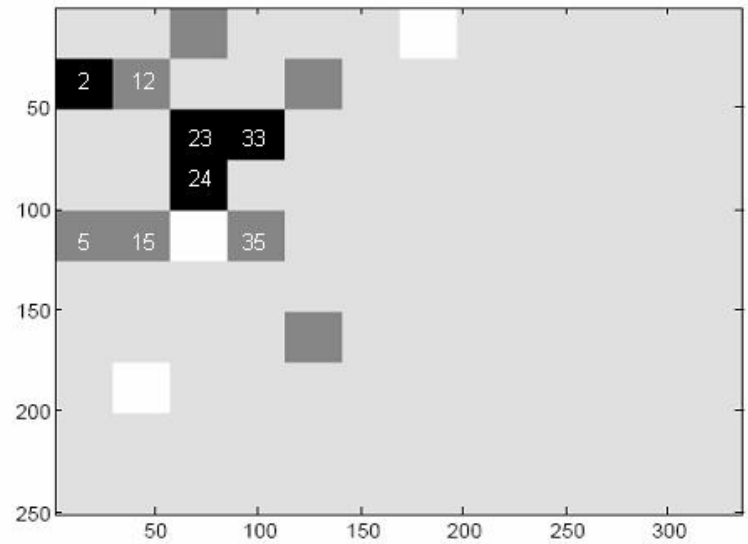

(b)

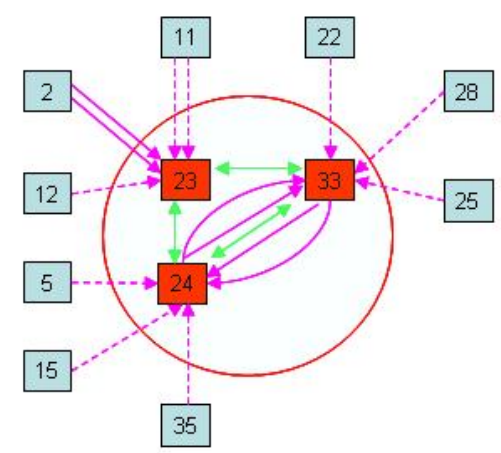

(c)

Figure 4.9. (a) A color map of one center of the self-sustained static pattern. (b) The light intensity arrangement of the cells at the corresponding time. In this figure, cells 2, 23, 24 and 33 are the active cells of the network, while cells 23, 24 and 33 are the active centers. (c) The link assignment of these three center cells. For each active center cell, there exists two input active excitatory links and two input diffusive links, and active loops are formed between cells 24 and 33 by four active excitatory links. The arrow color has the same meaning as in Fig. 4.8. 


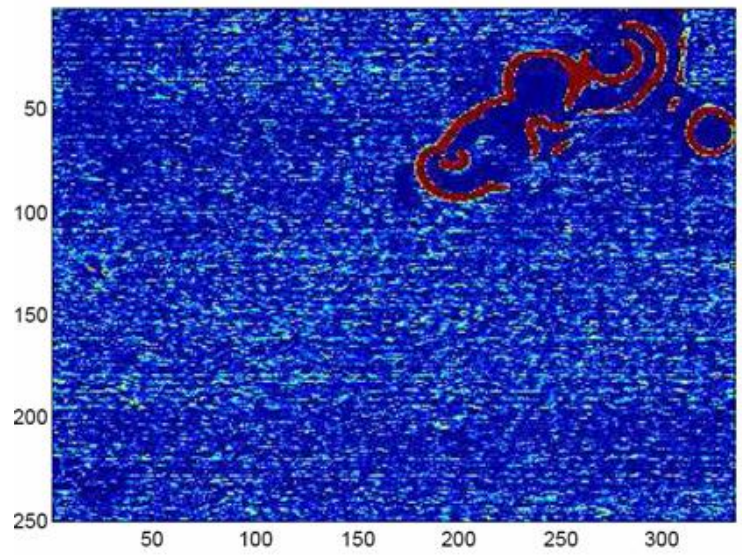

(a)

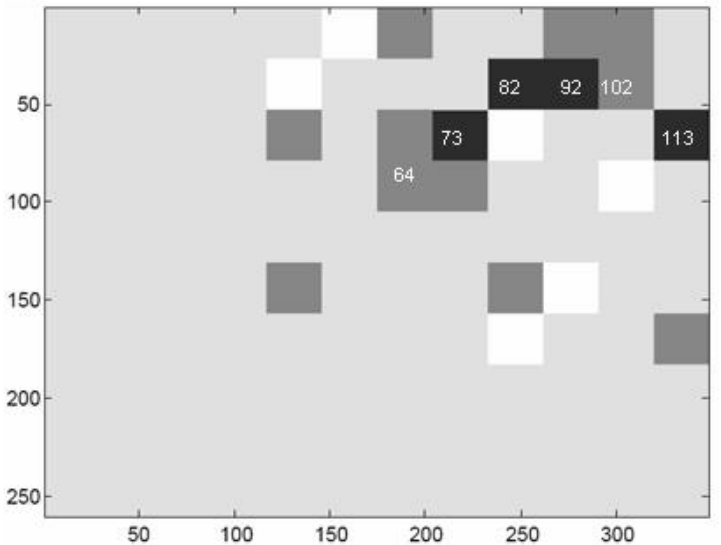

(b)

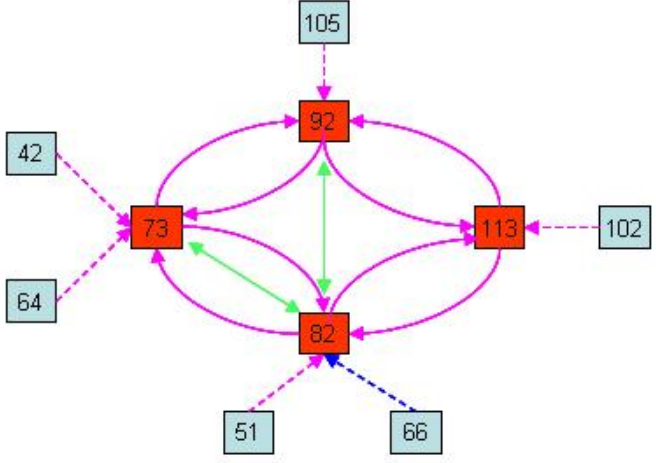

(c)

Figure 4.10. (a) A color map of the four centers of the self-sustained static pattern. (b) The light intensity arrangement of the cells at the corresponding time. In this figure, cells 73, 82, 92 and 113 are the active cells of the network. (c) The link assignments of the four center cells. Active loops are formed among these four active cells by the active excitatory links. There exists two diffusive loops between cell 73 and cell 82 and also cell 82 and cell 92. The arrow color has the same meaning as in Fig. 4.8. 
In Fig. 4.9 (b) we found that there are four active cells, and three of them (23, 24 and 33) are in the active center. Because these three cells are close neighbors, their combined behavior resembles a one center static pattern. The bright excitation center is larger than other centers in the patterns with more centers. Figure 4.9 (c) shows the interactions among these three active cells and their linked cells. For the cell 23, there exists five excitatory links from cells 2, 12, 11 and two from cell 2 are active. For the cell 24, there are six excitatory links from cells 5, 33, 35, 15, and two links from 33 are active. Cell number 33 has five excitatory links from cells 22, 25, 24, 28 and two from 24 are active. Cells 23, 24 and 33 are neighbors with the diffusive links. Between active cells 24 and 33, there are two active loops that originate from active excitatory links, and one diffusive loop that originates from a diffusive link. Cell 23 is also excited by the two active excitatory links from cell 2 and two diffusive links from the neighbors 24 and 33. We have performed a similar analysis of the self-sustained static pattern with four centers .

From Fig. 4.10 (c) we find that the four active cells form active loops among them through the active excitatory links. If the number of the active loops is $\geq 2$ between two cells, the self-sustained active center may appear on these two cells, and the diffusive loops between them can also increase this self-sustained activity. If the number of links increases, the probability of loops that can generate self-sustained active centers becomes greater.

In order to match the simulated behavior representing the neuronal network, we modify our chemical model to eliminate self-sustained static patterns. We add a rule that if a cell is continually excited for a certain period of time, the system will automatically increase the light intensity of the cell to eliminate the activity. With this modification, the static selfsustained activity was suppressed. Figure 4.11 and Fig. 4.12 are images of the experimental 

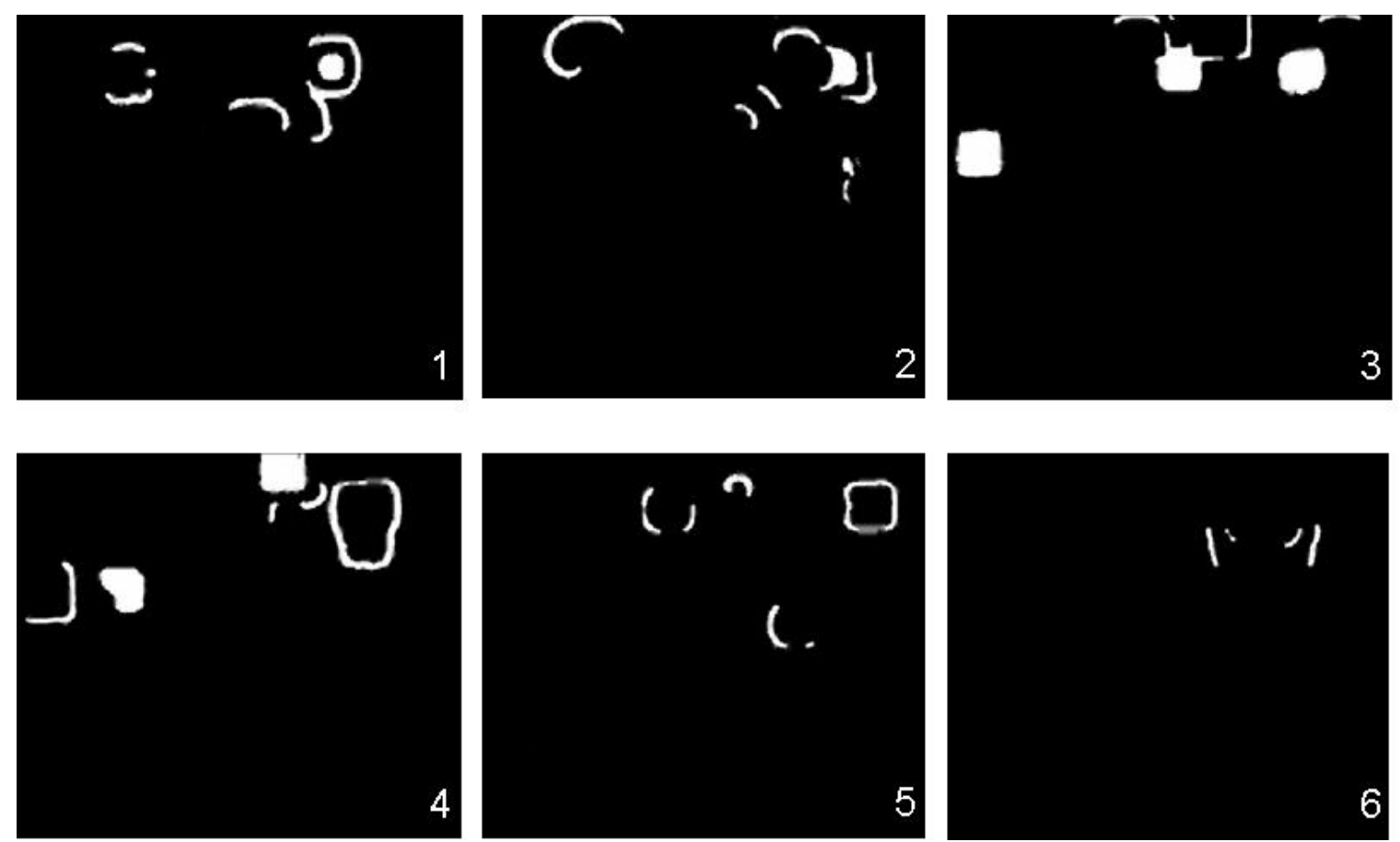

Figure 4.11. Limited restricted unsteady pattern. The figure shows snapshots of the network with the number of links equal to 400 .

results with the modified model. Figure 4.11 is the network behavior with 400 links, while Fig. 4.12 is the behavior of the network with 500 links. From these results, we see that 500 is the threshold value for the new network. If the number of links is equal to or greater than this value, the network activity is long lived.

In the experiments, we use a camera to record the activity of the network. We count the number of active cells in each image. The average number over the course of the experiment gives the average activity of the system. This analysis can also be used in the simulations. We accumulate the number of active nodes at each time unit, and then divide by total time. We therefore obtain the average activity in the simulated neuronal network. Figure 4.13 shows the average activity of the network for different number of links. Panel 

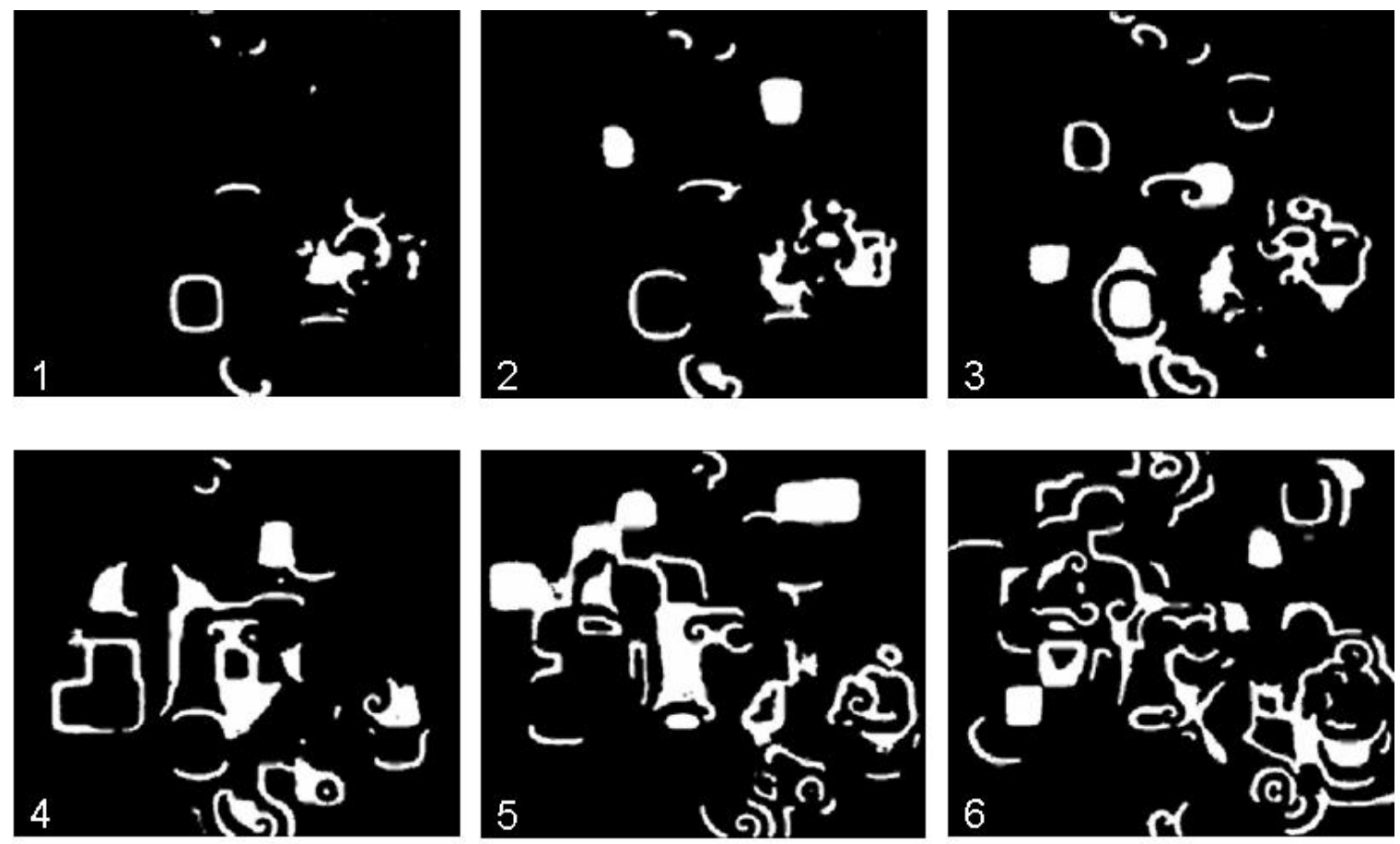

Figure 4.12. Restricted unsteady pattern. The figure shows snapshots of the network with the number of links equal to 500 . 

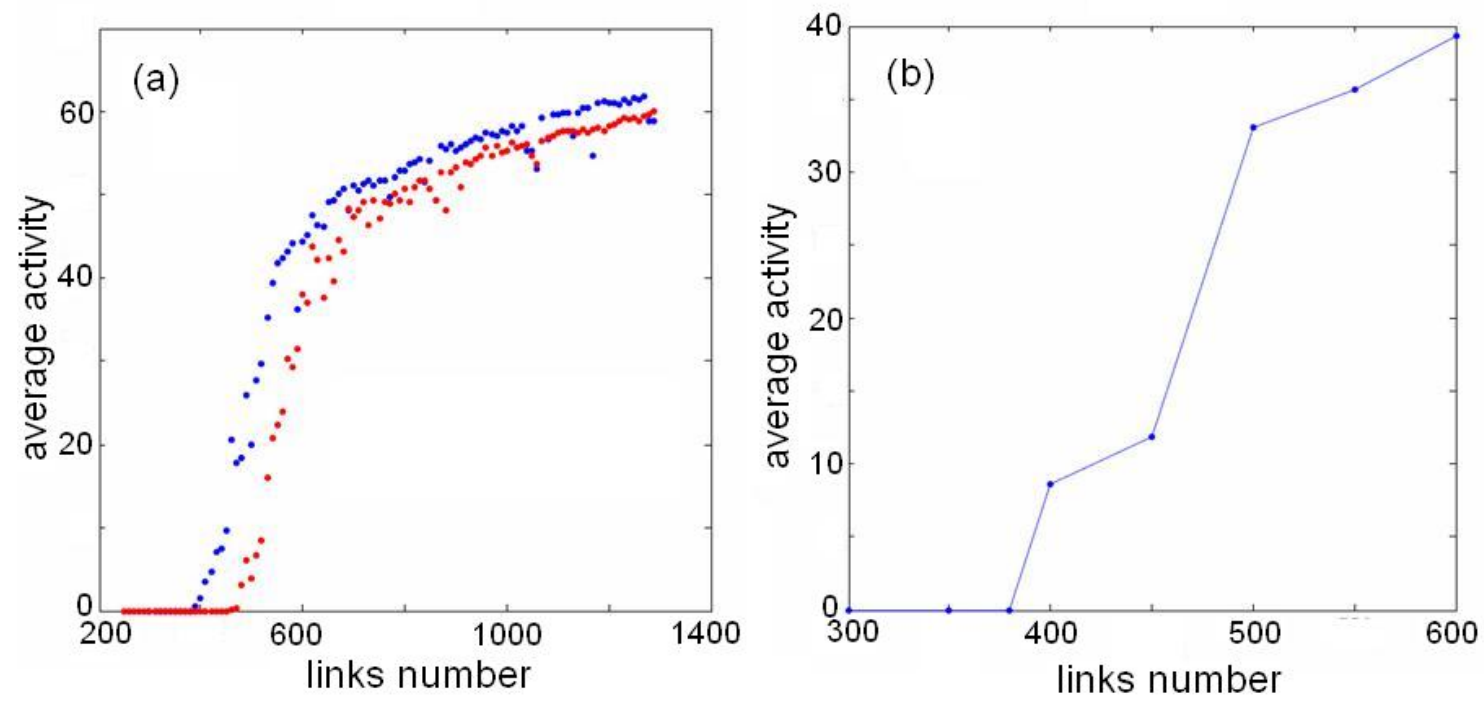

Figure 4.13. The simulated and experimental average activity versus the number of links. Panel (a) shows average activity for two sets of simulations. The model is a $10 \times 10$ array of cells, with each cell having $40 \times 40$ pixels (blue) or $60 \times 60$ pixels (red). We see that the larger the size of the cells, the lower the average activity for the same number of links in a network. Panel (b) shows the average activities in the experiment. The working area consists of $12 \times 10$ cells.

(a) is the numerical simulation and panel (b) is the experimental results. Comparing these two figures, we see that there exist a threshold number of links at which the activity of the network sharply increases, both in the simulated and in the experimental system. 


\subsection{Summary}

A two dimensional network with excitable nodes has been implemented in experiments and numerical simulations. In the simulations, we obtained the network behavior with different number of links and an analysis of the activity. Through the average activity measurements, we determined the threshold number of links, which determines the duration of activity in the network system.

In a comparison with the numerical simulations, the experimental system shows some special features. When the link assignment is based only on the distance dependent rule, which we call the free link model, self-sustained static patterns are found. Such static patterns are not observed in simulations. These patterns deviate from the behavior of actual neuronal networks. We therefore analyzed the detailed structures in the experimental system and found that active loops are responsible for the formation of self-sustained static patterns. After restricting the assignment of links based on the free link model in order to avoid such loops, we have successfully eliminated the static patterns. We use the average activity to quantitatively describe the activity of different states of the network. In a same sized network, the threshold number of links in this model is higher than that in the free link model, and the behavior is similar to the behavior in the simulations.

We have also applied the STDP model to the experimental system; however, we were unable to observe any obvious differences when comparing it to the restricted pattern system, since the threshold number of links remained around 500 . 


\section{Reference}

[1] R. Field and M. Burger, Oscillations and traveling waves in chemical systems (John Wiley \& Sons, Princeton, NJ, 1985). 51

[2] A. Winfree, The geometry of biological time (Springer-Verlag, New York, 2000). 51

[3] E. J. Reusser and R. J. Field, "The transition from phase waves to trigger waves in a model of the Zhabotinskii reaction," J. Am. Chem. Soc. 101, 1063-1071 (1979). Online Version 51

[4] J. Kanga, B. Choia, H. Leea, J. Kimb, and K. Kimb, "Neural network application in fatigue damage analysis under multiaxial random loadings," International Journal of Fatigue 28, 132-140 (2006).

Online Version 59 


\section{Chapter 5}

\section{Dynamical Quorum Sensing and Kuramoto}

\section{Transitions to Synchronization}

\subsection{Abstract}

Many oscillatory systems exist in nature, such as the flashing of fireflies [1], the metabolism of yeast cells [2], the physiological rhythm of heart cells [3], and the periodic firing of neuron cells. Investigations have characterized the individual oscillating mechanisms of these systems and discovered that they can form collective behavior due to the interactions between them. The simplest collective behavior of oscillators is phase or frequency synchronization. The flashing of a group of fireflies and the rhythmic activity of a heartbeat are examples of timekeeping behaviors that arise from the coupling of oscillators.

A. T. Winfree [4] and Y. Kuramoto [5] carried out pioneering research on synchronization in biological systems. In the 1980s, Kuramoto first described the mechanism of synchronization of a large number of phase oscillators in a global coupling mean field. In his description, 
the oscillators' transition from random phases to synchronization is a sigmoid growth in their collective amplitude. The collective signal of the oscillators is dependent on the coupling strength. When the coupling strength is lower than a critical threshold, the oscillators are phase randomized and the collective signal is flat and noisy. If the coupling strength is above this critical threshold value, a collective rhythm appears and the amplitude of the rhythm rises sigmoidally as the coupling strength increases. This transition of oscillators from random behavior to synchronization is referred to as Kuramoto synchronization. This phenomenon was first reported in 1974 in the suspensions of cells of the slime mold Dictyostelium discoideum [6]. In 2002, J. L. Hudson's group experimentally studied Kuramoto synchronization in global coupling of electrochemical oscillators [7].

Thirty years ago, Aldridge and Pye [8] discovered another distinct type of densitydependent transition to synchronization in suspensions of yeast cells. In recent years, experimental studies have revealed that this type of transition is a quorum-sensing transition. When the cell density is lower than a critical value, the collective system of cells is quiescent rather than unsynchronized. If the cell density is over the critical value, the system is synchronously oscillating [9]. Many transitions found in nature are quorum-sensing transitions, such as bioluminescence in Vibrio fischeri [10-12] and the formation of biofilm in Pseudomonas aeruginosa [13].

Figure 5.1 is a schematic diagram that shows the two different transitions to synchronization. Figure 5.1(a) illustrates the quorum-sensing process. The individual oscillators are stable and have no oscillations at low density. But when the number density increases to a certain level, oscillations appear abruptly, as shown in panel 1. The global behavior of these oscillators is shown in panel 2. At first, the whole system is stable until the density is 
increased to the critical value. The synchronization then suddenly arises, and the amplitude of the oscillation increases as the density increases. The Kuramoto synchronization process is shown in Fig. 5.1(b). For this type of transition to synchronization, at low number density, the individual oscillators have the same frequency and amplitude but different phases. The global behavior of these oscillators is flat and noisy. As the density is increased, more and more oscillators switch to the same phase and the collective behavior of the oscillators gradually changes to synchronization. Eventually, the phase of all the oscillators becomes the same, and the whole system exhibits synchronized oscillations, as panels 3 and 4 show.

We have studied populations of relaxation oscillators in order to characterize the two type of transitions to synchronization as a function of the population density and coupling strength (exchange rate) of the oscillators with the surrounding solution. Through changing the density of oscillators and the chemical exchange rate, we observe the Kuramoto synchronized transition and the quorum-sensing transition. The variation of the chemical exchange rate is carried out by changing the stirring rate. We use images to record the oxidation states of the chemical oscillators so that their synchronization can be characterized. We also use numerical analysis to characterize the Kuramoto and quorum-sensing transitions. 

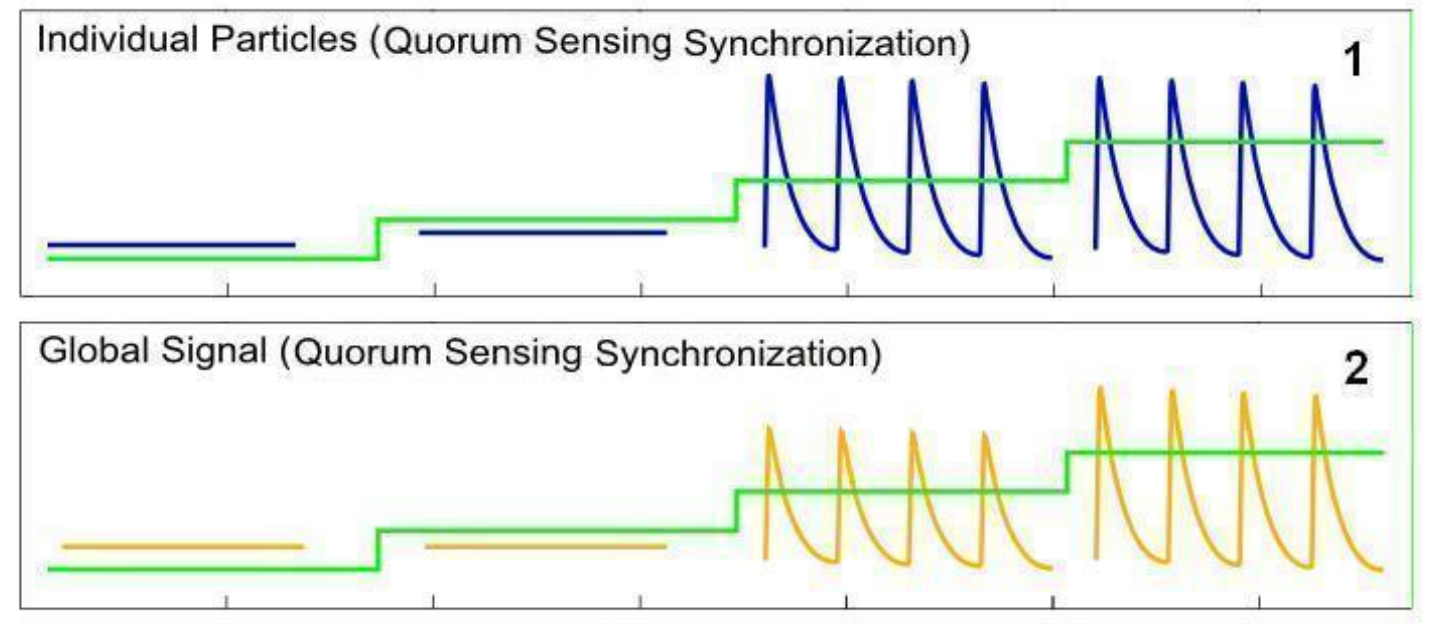

Time

(a)
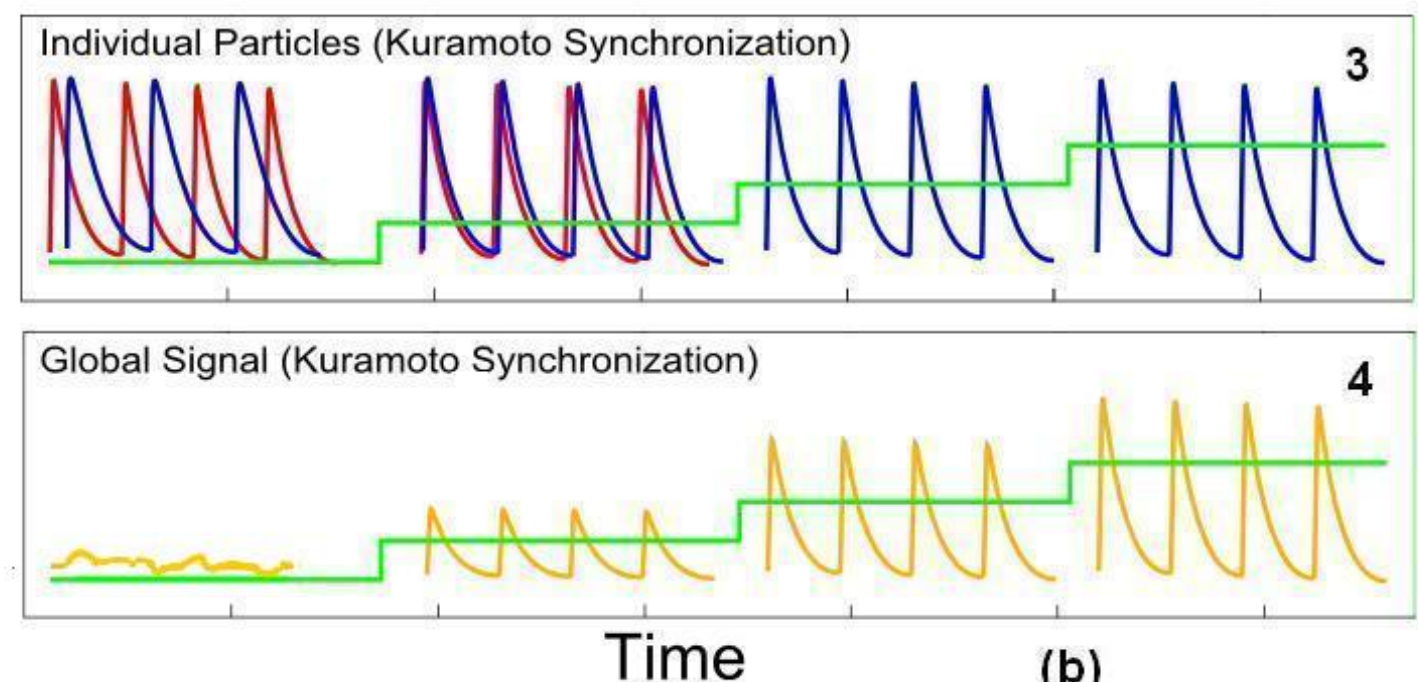

Figure 5.1. (a) Quorum sensing transition to synchronization. (b) Kuramoto synchronization. The brown lines represent the global behavior of the oscillators. The green lines represent the number density. The blue and red lines represent the different individual behaviors of the oscillators at different number densities. In panel 3, we see that the oscillating phases of the individual oscillators align as the oscillator density increases. 


\section{$5.2 \quad$ Experimental Setup}

\subsubsection{Catalyst Particle Preparation}

The chemical oscillators in our experiment are ferroin-coated microporous cation-exchange beads (DOWEX 50WX4-200). The beads, purchased from Fisher, are put into ferroin solution and stirred for 4 hours, allowing the ferroin catalyst to be loaded onto the beads. Ferroin, the common name for $\mathrm{Fe}(\mathrm{phen})_{3}^{2-}$, is the catalyst for the BZ reaction and is widely used as a redox indicator, whose color changes between red (the reduced form) and blue (oxidized form) during the reaction. The beads are then filtered and washed with distilled water and then $0.1 \mathrm{M}$ sulfuric acid. The catalyst-coated beads are left to dry at room temperature in air for 24 hours before being stored in a sealed bottle. The catalyst concentration on the beads is $1.7 \times 10^{-5} \mathrm{M}$.

An estimation of the number density of the beads in solution is given by the equation

$$
n=N / V_{s}=V_{p} / \bar{V} V_{s}
$$

where $N$ is the total number of beads in the solution. The volume displacement of wet beads $V_{p}$ is calculated by $V_{p}=5 V_{d}$, where $V_{d}$ is the volume of dry beads occupied by $20 \%$ of the volume displacement of wet beads. $V_{s}$ is the total volume of the solution. $\bar{V}$ is the average volume of each bead, which is calculated from the average diameter of beads $(\mathrm{d}=120 \mu \mathrm{m})$. For example, when a mass of $0.2 \mathrm{~g}$ of dry ferroin-loaded beads is added to $10 \mathrm{ml}$ of solution, the volume displacement of the wet beads is $0.17 \mathrm{ml}$, and therefore the total number of beads $N$ is $1.2 \times 10^{5}$ and the number density of the beads $n=1.2 \times 10^{4} \mathrm{ml}^{-1}$.

We analyzed the size of the beads and found they have a size distribution as shown in 
Fig. 5.2(a). Each bead loaded with catalyst has its own oscillatory period based on its size and the catalyst loading; therefore, we measure the oscillatory period distributions of these catalyst-loaded beads (see Fig. 5.2(b)). The oscillatory period of the beads was measured in unstirred BZ solutions, where the beads are arranged so there are no interactions between them. The concentrations of the reactants are $\left[\mathrm{NaBrO}_{3}\right]=0.49 \mathrm{M},[\mathrm{MA}]=0.14 \mathrm{M},\left[\mathrm{H}_{2} \mathrm{SO}_{4}\right]$ $=0.67 \mathrm{M}$, and $[\mathrm{NaBr}]=0.07 \mathrm{M}$. During the reaction, the color of the catalyst-loaded beads changes between red and blue depending on the state of the catalyst ferroin. The optical density of each bead can be recorded to track the oscillatory period of the bead. Figure $5.2(\mathrm{c})$ is an example of the time series of the optical density for two individual oscillating beads.

\subsubsection{Instrumental Setup}

The reactor is a cylindrical vessel made of glass and fitted with a flat viewing window on the side. A Pt redox microelectrode (MI-800 from Microelectrodes, Inc.) is used to record the chemical electropotential of the BZ solution. A digital camera (SPOT Insight IN1120, shutter speed $0.4 \mathrm{~ms}$ ) is used to photographically record the color change of the ferroin-loaded beads, and the viewing window is illuminated by two fiber-optic lamps. A magnetic stirrer (IKAMAG) is used to control the stirring speed of a $1.7 \mathrm{~cm}$ stirring bar. The stirring speed can be controlled from 100 to $900 \mathrm{rpm}$, and the beads are completely suspended in the BZ solution. We also have used a motor-driven impeller as a method to control the stirring speed and found that it has a higher exchange rate for a given stirring 


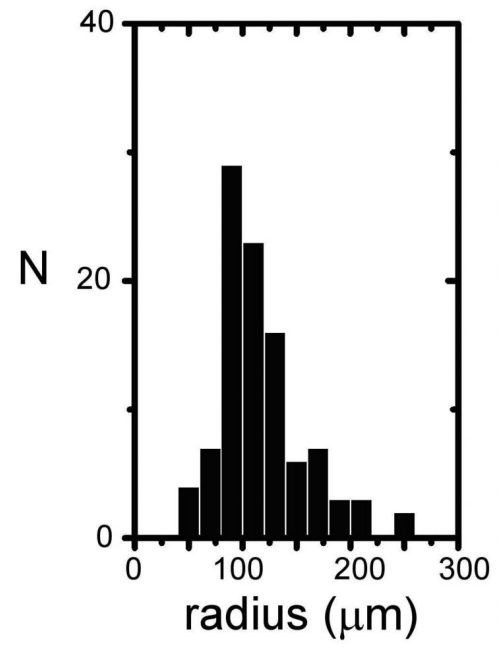

(a)

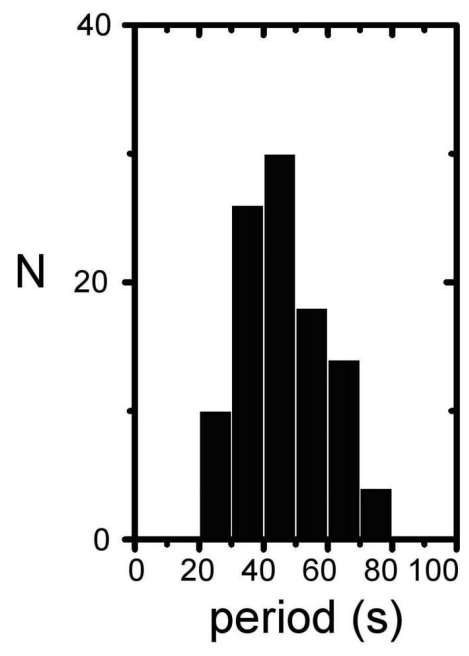

(b)

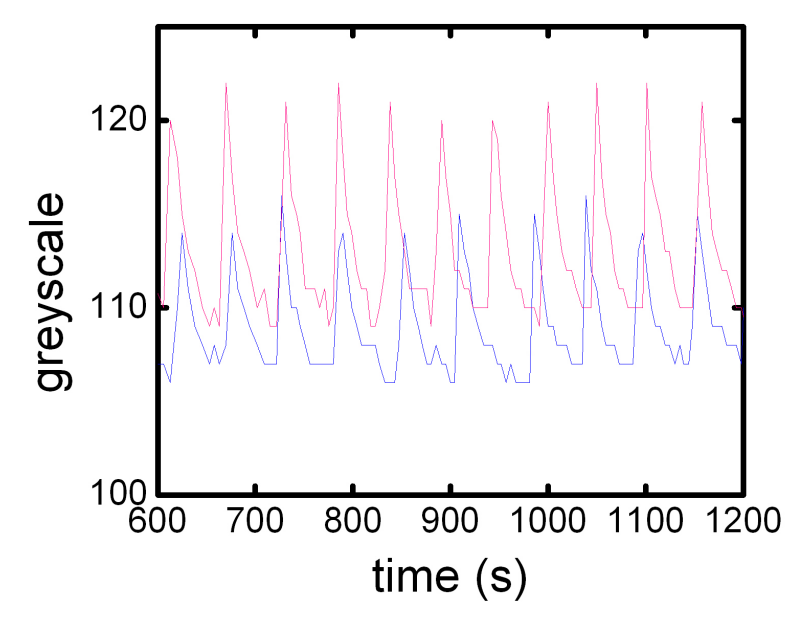

(c)

Figure 5.2. The bead radius and oscillatory period distributions in the unstirred BZ solution. (a) Radius distribution of beads (DOWEX 50WX4-200). (b)Oscillatory period distribution of beads (figure from Ref. [14]). (c) Time series in grayscale for two individual oscillatory beads. The concentrations of the solution: $\left[\mathrm{NaBrO}_{3}\right]=0.49 \mathrm{M},[\mathrm{MA}]=0.14 \mathrm{M}$, $\left[\mathrm{H}_{2} \mathrm{SO}_{4}\right]=0.67 \mathrm{M}$ and $[\mathrm{NaBr}]=0.07 \mathrm{M}$. 
speed than the magnetic stirrer. Our experimental results presented here are obtained by using a magnetic stirrer. Adding a known amount of dry catalyst-loaded beads into the stirred BZ solution increases the number density of the beads. The volume of the solution is $10 \mathrm{ml}$ and the solution temperature is $20^{\circ} \mathrm{C}$. Figure 5.3(a) and Fig. 5.3(b) show two images of the experimental setup, and Fig. 5.3(c) is a sketch of the experimental setup.

\subsubsection{The Optical Intensity of the Beads}

We know that the color of ferroin-loaded beads changes periodically when their oxidation state changes in the BZ reaction. In order to analyze the color change of a color image, the red, green and blue intensity (RGB) values are introduced to identify the color. As RGB values are not convenient for analysis, they have been converted to greyscale by the following empirical equation $[15,16]$ :

$$
\text { Greyscale }=0.299 \times \text { red }+0.587 \times \text { green }+0.114 \times \text { blue }
$$

Red, green and blue (RGB) represent the corresponding color components for each pixel of the image, the value ranging between 0 and 255. The calculated greyscale value represents the light intensity of that pixel. Therefore, the fraction of the light intensity for oxidized beads (blue beads) of an image can be calculated by the following equation:

$$
I_{i}=\frac{\left(S(G)_{i}-S(G)_{r e d}\right) / n_{i}}{\left(S(G)_{o x}-S(G)_{r e d}\right) / N}
$$

where $\bar{I}_{i}$ is the fraction light intensity of the oxidized beads for the $i$ th image, $S(G)_{i}$ is the sum of the greyscale values for all of the beads in the $i$ th image, and $S(G)_{\text {red }}$ and $S(G)_{o x}$ 


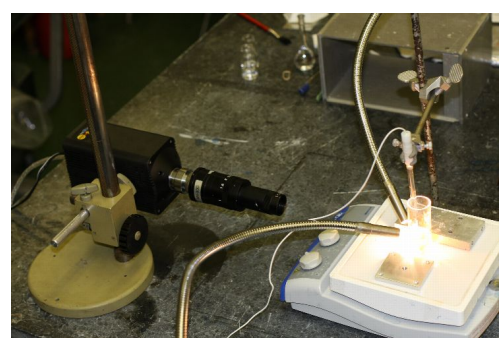

(a)

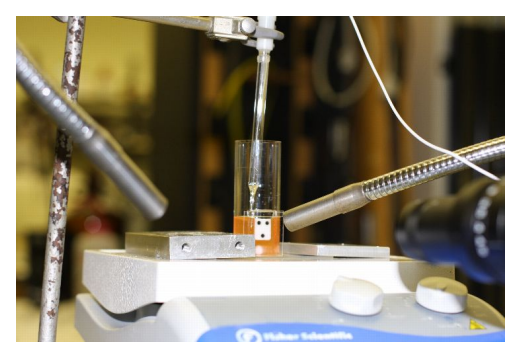

(b)

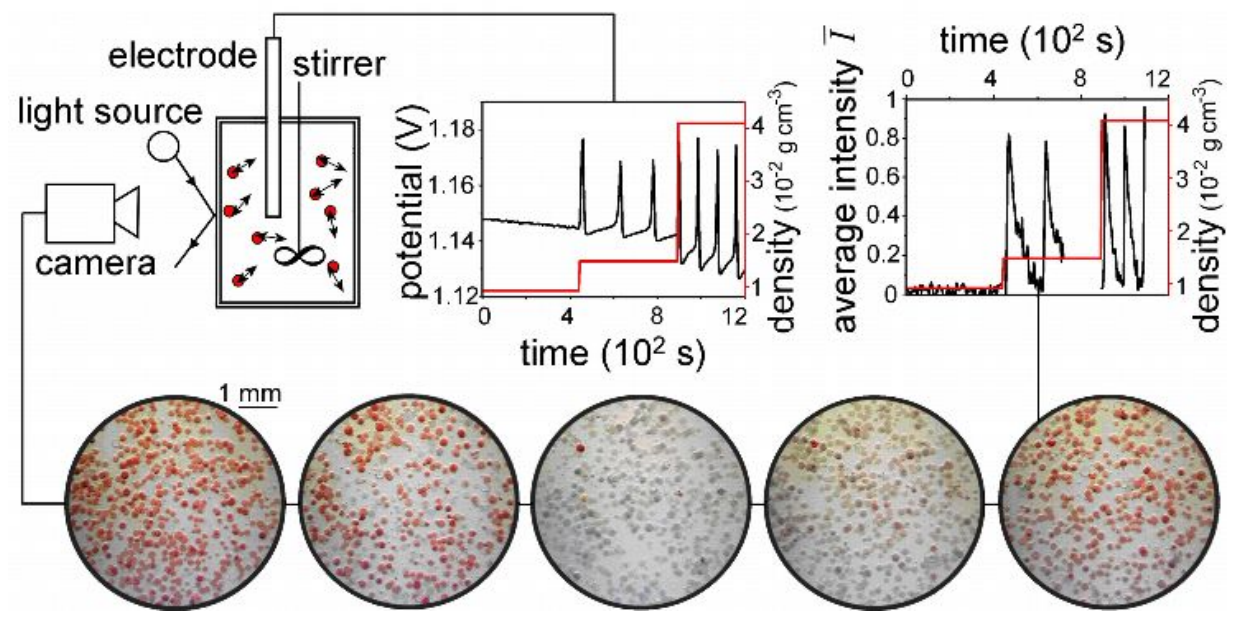

(c)

Figure 5.3. Experimental setup of a globally coupled system. (a) and (b) are two images of the instrumental setup, and (c) is a sketch of the experimental setup. Figure (c) shows that a microelectrode measures the oscillation amplitude as the potential changes. The normalized intensity in a series of images allows the average intensity of the beads as a function of time to be determined (right side). The red line in this figure represents the number density of the beads. The stirring speed is $600 \mathrm{rpm}$, and the images are recorded at a density of 0.02 $\mathrm{g} \mathrm{cm}^{-3}$, corresponding to approximately $1.3 \times 10^{4}$ beads $\mathrm{cm}^{-3}$. (Figure from Ref. [14].) 


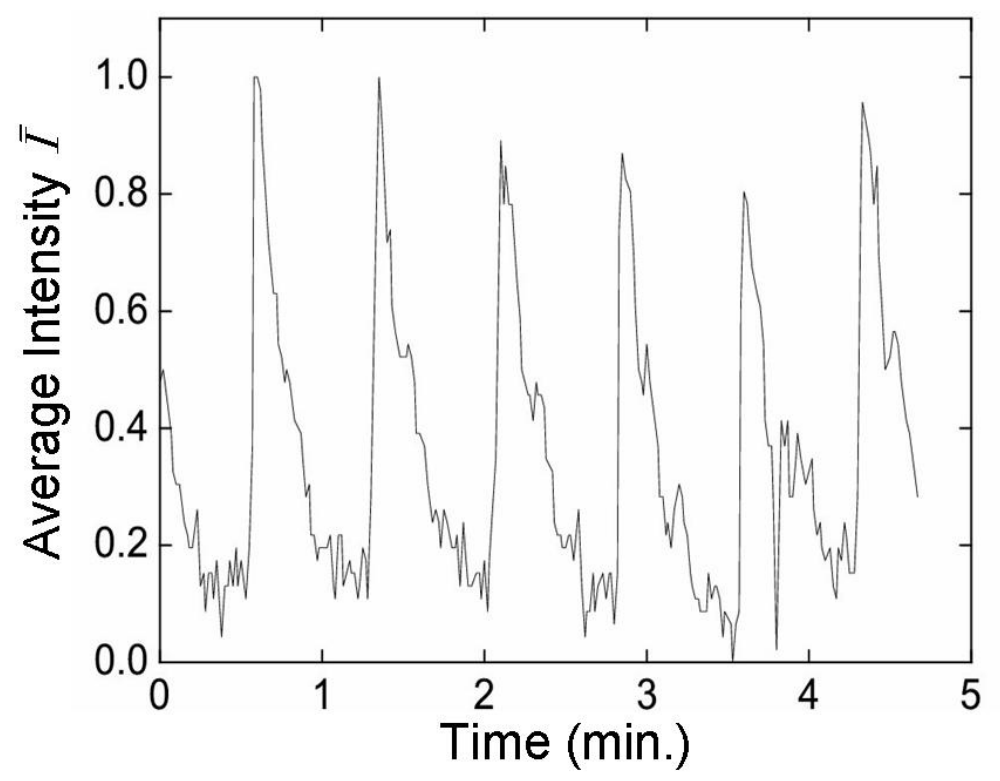

Figure 5.4. A typical plot of average intensity of the beads in the oscillatory states determined by image analysis. The number density of the beads is $0.05 \mathrm{~g} \mathrm{~cm}^{-1}$. The concentration of the solution: $[\mathrm{NaBr}]=0.07 \mathrm{M},[\mathrm{MA}]=0.14 \mathrm{M},\left[\mathrm{H}_{2} \mathrm{SO}_{4}\right]=0.67 \mathrm{M}$, and $\left[\mathrm{NaBrO}_{3}\right]=0.49$ M. The stirring rate is $600 \mathrm{rpm}$. (Figure reproduced from reference [14].)

are the sum of the greyscale values for all the beads when all the beads in the image are completely reduced (red) or oxidized (blue), respectively. $n_{i}$ is number of beads in the $i$ th image and $N$ is the number of beads in a reference image which all the beads are in completely oxidized or reduced state. $\bar{I}$ is average intensity and ranges from 0 (all beads red) to 1 (all beads blue). We obtained the time series of average light intensity of the beads and an example of oscillations of the average intensity is in Fig. 5.4. 


\subsection{Experimental Results}

Two distinct types of transitions to synchronization, which depend on the stirring speed and the number density of beads, have been observed in our experiment. One type of transition occurs at low stirring speed (300 rpm), another type occurs at high stirring speed (600 $\mathrm{rpm})$.

\subsubsection{Kuramoto Transition to Sychronization}

When the stirring speed is low (300 rpm), a Kuramoto transition is exhibited. As shown in Fig. 5.5(a), at low number density, the electrode potential signal of the solution is noisy and the period of oscillation cannot be identified. Although the average intensity of the beads does not show regular periodic behavior, there is a constant fraction of the beads in the excited state (0.2). This means about $20 \%$ of beads are in the oxidized state of the oscillatory cylcle. As the number density of beads is increased, oscillations of the global electrochemical potential begins with small amplitude. The amplitude gradually increases as the number density is increased. The average intensity of the beads shows the periodic oscillations and the maximum amplitude gradually increases. Figures 5.5(b) and 5.5(c) show the amplitude of the global electrochemical signal and the maximum average intensity as a function of the number density of beads, respectively. Figure 5.5(d) shows that the oscillation period decreases slightly as the number density of beads increases. 


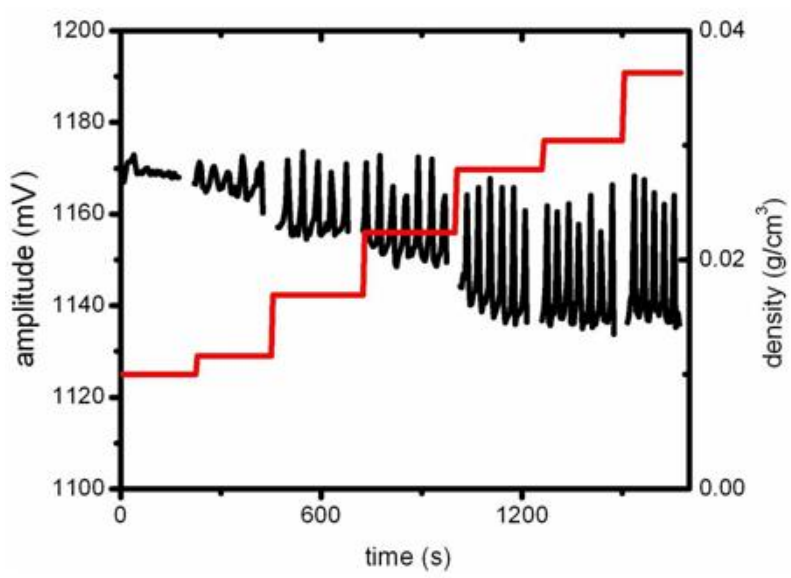

(a)

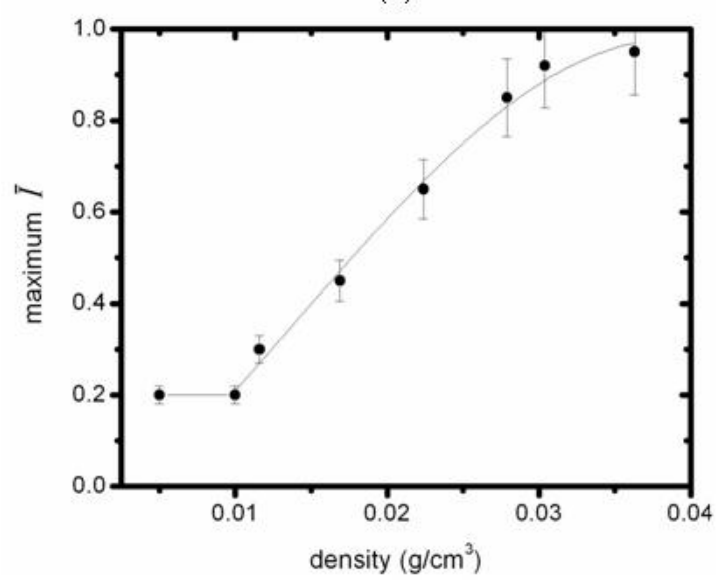

(c)

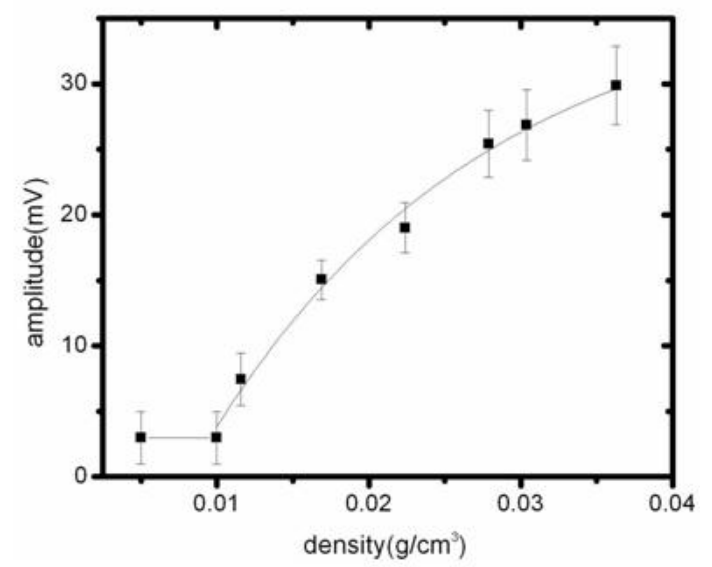

(b)

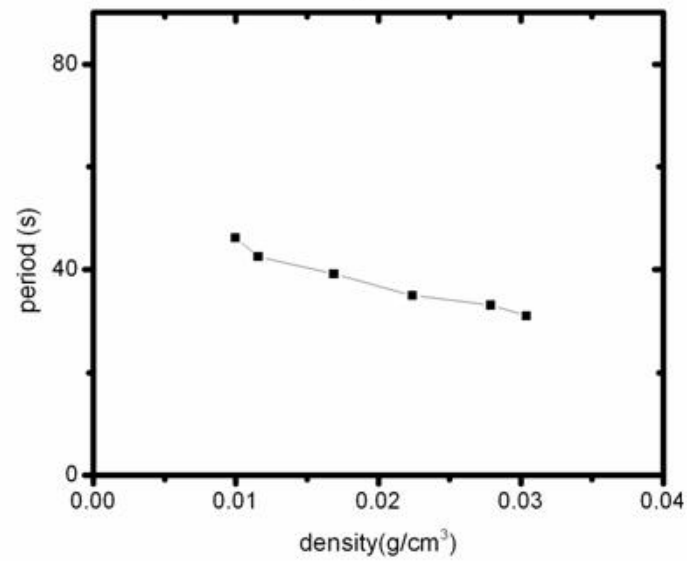

(d)

Figure 5.5. Kuramoto type transition to synchronization in the stirred system. Stirring speed is $300 \mathrm{rpm}$. The concentrations of the reactants: $\left[\mathrm{NaBrO}_{3}\right]=0.49 \mathrm{M},[\mathrm{MA}]=0.14 \mathrm{M}$, $\left[\mathrm{H}_{2} \mathrm{SO}_{4}\right]=0.67 \mathrm{M}$, and $[\mathrm{NaBr}]=0.07$. (a) Typical time series of the global electrode potential at different number density of beads. Black solid lines represent the electrical potential and the red solid step line represents the number density of beads. (b) The oscillatory amplitude of the global electrochemical potential, (c) the maximum average intensity $(\bar{I})$ of the associated images, and (d) the oscillation period as a function of number density of the beads. (Figure reproduced from reference [14, 17].) 


\subsubsection{Quorum Sensing Transition to Synchronization}

The other type of transition to synchronization occurs when the stirring speed is at 600 rpm, when a sharp change from steady state to oscillatory behavior can be observed as shown in Fig. 5.6. Figure 5.6(a) is a typical time series of the global electrochemical potential measured as a function of number density of beads. At low number density, the signal of the electrode potential remains at zero; no oscillatory signal can be measured in this state. The oxidized catalyst-loaded beads (blue beads) cannot be detected in the associated images. This steady state is maintained until the increasing number density reaches a threshold value, when a large amplitude oscillation of the electrode potential suddenly appears. The average intensity of the associated image also shows that nearly $80 \%$ of beads are oscillating at the same frequency, and they are oxidized simultaneously at each oscillation. Further increasing the number density produces a continuous increase in the amplitude of the global electrical potential, while the maximum value of the averge image intensity gradually approaches a constant value, as shown in Figs. 5.6(b) and 5.6(c). The oscillation period at high stirring speed is shown in Fig. 5.6(d). We see that the period of the oscillation decreases as the density of beads increases. We note that the period is larger than the period at low stirring speed.

Figures 5.5 and 5.6 characteristically describe the two distinct transitions to synchronization. For the Kuramoto transition, the state of the beads is always oscillatory; for the quorum sensing transition, the state of the beads is a stable steady state at low number den-

sity and oscillatory at high number density. As the number density increases, the amplitude of the oscillation gradually increases in the Kuramoto transition, while it suddently increases 
at the critical number density in the quorum sensing transition. The oscillation period for the Kuramoto transition decreases from $42 \mathrm{~s}$ to $32 \mathrm{~s}$ as the number density increases from 0.01 to $0.04 \mathrm{~g} \mathrm{~cm}^{-3}$, while for the quorum sensing transition, the oscillation period decreases from $150 \mathrm{~s}$ to $85 \mathrm{~s}$ in this density range. Figure 5.7 shows a series snapshots for these two transitions at low density (Fig. 5.7(a) and Fig. 5.7(c)) and snapshots of the Kuramoto transition at high density (Fig. 5.7(b)). Snapshots of the quorum sensing transition at high density are not shown here because the images are essentially the same as those shown in Fig. 5.7(b).

\subsection{Numerical Simulation}

\subsubsection{ZBKE Model}

From the Chapter 1, we described how the mechanism of the BZ reaction was studied by Field, Körös and Noyes (FKN) [18], and the Oregonator model [19], which gives the essential features of the FKN mechanism, was proposed. The ZBKE model was developed by Zhabotinsky, Buchholtz, Kiyatkin and Epstein in 1993 [20]. The ZBKE model improves the Oregonator model, and can more accurately describe the oscillatory behavior in the BZ reaction with the variation of parameters. The reaction scheme of the ZBKE model for the BZ system is as follows [20]:

$$
\begin{gathered}
\mathrm{H}^{+}+\mathrm{Br}^{-}+\mathrm{HOBr} \rightleftharpoons \mathrm{Br}_{2}+\mathrm{H}_{2} \mathrm{O} \\
\mathrm{H}^{+}+\mathrm{Br}^{-}+\mathrm{HBrO}_{2} \rightleftharpoons 2 \mathrm{HOBr}
\end{gathered}
$$




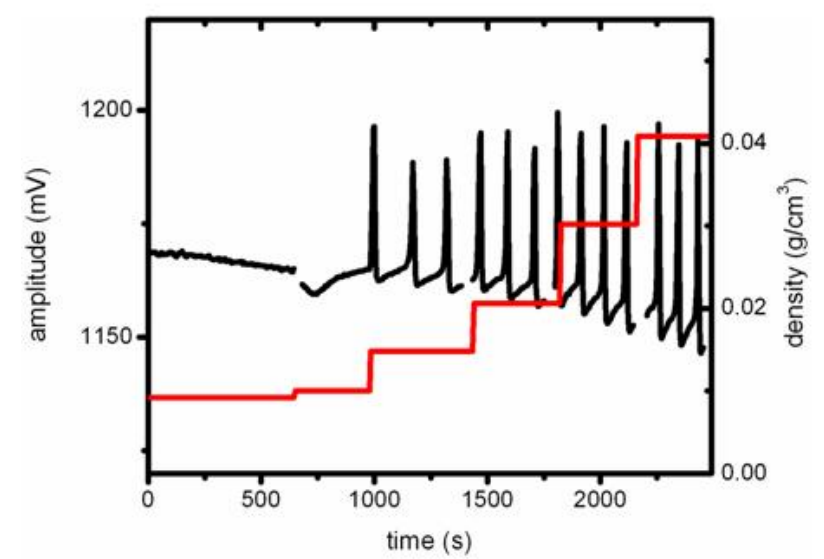

(a)

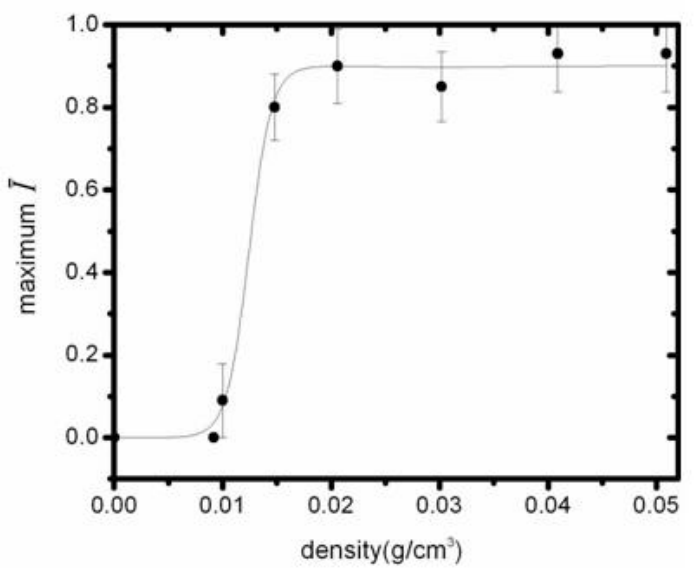

(c)

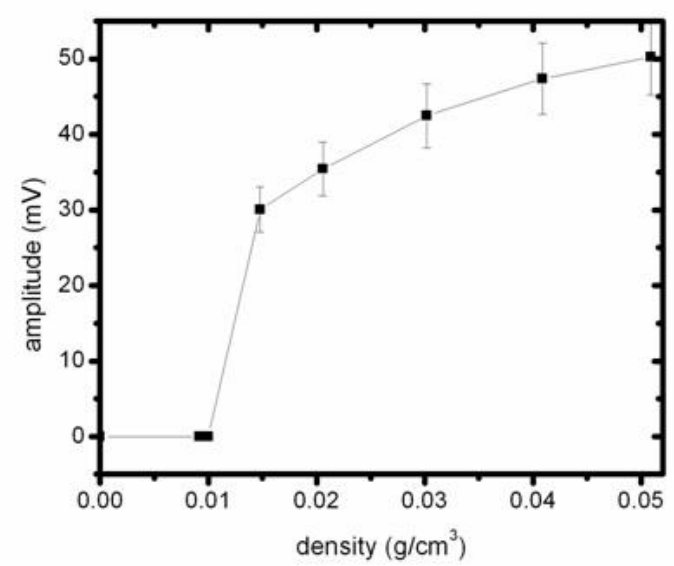

(b)

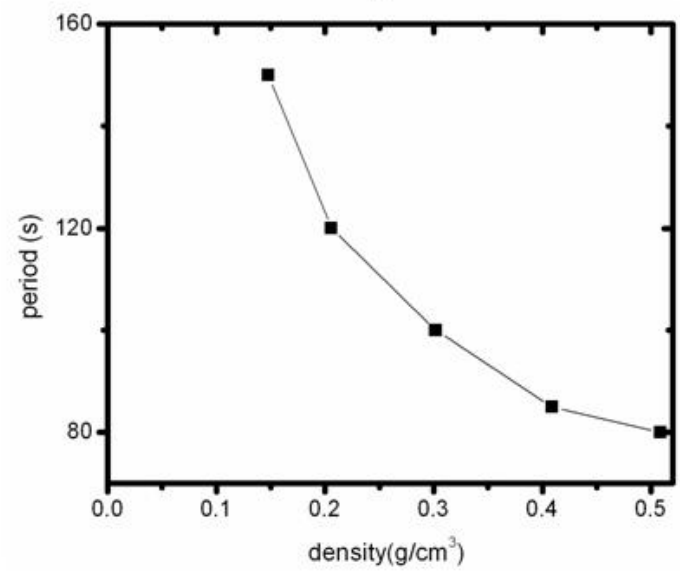

(d)

Figure 5.6. Quorum sensing type transition to synchronization in a stirred system. Stirring speed is $600 \mathrm{rpm}$. The concentrations of the reactants: $\left[\mathrm{NaBrO}_{3}\right]=0.49 \mathrm{M},[\mathrm{MA}]=0.14$ $\mathrm{M},\left[\mathrm{H}_{2} \mathrm{SO}_{4}\right]=0.67 \mathrm{M}$, and $[\mathrm{NaBr}]=0.07$. (a) A typical time series of the global electrode potential as a function of number density of beads. Black solid lines represent the electrical potential and red solid step line represents the number density of beads. (b) The oscillatory amplitude of the global electrochemical potential, (c) the maximum average intensity $(\bar{I})$ of the associated images, and (d) the oscillation period as a function of number density. (Figure reproduced from reference $[14,17]$.) 

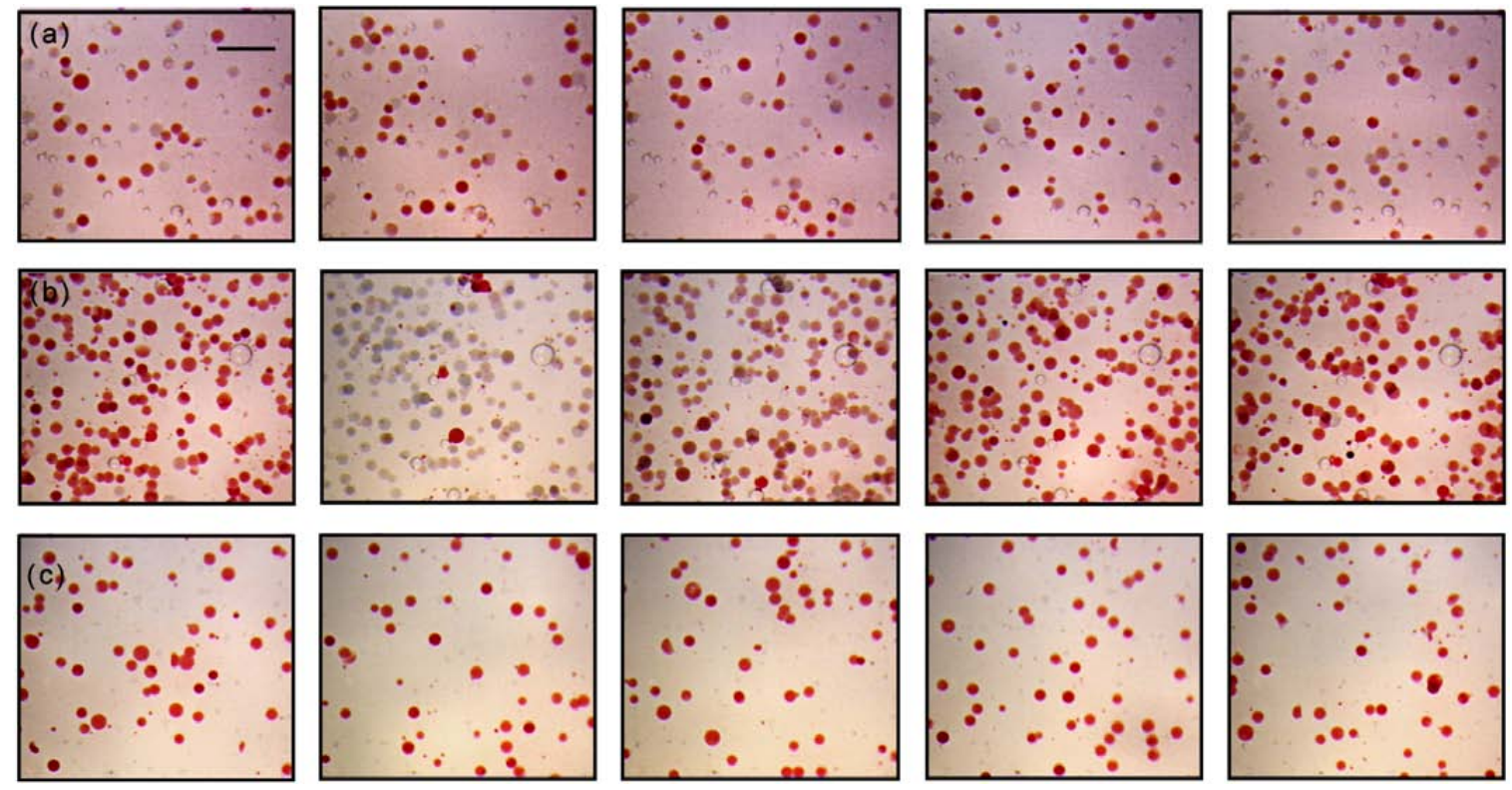

Figure 5.7. Snapshots of bead behavior at different stirring speeds and number density.

The images are taken at equal time intervals. (a) Stirring speed is $300 \mathrm{rpm}$ and the number density of beads is $0.01 \mathrm{~g} \mathrm{~cm}^{-3}$. We find a certain number of red beads (reduced state) and gray-blue beads (oxidized state) coexist in each snapshot, which indicates that the beads undergo desynchronized oscillations. Time interval for each image is $60 \mathrm{~s}$. (b) Stirring speed is $300 \mathrm{rpm}$ and the number density of beads is increased to $0.035 \mathrm{~g} \mathrm{~cm}^{-3}$. Almost all the beads show the same color in each snapshot indicating synchronized oscillations are exhibited. (c) Stirring speed is $600 \mathrm{rpm}$ and the number density of beads is $0.01 \mathrm{~g} \mathrm{~cm}^{-3}$. All the beads are red (reduced state) in each image indicating that they are in the steady state. Time interval for each image is $180 \mathrm{~s}$. The scale bar is $1.0 \mathrm{~mm}$. (Figure from reference [17].) 


$$
\begin{aligned}
& \mathrm{H}^{+}+\mathrm{Br}^{-}+\mathrm{HBrO}_{3} \rightleftharpoons \mathrm{HBrO}_{2}+\mathrm{HOBr} \\
& 2 \mathrm{HBrO}_{2} \rightleftharpoons \mathrm{HOBr}+\mathrm{HBrO}_{3} \\
& \mathrm{H}^{+}+\mathrm{HBrO}_{2} \rightleftharpoons 2 \mathrm{H}_{2} \mathrm{BrO}_{2}^{+} \\
& \mathrm{HBrO}_{2}+\mathrm{H}_{2} \mathrm{BrO}_{2}^{+} \rightleftharpoons \mathrm{HOBr}+\mathrm{HBrO}_{3}+\mathrm{H}^{+} \\
& \mathrm{H}^{+}+\mathrm{BrO}_{3}^{-} \rightleftharpoons \mathrm{HBrO}_{3} \\
& \mathrm{H}^{+}+\mathrm{HBrO}_{3}+\mathrm{HBrO}_{2} \rightleftharpoons \mathrm{HBrO}_{2}^{+}+\mathrm{BrO}_{2} \cdot+\mathrm{H}_{2} \mathrm{O} \\
& \mathrm{BrO}_{2} \cdot+\mathrm{H}^{+} \rightleftharpoons \mathrm{HBrO}_{2}^{+} \\
& \mathrm{M}_{\mathrm{red}}+\mathrm{HBrO}_{2}^{+} \rightleftharpoons \mathrm{M}_{\mathrm{ox}}+\mathrm{HBrO}_{2} \\
& \mathrm{M}_{\mathrm{ox}}+\mathrm{CHBr}(\mathrm{COOH})_{2} \rightleftharpoons \mathrm{M}_{\text {red }}+\mathrm{CBr}(\mathrm{COOH})_{2} \cdot+\mathrm{H}^{+} \\
& \mathrm{H}_{2} \mathrm{O}+\mathrm{CBr}(\mathrm{COOH})_{2} \cdot \rightarrow \mathrm{H}^{+}+\mathrm{Br}^{-}+\mathrm{COH}(\mathrm{COOH})_{2} . \\
& \mathrm{H}_{2} \mathrm{O}+\mathrm{CHBr}(\mathrm{COOH})_{2} \rightarrow \mathrm{CHOH}(\mathrm{COOH})_{2}+\mathrm{H}^{+}+\mathrm{Br}^{-} \\
& 2 \mathrm{COH}(\mathrm{COOH})_{2} \cdot \rightarrow \mathrm{CHOH}(\mathrm{COOH})_{2}+\mathrm{CO}(\mathrm{COOH})_{2} \\
& \mathrm{COH}(\mathrm{COOH})_{2} \cdot+\mathrm{CBr}(\mathrm{COOH})_{2} \cdot \rightarrow \mathrm{CHBr}(\mathrm{COOH})_{2}+\mathrm{CO}(\mathrm{COOH})_{2} \\
& \mathrm{HOBr}+\mathrm{CHBr}(\mathrm{COOH})_{2} \rightarrow \mathrm{CBr}_{2}(\mathrm{COOH})_{2}+\mathrm{H}_{2} \mathrm{O} \\
& \mathrm{Br}_{2}+\mathrm{CHBr}(\mathrm{COOH})_{2} \rightarrow \mathrm{CBr}_{2}(\mathrm{COOH})_{2}+\mathrm{H}^{+}+\mathrm{Br}^{-}
\end{aligned}
$$

In this mechanism, $\mathrm{M}_{\mathrm{ox}}$ is the oxidized form and $\mathrm{M}_{\mathrm{red}}$ is the reduced form of the catalyst. Here, $\mathrm{CBr}_{2}(\mathrm{COOH})_{2}, \mathrm{CHOH}(\mathrm{COOH})_{2}$ and $\mathrm{CO}(\mathrm{COOH})_{2}$ are considered to be final products. The reaction steps (RA-1) and (RA-2) are assumed to be fast so that other reactions that involve $\mathrm{HOBr}$ and $\mathrm{Br}_{2}$ as reactants are negligible. The equilibrium of reaction (R5c) is 
assumed to completely shift to the right in acidic conditions.

The mathematical model derived from the mechanism is:

$$
\begin{aligned}
\frac{d X}{d t} & =-k_{2} h_{0} X Y+k_{3} h_{0} X Y-2 k_{4}^{*} X^{2}-k_{5} h_{0} A X+k_{-5} U^{2}+k_{6} U(C-Z)-k_{-6} X Z \\
\frac{d Y}{d t} & =-k_{2} h_{0} X Y-k_{3} h_{0} A Y+k_{8}^{\prime} R_{1}+k_{9} B \\
\frac{d U}{d t} & =2 k_{5} h_{0} A X-2 k_{-5} U^{2}-k_{6} U(C-Z)+k_{-6} X Z \\
\frac{d Z}{d t} & =k_{6} U(C-Z)-k_{-6} X Z-k_{7} B Z+k_{-7} h_{0} R_{1}(C-Z), \\
\frac{d R_{1}}{d t} & =k_{7} B Z-k_{-7} h_{0} R_{1}(C-Z)-k_{8}^{\prime} R_{1}-k_{11} R_{1} R_{2} \\
\frac{d R_{2}}{d t} & =k_{8}^{\prime} R_{1}-2 k_{10} R_{2}^{2}-k_{11} R_{1} R_{2} .
\end{aligned}
$$

Here, $X=\left[\mathrm{HBrO}_{2}\right], Y=\left[\mathrm{Br}^{-}\right], U=\left[\mathrm{HBrO}_{2}^{+}\right], Z=\left[\mathrm{M}_{\mathrm{ox}}\right], R_{1}=\left[\mathrm{CBr}(\mathrm{COOH})_{2} \cdot\right], R_{2}=$ $\left[\mathrm{COH}(\mathrm{COOH})_{2} \cdot\right], A=\left[\mathrm{HBrO}_{3}\right]=\mathrm{h}_{0}\left[\mathrm{NaBrO}_{3}\right]_{0} /\left(0.2+\mathrm{h}_{0}\right), B=\left[\mathrm{CHBr}(\mathrm{COOH})_{2}\right], C=Z+$ $\left[\mathrm{M}_{\text {red }}\right], \mathrm{h}_{0}=$ Hammet acidity function, $k_{4}^{*}=k_{4}\left(1+0.87 h_{0}\right)$, and $k_{-5}=\left(k_{-5 b} k_{-5 c}\right) /\left(k_{5 c} h_{0}\right)$.

Assuming the radical reactions R10 and R11 are very fast, we can use the quasi-steadystate approximation for $R_{2}$. The equation of $R_{1}$ is then obtained as follows:

$$
d R_{1} / d t=k_{7} B Z-k_{-7} h_{0} R_{1}(C-Z)-\frac{k_{8}^{\prime} R_{1}}{q\left(R_{1}\right)}
$$

where

$$
\frac{1}{q\left(R_{1}\right)}=1-\frac{k_{11}^{2} R_{1}}{4 k_{8}^{\prime} k_{10}}\left(1-\left(1+\frac{8 k_{8}^{\prime} k_{10}}{k_{11}^{2} R_{1}}\right)^{1 / 2}\right)
$$

For radical reactions $\mathrm{R} 10$ and $\mathrm{R} 11$, if $\mathrm{R} 10$ is much faster than $\mathrm{R} 11$, then $q\left(R_{1}\right)=1.0$; if R11 is much faster than R10, then $q\left(R_{1}\right)=0.5$. We use $q$ as a substitute for $q\left(R_{1}\right)$, which can be varied from 0.5 to 1.0. We then define $k_{8}$ as $k_{8}=\frac{k_{8}^{\prime}}{q}$.

Assuming $R_{1}$ is a fast variable, when the quasi-steady-state approximation is applied, 
the equation system of the model can be reduced as follows:

$$
\begin{aligned}
\frac{d X}{d t} & =-k_{2} h_{0} X Y+k_{3} h_{0} X Y-2 k_{4}^{*} X^{2}-k_{5} h_{0} A X+k_{-5} U^{2}+k_{6} U(C-Z)-k_{-6} X Z \\
\frac{d Y}{d t} & =-k_{2} h_{0} X_{i} Y_{i}-k_{3} h_{0} X Y-k_{3} h_{0} A Y+q \frac{k_{7} k_{8} B Z}{k_{-7} h_{0}(C-Z)+k_{8}}+k_{9} B \\
\frac{d Z}{d t} & =k_{6} U(C-Z)-k_{-6} X Z-\frac{k_{7} k_{8} B Z}{k_{8}+k_{-7} h_{0}(C-Z)} \\
\frac{d U}{d t} & =2 k_{5} h_{0} A X-2 k_{-5} U^{2}-k_{6} U(C-Z)+k_{-6} X Z
\end{aligned}
$$

\subsubsection{The Relationship between Stirring Speed and}

\section{Chemical Exchange Rate}

The exchange of chemical species between the catalyst-loaded beads and the BZ solution is considered to be a reversible surface process [21]. The exchange rate $(v)$ is described as a function of the stirring speed and is directly related to the concentration difference of species at the bead and in the solution:

$$
v=-k_{e x}\left(X_{i}-X_{s}\right)
$$

where $X_{i}$ and $X_{s}$ are the concentrations of species at the bead and in the solution, respectively. The exchange rate constant $k_{e x}$ depends on the surface area $(A)$ and volume $(V)$ of the bead:

$$
k_{e x}=-k_{s l} \frac{A}{V}
$$

Here, $k_{s l}$ is the solid to liquid mass transfer coefficient [22]. When stirring speed is at zero, $k_{s l}$ is only related to the diffusion coefficient. When stirring takes place, $k_{s l}$ is a complex function which depends on the stirring method and the stirring speed. The values of $k_{s l}$ are generally 
in the range of $10^{-3}$ and $10^{-1} \mathrm{~cm} \mathrm{~s}^{-1}$, and $k_{s l}$ increases monotonically with increasing stirring rate [22]. Hence, we can vary the chemical exchange rate by changing the stirring speed.

\subsubsection{A Model of the Globally Coupled Oscillatory Bead System}

In order to analyze these transitions to synchronized oscillatory behavior, a modified three-variable ZBKE model is implemented for the ferroin-catalyzed BZ reaction. In our BZ reaction system, there exists a species exchange between the catalyst-loaded beads and the surrounding solution. A sketch of the species exchange is shown in Fig. 5.8. $X$ represents the concentration of the activator $\mathrm{HBrO}_{2}, Y$ the inhibitor $\mathrm{Br}^{-}$, and $Z$ the oxidized form of the catalyst $\mathrm{Fe}(\mathrm{phen})_{3}^{3+}$. Each bead in the $\mathrm{BZ}$ solution is treated as a mini $\mathrm{BZ}$ reaction system, and each has its own species concentrations $\left(X_{i}, Y_{i}\right.$ and $\left.Z_{i}\right)$. The surrounding solution is catalyst-free and there only two concentrations $\left(X_{s}\right.$ and $\left.Y_{s}\right)$ need to be considered. The concentrations of $\mathrm{HBrO}_{2}$ for the $i$ th bead and the surrounding solution are:

$$
\begin{gathered}
\frac{d X_{i}}{d t}=-k_{e x}\left(X_{i}-X_{s}\right)+f\left(X_{i}, Y_{i}, Z_{i}\right), \\
\frac{d X_{s}}{d t}=\frac{\bar{V}}{V_{s}} \sum_{i=1}^{N} k_{e x}\left(X_{i}-X_{s}\right)+g\left(X_{s}, Y_{s}\right) .
\end{gathered}
$$

Here, $f\left(X_{i}, Y_{i}, Z_{i}\right)$ represents the chemical reaction on the beads, and $g\left(X_{s}, Y_{s}\right)$ represents the chemical reaction in the surrounding solution, which is given by the ZBKE model [20]. $k_{e x}\left(X_{i}-X_{s}\right)$ is the exchange rate of $\mathrm{HBrO}_{2}$ between the $i$ th bead and the surrounding solution as previously described. $k_{e x}$ is the exchange rate constant, which increases with

increasing stirring rate [22]. $\frac{\bar{V}}{V_{s}}$ is the dilution factor, where $V_{s}$ and $\bar{V}$ are the total volume of the solution and the average volume of a single bead, respectively. 


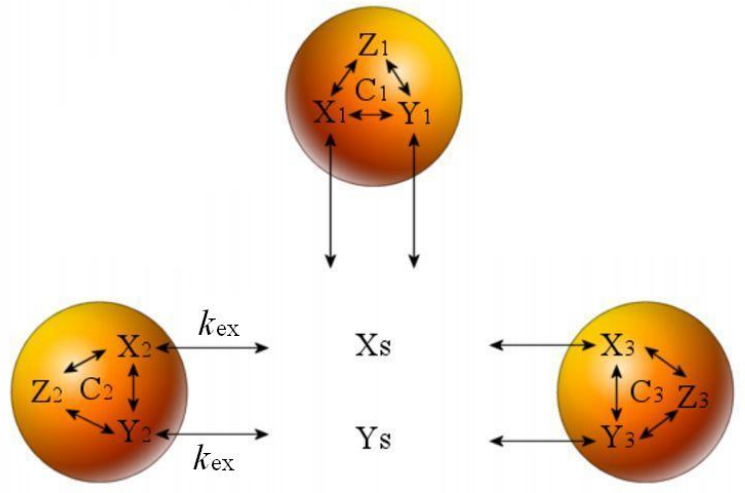

Figure 5.8. A sketch of the species exchange between the beads and the surrounding solution. Each bead is considered to be a mini BZ reaction system, which exchanges $\mathrm{HBrO}_{2}$ (activator $X$ ) and $\mathrm{Br}^{-}$(inhibitor $Y$ ) with the surrounding solution. The catalyst $(C$ ) only exists on the beads, not in the solution. $Z$ is the oxidized form of catalyst, and $k_{e x}$ is the exchange rate constant related to the stirring speed. (Figure from Ref. [23].)

From the reduced ZBKE model, we obtain the four-variable differential equations of the oscillatory bead system. In our ferroin-catalyzed BZ system, the intermediate $\mathrm{HBrO}_{2}^{+}(U)$ has a steady-state concentration $\left(U_{s s}\right)$, which is given by:

$$
U_{s s}=\frac{1}{4 k_{-5}}\left(-k_{6}\left(C-Z_{i}\right)+\left(k_{6}^{2}\left(C-Z_{i}^{2}\right)+16 k_{-5} k_{5} h_{0} A x_{i}+8 k_{-5} k_{-6} x_{i} z_{i}\right)^{1 / 2}\right) .
$$

The concentrations $X, Y$ and $Z$ on the $i$ th bead are given by:

$$
\begin{gathered}
\frac{d X_{i}}{d t}=-k_{e x}\left(X_{i}-X_{s}\right)-k_{2} h_{0} X_{i} Y_{i}+k_{3} h_{0} A Y_{i}-2 k_{4} X_{i}^{2}-k_{5} h_{0} A X_{i} \\
+k_{-5} U_{s s}^{2}+k_{6} U_{s s}\left(C-Z_{i}\right)-k_{-6} X_{i} Z_{i} \\
\frac{d Y_{i}}{d t}=-k_{e x}\left(Y_{i}-Y_{s}\right)-k_{2} h_{0} X_{i} Y_{i}-k_{3} h_{0} A Y_{i}+q_{i} \frac{k_{7} k_{8} B Z_{i}}{k_{-7} h_{0}\left(C-Z_{i}\right)+k_{8}}+k_{9} B
\end{gathered}
$$




$$
\frac{d Z_{i}}{d t}=k_{6} U_{s s}\left(C-Z_{i}\right)-k_{-6} X_{i} Z_{i}-\frac{k_{7} k_{8} B Z_{i}}{k_{-7} h_{0}\left(C-Z_{i}\right)+k_{8}}
$$

and in the surrounding solution, the concentrations $X_{s}$ and $Y_{s}$ are:

$$
\begin{gathered}
\frac{d X_{s}}{d t}=\frac{\bar{V}}{V_{s}} \sum_{i=1}^{N} k_{e x}\left(X_{i}-X_{s}\right)-k_{2} h_{0} X_{s} Y_{s}+k_{3} h_{0} A Y_{s}-2 k_{4} X_{s}^{2}+k_{-5} U_{s s}^{2} \\
\frac{d Y_{s}}{d t}=\frac{\bar{V}}{V_{s}} \sum_{i}^{N} k_{e x}\left(Y_{i}-Y_{s}\right)-k_{2} h_{0} X_{s} Y_{s}-k_{3} h_{0} A Y_{s}+k_{9} B
\end{gathered}
$$

Here, we see that the dynamics of the beads depends on the exchange rate $k_{e x}$ and the number density of the beads. The oscillation in the surrounding solution appears only in the presence of oscillatory beads, and the oscillations of $X_{s}$ and $Y_{s}$ are generated from the species exchange between the beads and solution.

The three-variable ZBKE model is applied in our simulations, where the equations are integrated using the Euler method with $d t=0.001 \mathrm{~s}$. All the parameters used in the simulations for the ferroin-catalyzed BZ system are listed in Table 6.1.

\subsubsection{Order Parameter}

In order to quantitatively describe the transition to synchronization of the oscillators, Shinomoto and Kuramoto define an order parameter $K$ as [24]:

$$
K=<\left|N^{-1} \sum e^{i \theta_{j}}-<N^{-1} \sum e^{i \theta_{j}}>\right|>
$$

where $N$ is the number of oscillators ( $N=1000$ in our simulation), $\theta_{j}$ is the phase of the $j$ th oscillator, and $\langle>$ represents the average over time. $K=0$ means that the oscillators have random phases, and $K=1$ means the oscillations are in perfect synchrony. If the oscillations 
Table 5.1. The parameter values in the ferroin-catalyzed ZBKE model [14]

\begin{tabular}{|c|c|c|c|c|c|}
\hline Parameter & Value & Parameter & Value & Parameter & Value \\
\hline $\mathrm{A}(\mathrm{M})$ & 0.39 & $k_{2}\left(\mathrm{M}^{-2} \mathrm{~s}^{-1}\right)$ & $7.6 \times 10^{6}$ & $k_{-6}\left(\mathrm{M}^{-1} \mathrm{~s}^{-1}\right)$ & 0.30 \\
$\mathrm{~B}(\mathrm{M})$ & 0.14 & $k_{3}\left(\mathrm{M}^{-2} \mathrm{~s}^{-1}\right)$ & 2.0 & $k_{8} / k_{-7}\left(\mathrm{M}^{2}\right)$ & $3.0 \times 10^{-6}$ \\
$\mathrm{C}(\mathrm{M})$ & $1.7 \times 10^{-3}$ & $k_{4}\left(\mathrm{M}^{-1} \mathrm{~s}^{-1}\right)$ & 8600 & $k_{8} k_{7} / k_{-7}\left(\mathrm{M} \mathrm{s}^{-1}\right)$ & $5.0 \times 10^{-7}$ \\
$h_{0}$ & 0.77 & $k_{5}\left(\mathrm{M}^{-2} \mathrm{~s}^{-1}\right)$ & 10.0 & $k_{9}\left(\mathrm{~s}^{-1}\right)$ & $3.3 \times 10^{-6}$ \\
$\bar{q}$ & 0.6 & $k_{-5}\left(\mathrm{M}^{-1} \mathrm{~s}^{-1}\right)$ & $4.2 \times 10^{6}$ & $k_{e x}\left(\mathrm{~s}^{-1}\right)$ & $0.30-30$ \\
$\sigma_{q}$ & 0.05 & $k_{6}\left(\mathrm{M}^{-1} \mathrm{~s}^{-1}\right)$ & $1.66 \times 10^{7}$ & $\bar{V} / V_{s}$ & $1.0 \times 10^{-6}$ \\
\hline
\end{tabular}

are partially synchronized, then $K$ is between 0 and 1.

\subsubsection{Simulation Results}

Based on the three-variable ZBKE model, we obtain a surface plot of the concentration amplitude $\left(X_{s}\right)$ as a function of the exchange rate $k_{e x}$ and the number density of beads $n$ (Fig. 5.9(a)), and a surface plot of the order parameter $K$ versus $k_{e x}$ and $n$ (Fig. 5.9(b)). In Fig. 5.9(a), it is easy to find the characteristic areas of the quorum sensing and the Kuramoto transitions on the plot. $k_{e x}$ monotonically increases with increasing stirring speed, and therefore high stirring speed means high $k_{e x}$. The quorum sensing transition occurs in this region. As the green line shows, the bromous acid concentration in the solution $\left(X_{s}\right)$ remains very low at high $k_{e x}$ and low number density of beads, and the beads in this area are in the steady state. With the number density $n$ increasing to a threshold value, a sudden 
jump in the global concentration $X_{s}$ occurs. The order parameter $K$ similarly changes (see the same area in Fig. 5.9(b)). Therefore, there exists either a steady state or a perfectly synchronized oscillatory state at different number densities $(n)$ at high $k_{e x}$ (Fig. 5.10(a)).

On the other hand, low $k_{e x}$ means low stirring speed, and the Kuramoto transition occurs in this region in Fig. 5.9(a). As the red arrows show, the bromous acid concentration gradually increases when the number density of beads is increased, and the corresponding order parameter $K$ also gradually increases (see the same area in Fig. 5.9(b)). In Fig. 5.9(b), we see that the order parameter $K$ is not equal to 0 like $X_{s}$ at low bead density, which means that most of the beads are still in the oscillatory state. However, the period distribution of the beads at this density is wide, which gradually decreases with the increasing number density $n$ (see Fig. 5.10(b)).

Our model also predicts that a desynchronization transition will occur as the exchange rate is reduced. This has also been observed in the experiment, as shown in Fig. 5.11(a). After a quorum-sensing transition, a synchronized oscillation with high amplitude suddenly appears, and almost all of the beads are in synchrony. The amplitude of the global concentration $X_{s}$ gradually decreases as $k_{e x}$ is reduced until only a noise-like signal is observed, which means the oscillations of the beads are at random phases. This transition occurs at low density of the beads as shown in Fig. 5.9(a).

In the model, the concentration $X_{s}$ plays an important role for the mean-field coupling. Figure 5.11(b) shows the concentration of bromous acid on a single bead $\left(X_{i}\right)$, in the solution $\left(X_{s}\right)$, and the exchange for one oscillatory cycle. From Fig. 5.11(b), we see that the average exchange rate over one cycle for all the beads is negative; hence, we take its absolute value as a loss rate. Figure 5.11(c) shows that, for a fixed number of beads $n$, the average concentra- 
tion of bromous acid in the surrounding solution $\left(<X_{s}>\right)$ increases as $k_{e x}$ is increased, and the corresponding loss rate also increases; therefore, the average concentration on a bead $\left(<\bar{X}_{i}>\right)$ decreases.

Figure 5.11(d) shows $\left\langle X_{s}\right\rangle,\left\langle\bar{X}_{i}\right\rangle$, and the loss rate at a different number densities $n$ when the exchange rate constant $k_{e x}$ is high. At high $k_{e x}$ and low $n$, all of the beads are quiescent. The loss rate is high because of the low $<X_{s}>$. As $n$ increases, both $<X_{s}>$ and $<\bar{X}_{i}>$ increase. When $n$ reaches a critical value, $X_{i}$ reaches the threshold for the transition from the quiescent state to oscillations. Both $\left\langle X_{s}>\right.$ and $\left\langle\bar{X}_{i}\right\rangle$ undergo a sudden jump, which leads to a large increase in the loss rate.

\subsection{Summary}

As the number density of beads increases, two distinct types of transitions, the quorum sensing transition and the Kuramoto transition to synchronization have been observed in a globally coupled oscillator system. The coupling occurs through the species exchange between the oscillators and their surrounding solution, which is described with an exchange rate. At low stirring speed, the exchange rate is low, and the oscillations of beads are gradually synchronized as the number density of beads increases. This is the Kuramoto transition to synchronization. If the stirring speed is high, the exchange rate is high and the coupling strength between the beads and the surrounding solution is strong. The beads are quiescent if the number density is lower than a critical value. At a critical value of number density, synchronized oscillations suddenly appear. This is the quorum sensing transition to synchro- 


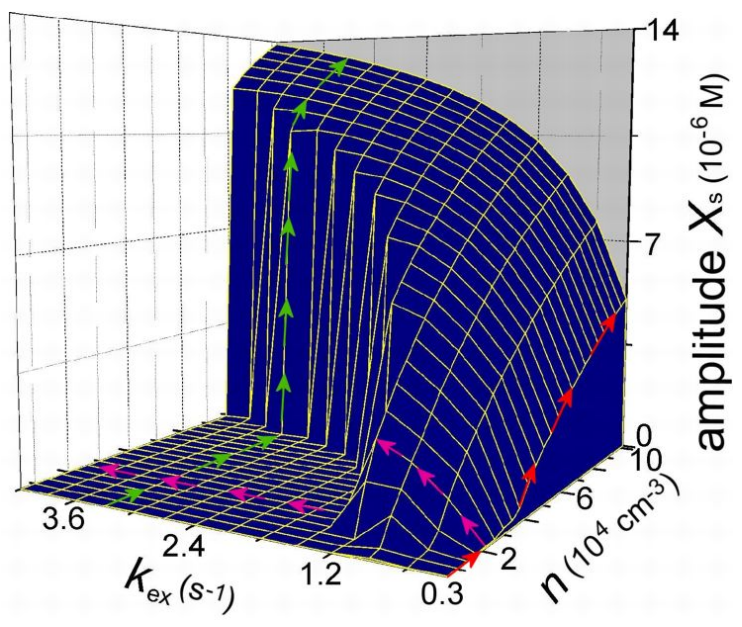

(a)

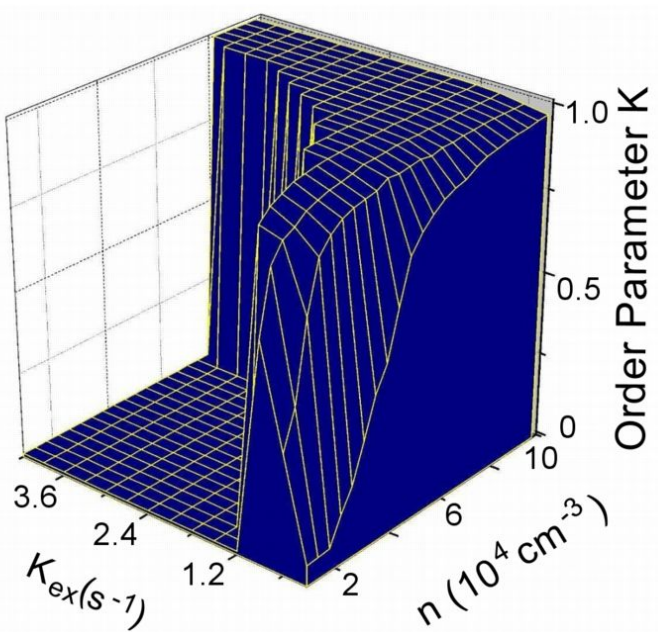

(b)

Figure 5.9. Surface plots of bromous acid concentration $X_{s}$ and the order parameter $K$. (a) A three-dimensional surface plot of bromous acid concentration $\left(X_{s}\right)$ versus density $n$ and exchange rate constant $k_{e x}$. Kuramoto synchronization occurs at low exchange rate and the quorum sensing transition at high exchange rate, as shown by the red and green arrows. The pink arrows indicate that at a constant number density, the bromous acid concentration $X_{s}$ gradually increases as the exchange rate constant $k_{e x}$ increases. When $k_{e x}$ reaches a threshold value, $X_{s}$ falls to nearly zero, corresponding to the steady state. (b) The order parameter $K$ at the same conditions as in plot (a). (Figure from Ref. [14].) 

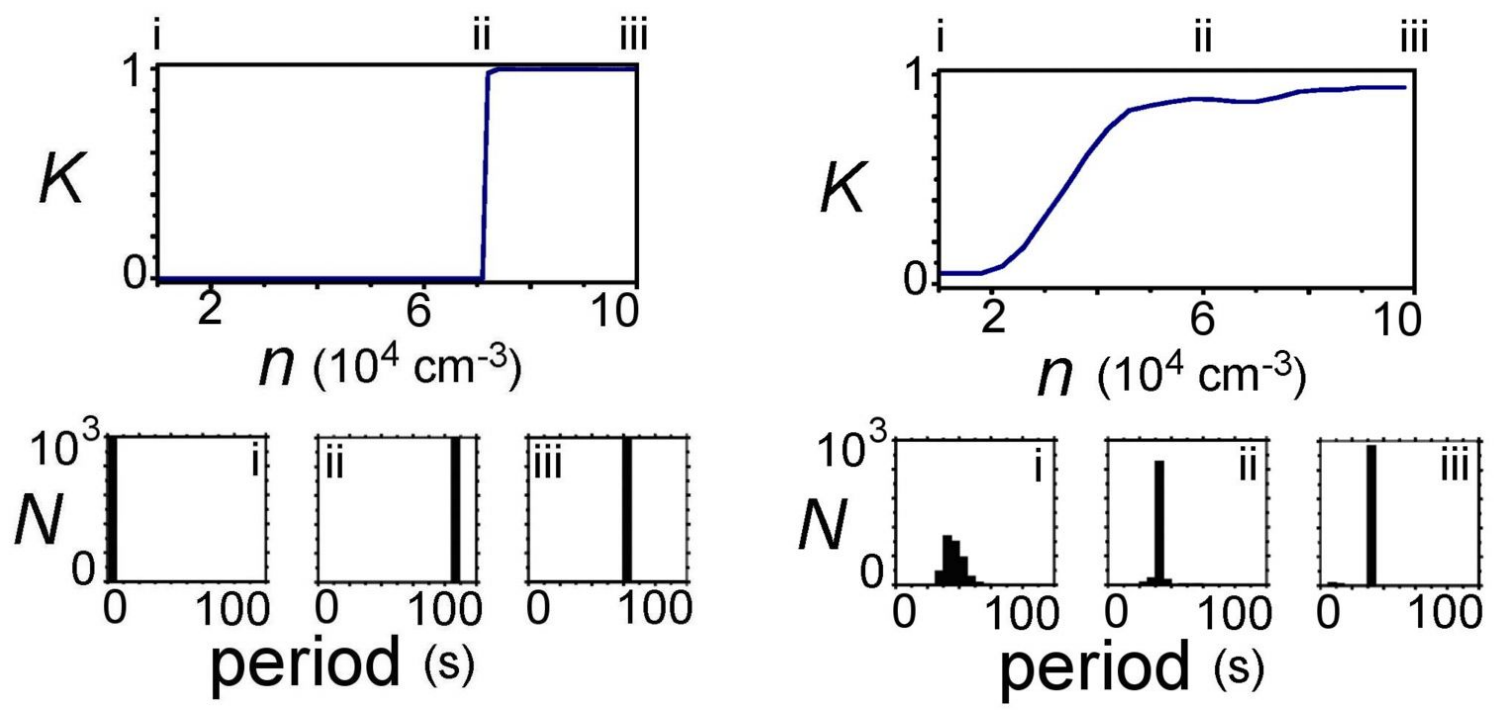

(a)

(b)

Figure 5.10. Order parameter $K$ versus the number density and the corresponding period distribution of the beads in the Kuramoto or quorum sensing transitions. (a) Order parameter $K$ as versus number density $n$ in the quorum sensing transition. Bottom panels show the period distributions at different number densities: (i) $n=6 \times 10^{4} \mathrm{~cm}^{-3}$, (ii) $8 \times 10^{4} \mathrm{~cm}^{-3}$, and (iii) $10 \times 10^{4} \mathrm{~cm}^{-3}$. (b) Order parameter $K$ versus number density $n$ in the Kuramoto transition. Bottom panels show the period distributions at different number densities (i) $n$ $=2 \times 10^{4} \mathrm{~cm}^{-3}$, (ii) $6 \times 10^{4} \mathrm{~cm}^{-3}$, and (iii) $10 \times 10^{4} \mathrm{~cm}^{-3}$. (Figure from Ref. [14].) 


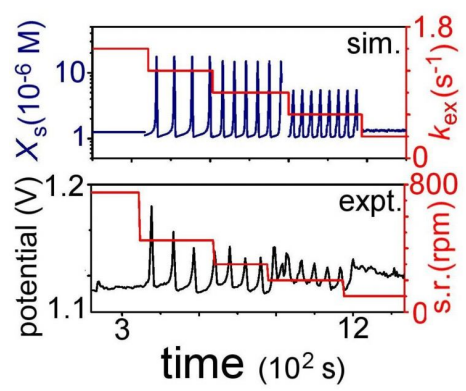

(a)

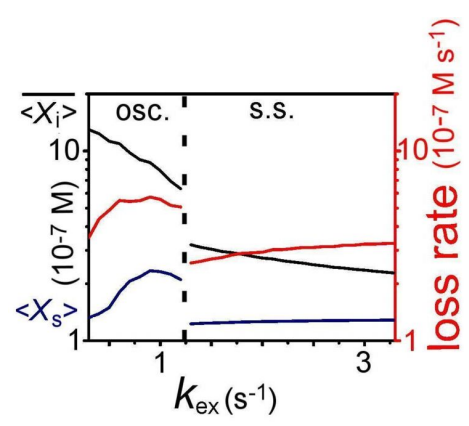

(c)

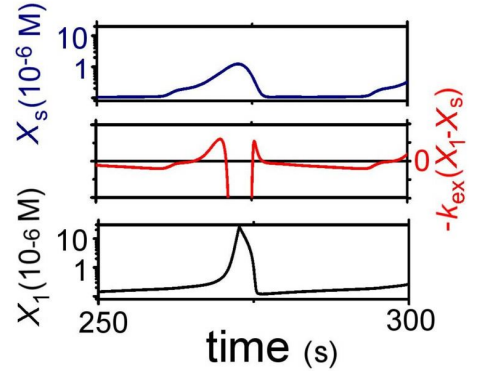

(b)

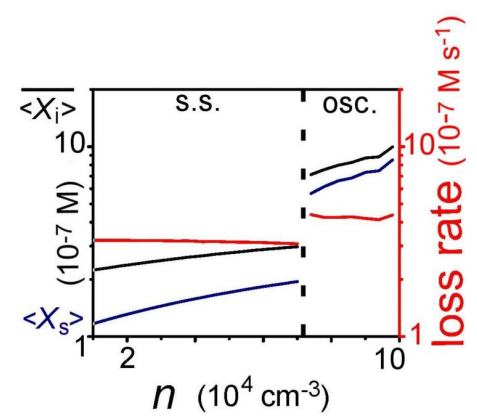

(d)

Figure 5.11. The influence of the exchange rate $-k_{e x}\left(X_{i}-X_{s}\right)$ on the transitions to synchronization. (a) Time-series showing sdesynchronized behavior of beads as $k_{e x}$ (in simulation, $n=1.8 \times 10^{4} \mathrm{~cm}^{-3}$ ) or stirring speed (in experiment, density $=0.0162 \mathrm{~g} \mathrm{~cm}^{-3}$ ) is decreased. (b) Time-series concentrations of bromous acid on a single bead $\left(X_{i}=\right.$ black line), in the solution $\left(X_{s}=\right.$ blue line), and the exchange rate $-k_{e x}\left(X_{i}-X_{s}\right)$ (red line) in one oscillatory cycle. The conditions for this calculation are $n=1.8 \times 10^{4} \mathrm{~cm}^{-3}$ and $k_{e x}=0.3$ $\mathrm{s}^{-1}$. (c) The time-averaged activator concentration on the beads $\left(<\bar{X}_{i}\right\rangle=$ black line), in the solution $\left(<X_{s}\right\rangle=$ blue line $)$, and the loss rate from beads $\left(<k_{e x}\left(\bar{X}_{i}-X_{s}\right)>=\right.$ red line) as $k_{e x}$ is increased. The transition is from the oscillatory state to the steady state with increasing $k_{e x}\left(n=1.8 \times 10^{4} \mathrm{~cm}^{-3}\right)$. (d) The transition is from the steady state to the oscillatory state with increasing $n\left(k_{e x}=3.0 \mathrm{~s}^{-1}\right)$. Color of lines same as in (c). (Figure from Ref. [14].) 
nization.

Numerical simulations based on the three-variable ZBKE model [20] have been carried out. Two 3-D surface plots describe two types of relationships, one between the activator concentration in the solution $X_{s}$ with $k_{e x}$ and density $n$, and the other between the order parameter $K$ with $k_{e x}$ and density $n$. We analyze the time series of the activator concentration on the bead, in the solution, and the corresponding exchange rate for one cycle. We also calculate the time-averaged activator concentrations on the bead, in the solution, and corresponding loss rate with $k_{e x}$ increasing as density $n$ is fixed or with density $n$ increasing with $k_{e x}$ fixed. The calculation shows the transition form the oscillatory state to a steady state, and from the steady state to an oscillatory state. 


\section{Reference}

[1] J. Buck, "Synchronous rhythmic flashing of fireflies. II." Q. Rev. Biol. 63, 265-289 (1988).

Online Version 71

[2] P. Richard, "The rhythm of yeast," FEMS Microbiol. Rev. 27, 547-557 (2003). Online Version 71

[3] L. Glass, "Synchronization and rhythmic processes in physiology," Nature 410, 277-284 (2001).

Online Version 71

[4] A. T. Winfree, "Biological rhythms and the behavior of populations of coupled oscillators," J. Theor. Biol. 16, 15-42 (1967).

Online Version 71

[5] Y. Kuramoto, "Self-entrainment of a population of coupled nonlinear oscillators," in H. Araki, ed., "In International Symposium on Mathematical Problems in Theoretical Physics," volume 39 of Lecture Notes in Physics, Kyoto University (Springer, 1975). 71

[6] G. Gerisch and B. Hess, "Cyclic-amp-controlled oscillations in suspended dictyostelium cells: their relation to morphogenetic cell interactions," Proc. Natl. Acad. Sci. U. S. A. 71, 2118-2122 (1974).

Online Version 72

[7] I. Z. Kiss, Y. Zhai, and J. L. Hudson, "Emerging coherence in a population of chemical 
oscillators," Science 296, 1676-1678 (2002).

Online Version 72

[8] J. Aldridge and E. K. Pye, "Cell density dependence of oscillatory metabolism," Nature 259, $670-671(1976)$.

Online Version 72

[9] A. Camilli and B. L. Bassler, "Bacterial small-molecule signaling pathways," Science 311, $1113-1116$ (2006).

Online Version 72

[10] E. P. Greenberg, "Quorum sensing in gram-negative bacteria," ASM News 63, 371377 (1997). 72

[11] A. Eberhard, A. L. Burlingame, C. Eberhard, G. L. Kenyon, K. H. Nealson, and N. J. Oppenheimer, "Structural identification of autoinducer of photobacterium fischeri luciferase," Biochemistry 20, 2444-2449 (1981).

Online Version

[12] J. Engebrecht, K. Nealson, and M. Silverman, "Bacterial bioluminescence: Isolation and genetic analysis of functions from Vibrio fischeri," Cell 32, 773-781 (1983). Online Version 72

[13] G. M. Patriquin, E. Banin, C. Gilmour, R. Tuchman, E. P. Greenberg, and K. Poole, "Influence of quorum sensing and iron on twitching motility and biofilm formation in pseudomonas aeruginosa," J. Bacteriol. 190, 662-671 (2008). Online Version 72 
[14] A. F. Taylor, M. Tinsley, F. Wang, Z. Huang, and K. Showalter, "Dynamical quorum sensing and synchronization in large populations of chemical oscillators," Science $\mathbf{3 2 3}$, $614-617$ (2009).

Online Version ix, 77, 79, 80, 82, 85, 93, 96, 97, 98

[15] C. Poynton, Digital video and HDTV algorithms and interfaces (Morgan Kaufmann, San Francisco, USA, 2003).

Online Version 78

[16] W. Wharton and D. Howorth, Principles of Television Reception (Pitman Publishing, 1971). 78

[17] M. Tinsley, A. F. Taylor, Z. Huang, F. Wang, and K. Showalter, "Dynamical quorum sensing and synchronization in collections of excitable and oscillatory catalytic particles," Physica D 239, 785-790 (2010).

Online Version 82, 85, 86

[18] R. J. Field, E. Körös, and R. M. Noyes, "Oscillations in chemical systems. I. detailed mechanism in a system showing temporal oscillations," J. Am. Chem. Soc. 94, 1394$1396(1972)$.

Online Version 84

[19] R. J. Field and R. M. Noyes, "Oscillations in chemical systems. IV. limit cycle behavior in a model of a real chemical reaction," J. Chem. Phys. 60, 1877-1884 (1974). Online Version 84

[20] A. M. Zhabotinsky, F. Buchholtz, A. B. Kiyatkin, and I. R. Epstein, "Oscillations and 102 
waves in metal-ion-catalyzed bromate oscillating reactions in highly oxidized states," J. Phys. Chem. 97, 1518-7584 (1993).

Online Version 84, 90, 99

[21] L. D. Schmidt, The Engineering of Chemical Reactions (Oxford Univeristy Press, 1998). 89

[22] V. G. Pangarkar, A. A. Yawalkar, M. M. Sharma, and A. A. C. M. Beenackers, "Particleliquid mass transfer coefficient in two-/three-phase stirred tank reactors," Ind. Eng. Chem. Res. 41, 4141-4167 (2002).

Online Version 89, 90

[23] R. Toth, A. F. Taylor, and M. R. Tinsley, "Collective behavior of a population of chemically coupled oscillators," J. Phys. Chem. B 110, 10170-10176 (2006).

Online Version 91

[24] S. Shinomoto and Y. Kuramoto, "Phase transitions in active rotator systems," Prog. Theo. Phys. 75, 1105-1110 (1986).

Online Version 92 


\section{Chapter 6}

\section{Phase Transition to Synchronization in}

\section{Globally Coupled Oscillators}

\subsection{Abstract}

In the natural world, periodic cycles determine individual and social behavior, often by governing activity in a manner crucial for survival. Synchronization of individuals in a population [1] is widely observed in chemical, biological, physical and social systems. Many such systems can be described as populations of coupled oscillators [2]. In Chapter 5, we discussed one type of transition to synchronization as quorum sensing, in which synchronization is triggered when the density of oscillators reaches a critical threshold [3]. This kind of sudden switch from one state to another has been found in yeast cells, which can switch from steady state to oscillatory behavior when the density of the cells reaches a critical threshold [4]. Another type of transition to is Kuramoto synchronization, in which unsynchronized oscillators gradually become synchronized as the coupling strength increases. The flashing 
of fireflies is a good example of this type of transition. In the classical Kuramoto synchronization, the frequency and phase of the oscillators become closer as the coupling strength is increased.

Experimental studies of photochemical oscillators [5] and electrochemical oscillators $[6,7]$ have shown more complex synchronization transitions. These are neither smooth, as in $\mathrm{Ku}-$ ramoto synchronization, nor a sudden switch, as in quorum sensing synchronization. In this synchronizing behavior, clusters of oscillators form in which frequency and phase can be synchronized but with different phases for different clusters. These clusters of oscillators are called phase clusters [8].

Oscillators have the same frequency and phase in the same cluster; however, in different clusters they may have different frequencies (frequency clusters) or different phases but the same frequency (phase clusters) [8]. Okuda has demonstrated that M phase clusters can be observed under certain condition, where $\mathrm{M}$ is the number of clusters [9]. The number of clusters and the configurations of oscillators can be predicted [10], and periodic oscillators with a distribution of natural frequencies can generate complex signals through global coupling [11]. Frequency heterogeneity is considered to be an important factor in multistability and formation of multiple clusters [10, 12].

Our research is based on large populations of chemical oscillators coupled by exchanging chemical intermediates through the surrounding solution, which spontaneously form phase clusters. Each cluster has the same frequency but a phase that is different with respect to the other clusters, such that the global signal generated by multiple clusters is more complex than that from a single cluster. We experimentally study the formation of phase clusters and their stability as a function of the density of the oscillators. We also simulate the cluster 
behavior based on the three-variable ZBKE model and compare our results with experiment.

\subsection{Experimental Setup}

\subsubsection{Particle Preparation}

In our experiment, we examine catalytic micro-particles immersed in a catalyst-free BZ solution. The chemical oscillators in our experiment are $\mathrm{Ru}(\mathrm{bpy})_{3}^{2+}$ coated microporous cation-exchange beads (DOWEX 50WX4-200). The beads, purchased from Fisher, are added to ruthenium bipyridil solution and stirred for 24 hours, allowing the $\mathrm{Ru}(\mathrm{bpy})_{3}^{2+}$ catalyst to be loaded onto the beads. $\mathrm{Ru}(\mathrm{bpy})_{3}^{2+}$ is the photosensitive catalyst for the $\mathrm{BZ}$ reaction and is widely used as a redox indicator, whose color characteristically changes between orange (the reduced form) and green (oxidized form) during the reaction. The beads are then filtered and washed with distilled water and then $0.1 \mathrm{M}$ sulfuric acid. The catalyst-coated beads are left to dry at room temperature in air for about 24 hours before being stored in a sealed bottle. The catalyst concentration on the beads is $1.65 \times 10^{-5} \mathrm{M}$. Figure 6.1 shows an image of catalytic micro-particales immersed in catalyst-free BZ solution.

We analyze beads loaded with the catalyst $\mathrm{Ru}(\mathrm{bpy})_{3}^{2+}$ and find that each bead has an oscillatory period dependent on its size and the catalyst loading. Figure 6.2 (a) shows an example of the periodic change in intensity of three individual beads, and Fig. 6.2 (b) shows the period distributions of the catalyst-loaded beads. The oscillatory period of the beads was measured in the unstirred BZ solutions, where the beads are arranged separately with at least three particle diameters between them. The concentrations of the reactants are 


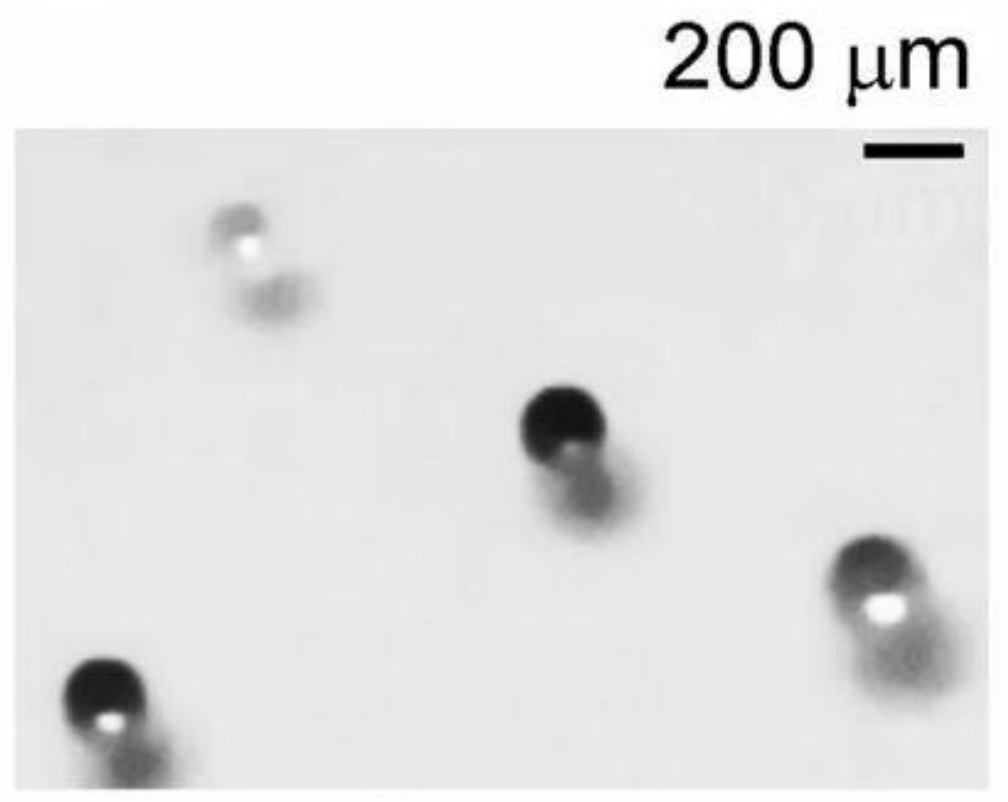

Figure 6.1. Image of catalytic micro-particles $\left(\mathrm{Ru}(\mathrm{bpy})_{3}^{2+}\right)$ in catalyst-free $\mathrm{BZ}$ solution. (Figure from reference [4].) 

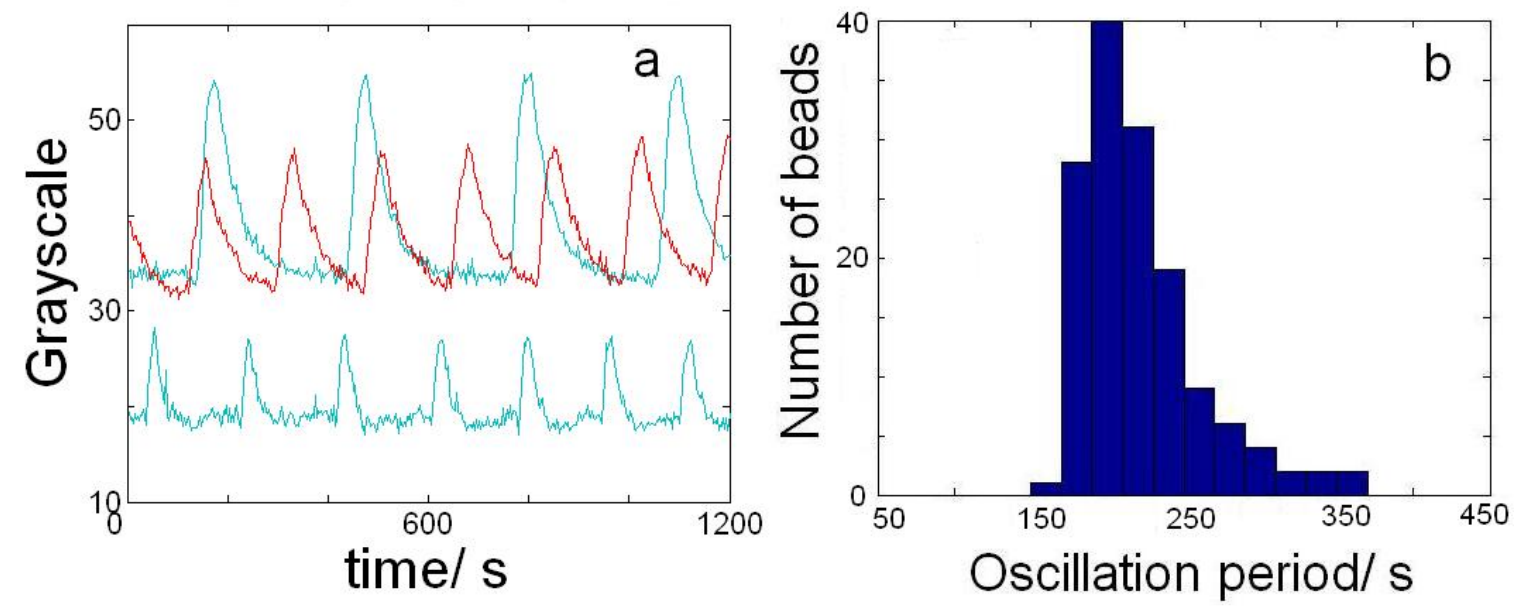

Figure 6.2. (a) Time series of the grayscale for three individual oscillatory beads. The concentrations of the solution: $\left[\mathrm{NaBrO}_{3}\right]=0.45 \mathrm{M},[\mathrm{MA}]=0.13 \mathrm{M},\left[\mathrm{H}_{2} \mathrm{SO}_{4}\right]=0.67 \mathrm{M}$ and $[\mathrm{NaBr}]=0.07 \mathrm{M}$. (b) The oscillation period distribution in a population of beads.

$\left[\mathrm{NaBrO}_{3}\right]=0.45 \mathrm{M},[\mathrm{MA}]=0.13 \mathrm{M},\left[\mathrm{H}_{2} \mathrm{SO}_{4}\right]=0.67 \mathrm{M}$ and $[\mathrm{NaBr}]=0.07 \mathrm{M}$. During the reaction, the color of catalyst-loaded beads changes between orange and green depending on the state of catalyst $\mathrm{Ru}(\mathrm{bpy})_{3}^{2+}$. The optical density of each bead can be recorded to track the oscillatory period of the bead.

\subsubsection{Instrumental Setup}

We use the same experimental setup as in our previous work. The reactor is a cylindrical vessel made of glass and fitted with a flat viewing window on the side. A redox Pt microelectrode (MI-800 from Microelectrodes, Inc.) is used to record the chemical electro-potential of the BZ solution, giving the average concentration of chemical species in the solution. A dig- 


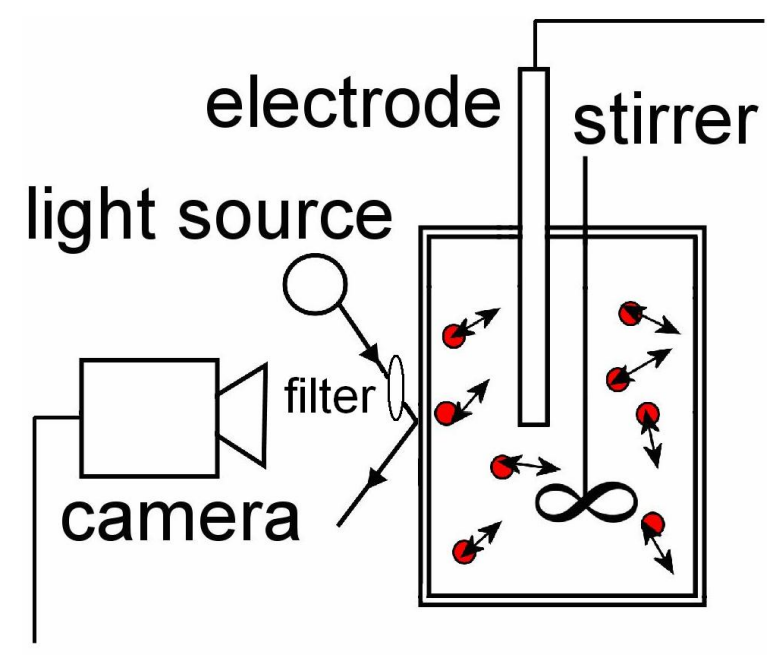

Figure 6.3. A sketch of the experimental setup.

ital camera (SPOT Insight IN1120, shutter speed $0.4 \mathrm{~ms}$ ) is used to photographically record the color change of the catalyst-loaded beads, allowing the oxidation state of the individual beads to be obtained from the images. The viewing window is illuminated by two fiber-optic lamps through a $480 \mathrm{~nm}$ low pass filter. $\mathrm{Ru}(\mathrm{bpy})_{3}^{2+}$ is photosensitive and has a maximum of absorbance at $454 \mathrm{~nm}$. A magnetic stirrer (IKAMAG) is with the stirring speed controlled at $600 \mathrm{rpm}$ to obtain well suspended particles in the BZ solution. The volume of the solution is $10.0 \mathrm{ml}$ and the solution temperature is $20^{\circ} \mathrm{C}$. Figure 6.3 shows a sketch of the experimental setup.

\subsection{Experimental Results}

From our previous work [3], we know that oscillators can synchronize their rhythms when the number density of particles is above a critical value in a well-stirred BZ solution. In this 
reaction system, two chemical species are provided by the catalytic beads to the surrounding solution, the inhibitor $\mathrm{Br}^{-}$and the activator $\mathrm{HBrO}_{2}$, and these species act as synchronizing agents. The ferroin catalyst used in previous work provided a smooth Kuramoto synchronization transition or a sudden quorum sensing transition to synchronization. With the catalyst $\mathrm{Ru}(\mathrm{bpy})_{3}^{2+}$ in place of ferroin, a more complex global signal in the surrounding solution is generated.

In our experiment, a period-2 signal (two signal peaks per oscillatory period) is observed in the electrical potential at low densities of beads. As the bead densities increase, the global signal initially is period-1 (one peak per oscillatory period) and then splits into two peaks with different amplitudes after several oscillations. With a further increase in the bead density, the period-1 oscillation can be maintained for a longer time before it splits into period-2 oscillations. Eventually, only period-1 oscillations exist for the global signal for high bead density. Figures 6.4 (i), (ii) and (iii) show the three electrical potential signals corresponding to three different bead densities.

There are two possibilities for explaining this behavior. One is that the oscillators develop their own complex behavior and then synchronize in the population. Another is that each oscillator keeps its original oscillatory state, and two groups of synchronized oscillators are formed that are separated by a phase difference.

Time series of the global electrode potential is recorded in Fig. 6.5 (i). We see that the frequency in the first $10 \mathrm{~min}$ is twice that of in the next $10 \mathrm{~min}$. The two types of oscillations, period-1 and period-2, are magnified in Fig. 6.5 (ii), and the optical intensity of the beads is shown in Fig. 6.5 (iii), obtained by analyzing the images. The calculation of bead intensity is the same as in Chapter 5.2.3. On the right side are eight sample images 


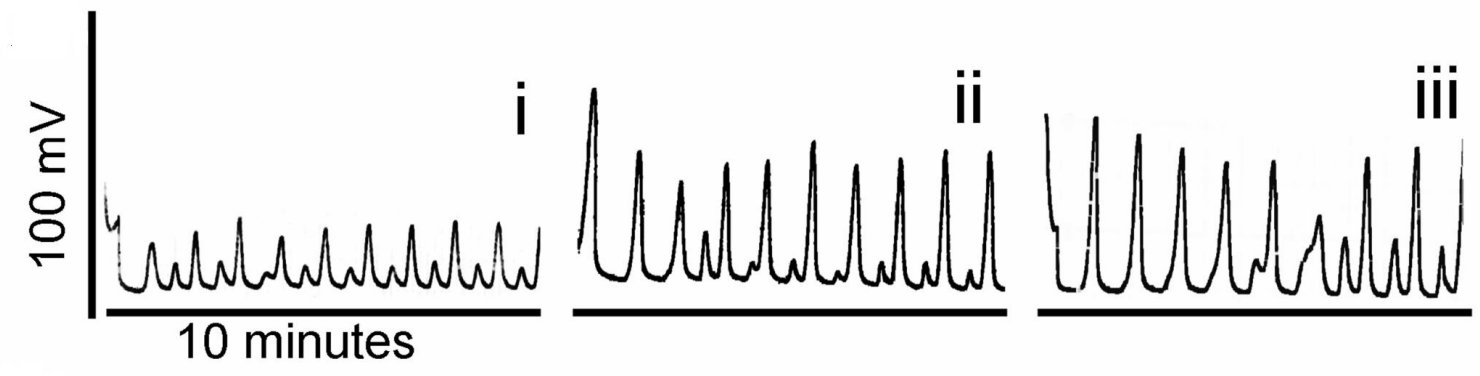

Figure 6.4. The electrode signals corresponding to three different bead densities. The concentrations of the solution: $\left[\mathrm{NaBrO}_{3}\right]=0.45 \mathrm{M},[\mathrm{MA}]=0.13 \mathrm{M},\left[\mathrm{H}_{2} \mathrm{SO}_{4}\right]=0.67 \mathrm{M}$ and $[\mathrm{NaBr}]=0.07$ M. (i) The bead density $\mathrm{n}=0.03 \mathrm{~g} \mathrm{ml}^{-1}$, (ii) $\mathrm{n}=0.032 \mathrm{~g} \mathrm{ml}^{-1}$, (iii) $\mathrm{n}=$ $0.04 \mathrm{~g} \mathrm{ml}^{-1}$. The time for maintaining the period-1 oscillation increases as the bead density increases. (Figure reproduced from reference [4].)

which were captured at the times marked in Fig. 6.5 (iii).

\subsection{Numerical Simulations}

\subsubsection{ZBKE Model of Ruthenium Catalysed Particle System}

The ZBKE model described in Chapter 5 provides a four-variable system of differential equations to simulate the catalyst-particle system. The model consists of the rate equations for the individual beads:

$$
\begin{aligned}
\frac{d X_{i}}{d t}= & -k_{e x}\left(X_{i}-X_{s}\right)-k_{2} h_{0} X_{i} Y_{i}+k_{3} h_{0} A Y_{i}-2 k_{4} X_{i}^{2}-k_{5} h_{0} A X_{i} \\
& +k_{-5} U_{s s}^{2}+k_{6} U_{s s}\left(C-Z_{i}\right),
\end{aligned}
$$



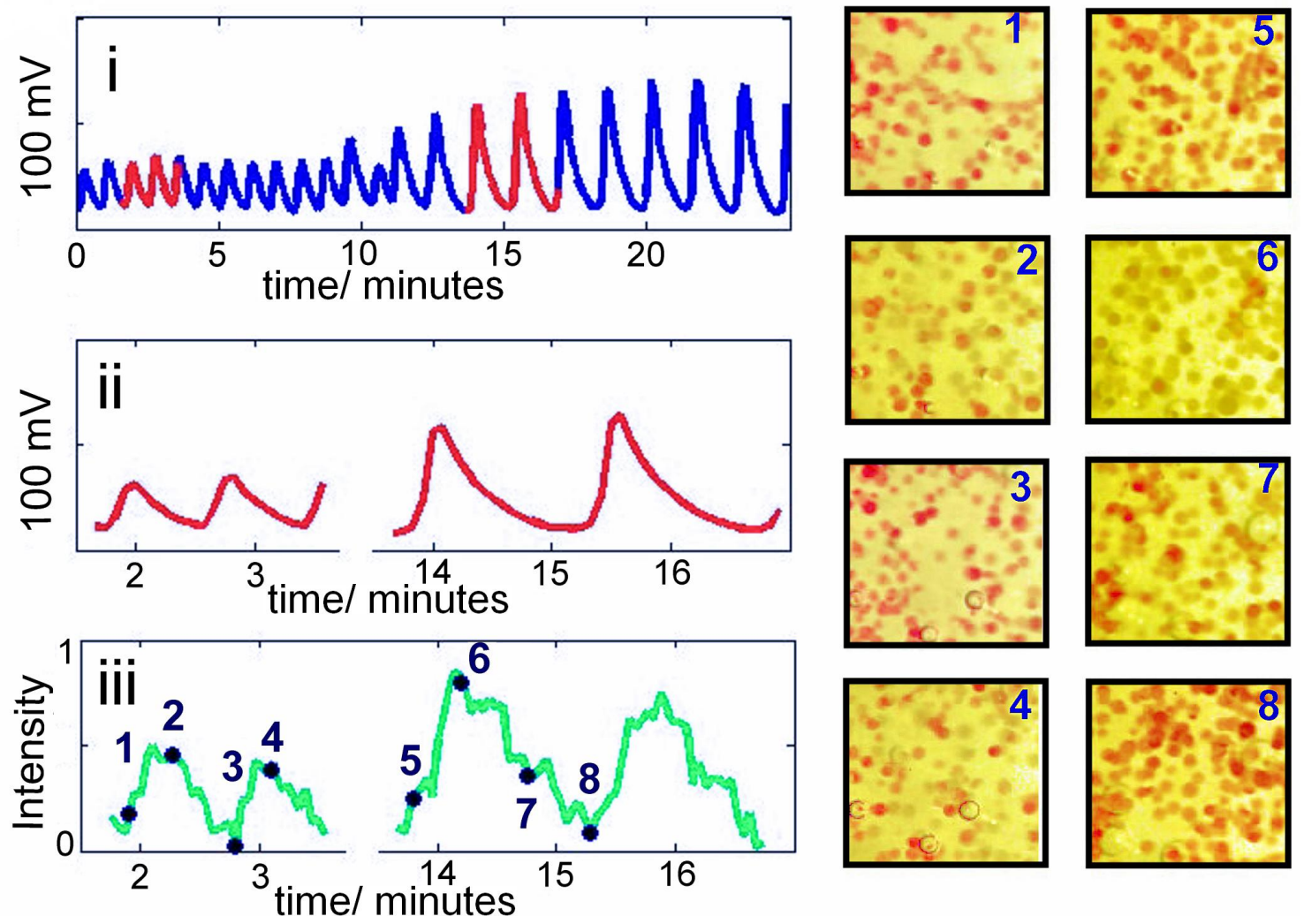

Figure 6.5. The electrode potential signal and sample images for one experiment. The bead density of the experiment $\mathrm{n}=0.026 \mathrm{~g} \mathrm{ml}^{-1}$. The concentrations of the solution: $\left[\mathrm{NaBrO}_{3}\right]$ $=0.45 \mathrm{M},[\mathrm{MA}]=0.13 \mathrm{M},\left[\mathrm{H}_{2} \mathrm{SO}_{4}\right]=0.67 \mathrm{M}$ and $[\mathrm{NaBr}]=0.07 \mathrm{M}$. (i) The electrode signal trace for the whole time series. (ii) Magnified signal trace corresponding to the red area in (i). (iii) The intensity of beads corresponding to the same time series of (ii). Eight sample images are shown on right side. The time of each image is marked in (iii). The image area is about $2 \times 2 \mathrm{~mm}^{2}$. (Figure reproduced from reference [4].) 


$$
\begin{gathered}
\frac{d Y_{i}}{d t}=-k_{e x}\left(Y_{i}-Y_{s}\right)-k_{2} h_{0} X_{i} Y_{i}-k_{3} h_{0} A Y_{i}+q_{i} \frac{k_{7} k_{8} B Z_{i}}{k_{-7} h_{0}\left(C-Z_{i}\right)+k_{8}}+k_{9} B, \\
\frac{d Z_{i}}{d t}=k_{6} U_{s s}\left(C-Z_{i}\right)-\frac{k_{7} k_{8} B Z_{i}}{k_{-7} h_{0}\left(C-Z_{i}\right)+k_{8}} .
\end{gathered}
$$

Here, $X, Y$ and $Z$ represent the concentrations of the activator $\mathrm{HBrO}_{2}$, the inhibitor $\mathrm{Br}^{-}$, and the oxidized form of catalyst $\mathrm{Ru}(\mathrm{bpy})_{3}^{2+} . X_{i}, Y_{i}$ and $Z_{i}$ are the concentrations of the species for each particle $(i=1, \ldots N)$. The intermediate $\mathrm{HBrO}_{2}^{+}(U)$ is given by its steadystate concentration $\left(U_{s s}\right)$ :

$$
U_{s s}=\frac{1}{4 k_{-5}}\left(-k_{6}\left(C-Z_{i}\right)+\left(k_{6}^{2}\left(C-Z_{i}^{2}\right)+16 k_{-5} k_{5} h_{0} A x_{i}+8 k_{-5} k_{-6} x_{i} z_{i}\right)^{1 / 2}\right) .
$$

In the surrounding solution, the concentrations of $\mathrm{HBrO}_{2}$ and $\mathrm{Br}^{-}\left(X_{s}\right.$ and $\left.Y_{s}\right)$ are:

$$
\begin{gathered}
\frac{d X_{s}}{d t}=\frac{\bar{V}}{V_{s}} \sum_{i=1}^{N} k_{e x}\left(X_{i}-X_{s}\right)-k_{2} h_{0} X_{s} Y_{s}+k_{3} h_{0} A Y_{s}-2 k_{4} X_{s}^{2}+4 k_{-5} k_{5} h_{0} A X_{s}, \\
\frac{d Y_{s}}{d t}=\frac{\bar{V}}{V_{s}} \sum_{i}^{N} k_{e x}\left(Y_{i}-Y_{s}\right)-k_{2} h_{0} X_{s} Y_{s}-k_{3} h_{0} A Y_{s}+k_{9} B .
\end{gathered}
$$

$\mathrm{A}=\left[\mathrm{HBrO}_{3}\right], \mathrm{B}=\left[\mathrm{CHBr}(\mathrm{COOH})_{2}\right]+\left[\mathrm{CH}_{2}(\mathrm{COOH})_{2}\right], \mathrm{C}=$ total catalyst, $\mathrm{h}_{0}=$ Hammet acidity function, $q_{i}=$ stoichiometric coefficient, $k_{2} \sim k_{9}=$ rate constant, $k_{e x}=$ exchange rate constant, $V_{s}=$ total volume of the solution, $\bar{V}=$ average volume of single bead. All of the parameters used in the simulations for the ruthenium-catalyzed BZ system are listed in Table 6.1.

\subsubsection{Comparison of Ruthenium and Ferroin Catalysed BZ Reaction}

Comparing the three-variable ZBKE model for $\mathrm{Ru}(\mathrm{bpy})_{3}^{2+}$ with the ferroin-catalyzed ZBKE model, we see the change of $\mathrm{HBrO}_{2}$ and $\mathrm{Br}^{-}$in Figs. 6.6 (a) (i) and 6.6 (b) (i). 
Table 6.1. The parameter values in the ruthenium-catalyzed ZBKE model [13]

\begin{tabular}{|c|c|c|c|c|c|}
\hline Parameter & Value & Parameter & Value & Parameter & Value \\
\hline $\mathrm{A}(\mathrm{M})$ & 0.51 & $k_{2}\left(\mathrm{M}^{-2} \mathrm{~s}^{-1}\right)$ & $2 \times 10^{6}$ & $k_{-6}\left(\mathrm{M}^{-1} \mathrm{~s}^{-1}\right)$ & 0 \\
$\mathrm{~B}(\mathrm{M})$ & 0.16 & $k_{3}\left(\mathrm{M}^{-2} \mathrm{~s}^{-1}\right)$ & 2.0 & $k_{8} / k_{-7}\left(\mathrm{M}^{2}\right)$ & $2.5 \times 10^{-4}$ \\
$\mathrm{C}(\mathrm{M})$ & variable & $k_{4}\left(\mathrm{M}^{-1} \mathrm{~s}^{-1}\right)$ & 3000 & $k_{8} k_{7} / k_{-7}\left(\mathrm{M} \mathrm{s}^{-1}\right)$ & $1.4 \times 10^{-2}$ \\
$h_{0}$ & 0.77 & $k_{5}\left(\mathrm{M}^{-2} \mathrm{~s}^{-1}\right)$ & 33 & $k_{9}\left(\mathrm{~s}^{-1}\right)$ & $3.0 \times 10^{-6}$ \\
$\bar{q}$ & 0.8 & $k_{-5}\left(\mathrm{M}^{-1} \mathrm{~s}^{-1}\right)$ & $4.2 \times 10^{6}$ & $k_{e x}\left(\mathrm{~s}^{-1}\right)$ & 0.9 \\
$\sigma_{q}$ & 0.1 & $k_{6}\left(\mathrm{M}^{-1} \mathrm{~s}^{-1}\right)$ & $4 \times 10^{7}$ & $\bar{V} / V_{s}$ & variable \\
\hline
\end{tabular}

Figures 6.6 (a) (ii) and 6.6 (b) (ii) show the corresponding phase response for $\mathrm{HBrO}_{2}$ or $\mathrm{Br}^{-}$ perturbations. From the figure, we see that the $\mathrm{Br}^{-}$perturbation causes mainly a negative phase shift, which means that pulses of $\mathrm{Br}^{-}$induce phase delays, while $\mathrm{HBrO}_{2}$ perturbations mainly cause positive phase shifts that induce phase advances for the next oscillation.

\subsubsection{Formation of Clusters}

In Fig. 6.7, we simulate the experimental behavior shown in Fig. 6.4, based on the ZBKE model. The initial single peak splits to form small peaks in the oscillatory trace. The amplitude of $\mathrm{Br}^{-}$concentration in the surrounding solution increases with a number density increase, and the time before the spliting occurs becomes longer.

In our simulations, the individual oscillators are analyzed to interpret the cluster formation. Figure 6.8 (i) shows the time series of bromide concentration in the surrounding 

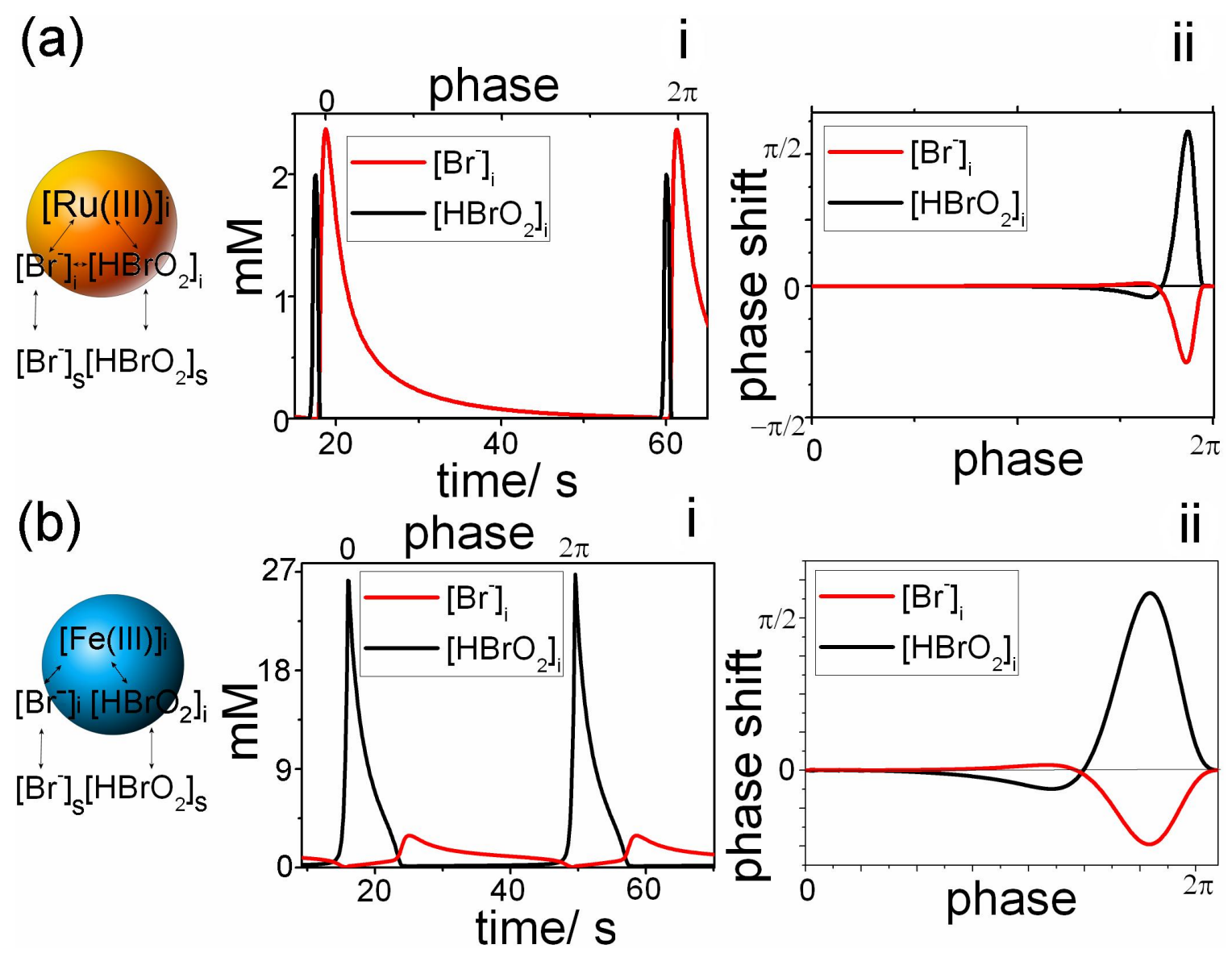

Figure 6.6. Simulations of two species $\left(\mathrm{HBrO}_{2}\right.$ and $\left.\mathrm{Br}^{-}\right)$behavior in $\mathrm{Ru}(\mathrm{bpy})_{3}^{2+}$ (a) and ferroin (b) catalysed BZ reaction system. The mass exchange to the surrounding solution is zero. The $\mathrm{Ru}(\mathrm{bpy})_{3}^{2+}$ coated particle (orange), and the ferroin coated particle (blue) are shown at left. (i) Oscillatory behavior of $\mathrm{HBrO}_{2}$ and $\mathrm{Br}^{-}$is shown in (a) (i) and (b) (i) for the $\mathrm{Ru}(\mathrm{bpy})_{3}^{2+}$ and ferroin systems, respectively. (ii) The corresponding phase response following $\mathrm{HBrO}_{2}$ and $\mathrm{Br}^{-}$perturbations. The phase is given as: $\phi(t)=2 \pi\left(t-T_{n}\right) /\left(T_{(n+1)}-T_{n}\right)$, where $\mathrm{T}$ is the period with the perturbation. (Figure from reference [4].) 


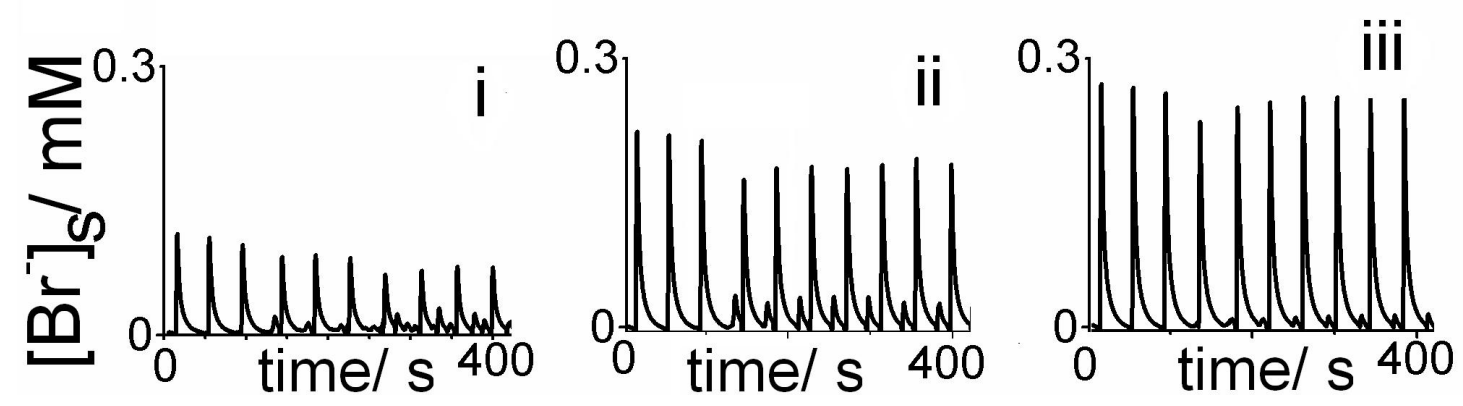

Figure 6.7. Simulations of cluster formation. Y axis represents the bromide concentration in the surrounding solution. The catalyst concentration $\mathrm{C}=3.3 \times 10^{-2} \mathrm{M}$. (i) The number density $\mathrm{n}=9.5 \times 10^{3} \mathrm{~cm}^{-3}$, (ii) $\mathrm{n}=1.9 \times 10^{4} \mathrm{~cm}^{-3}$ and (iii) $\mathrm{n}=2.4 \times 10^{4} \mathrm{~cm}^{-3}$. (Figure reproduced from reference [4].)

solution and Fig. 6.8 (iii) shows the bromide concentration traces on four individual oscillators. The bromide concentration of each oscillator is known as a function of time, and the corresponding phase function therefore can be $\operatorname{constructed.~We~use~} \cos \theta$ vs $\sin \theta$ to describe the phase, and the result is a phase plot of the oscillators moving around the cycle with constant velocity. In Fig. 6.8 (iii), the four oscillators are initially at the same phase at $\mathrm{t}$ $=98 \mathrm{~s}$. The oscillator with the shorter natural period arrives at its peak value of bromide concentration first (the blue trace). This causes an increase in the bromide concentration in the surrounding solution, affecting all other oscillators. This bromide perturbation causes a phase delay of the other oscillators which then form a second group. In the next oscillatory cycle, the light blue oscillator falls back and joins the second group. Figure 6.8 (ii) shows the period distribution after the first split. The small group period is around $40 \mathrm{~s}$ and the large group period is around $50 \mathrm{~s}$. There are 1000 oscillators in the system. 
From experiments, we find that the amplitude of the electrode potential either remains stable or changes with time, as shown in Figs. 6.9 (a) (i) and 6.9 (a) (ii). This behavior arises because the oscillators either have a stable group configuration or an unstable group configuration. If a fraction of the oscillators can jump between groups, it will cause changes in the amplitude of the global signal. In the simulation, using the same parameters and slightly different natural periods of oscillators, we observe these two kinds of behavior for period-2 phase clusters. One is a stable configuration, shown in Fig. 6.9 (b) (i), and the other changes with time, shown in Fig. 6.9 (b) (ii). In Fig. 6.9 (b) (ii), we see that the amplitude of the trace varies with time, which means the configuration of oscillators in the two groups is unstable and some oscillators jump between the two groups. Traces of the bromide concentration on 100 sample oscillators and their phase cycle are shown, representing these two configurations. The jump of the oscillators is due to the phase delay arising from the $\mathrm{Br}^{-}$perturbation or the phase advance resulting from the $\mathrm{HBrO}_{2}$ perturbation.

\subsubsection{Factors Influencing Cluster Formation}

We know that the concentrations of $\mathrm{HBrO}_{2}$ and $\mathrm{Br}^{-}$play an important role in cluster formation, and that the number of clusters is a function of the number density. Figure 6.10(a) shows that the number of clusters decreases as the density is increased. When the number density increases to a threshold value, there exist only one cluster in the system. In simulations, we have observed up to four clusters in the system, but only one and two clusters have been observed in our experiments. The reason is that one and two clusters 


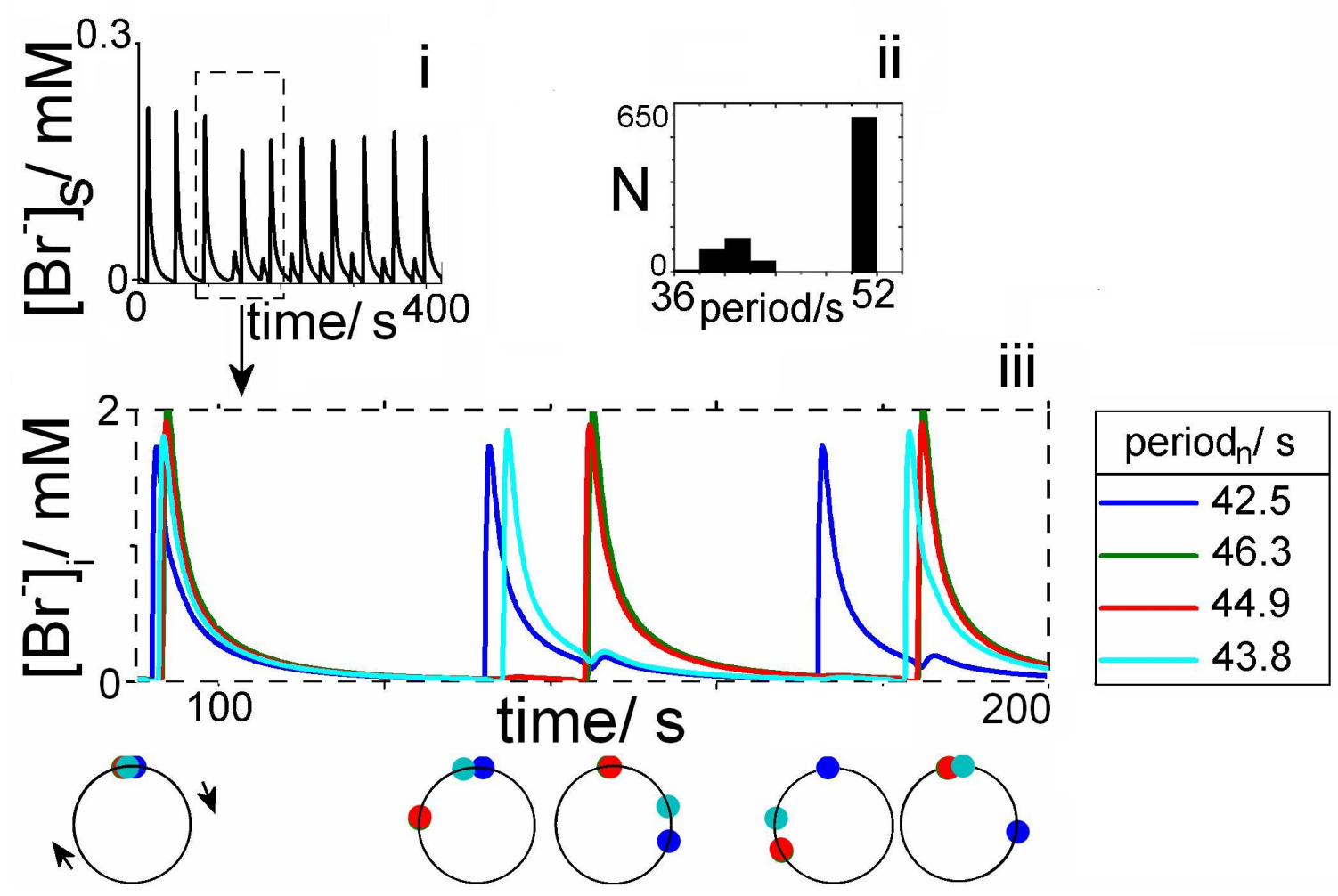

Figure 6.8. Simulations and interpretation of phase cluster formation. The catalyst concentration $\mathrm{C}=3.3 \times 10^{-2} \mathrm{M}$. The number density $\mathrm{n}=1.9 \times 10^{4} \mathrm{~cm}^{-3}$. (i) Time series of bromide concentration in the surrounding solution. (ii) The period distribution after the initial split of the total number of oscillators $(\mathrm{N}=1000)$. (iii) The bromide concentration traces on four individual oscillators corresponding to the trace in (i), and the corresponding the phase plots below. The natural periods of the four individual oscillators are shown on the right. (Figure reproduced from reference [4].) 
(a)

\section{experiment}

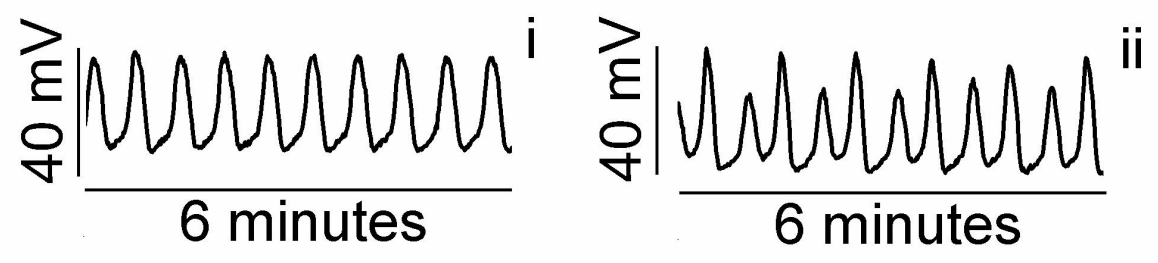

(b)

simulation

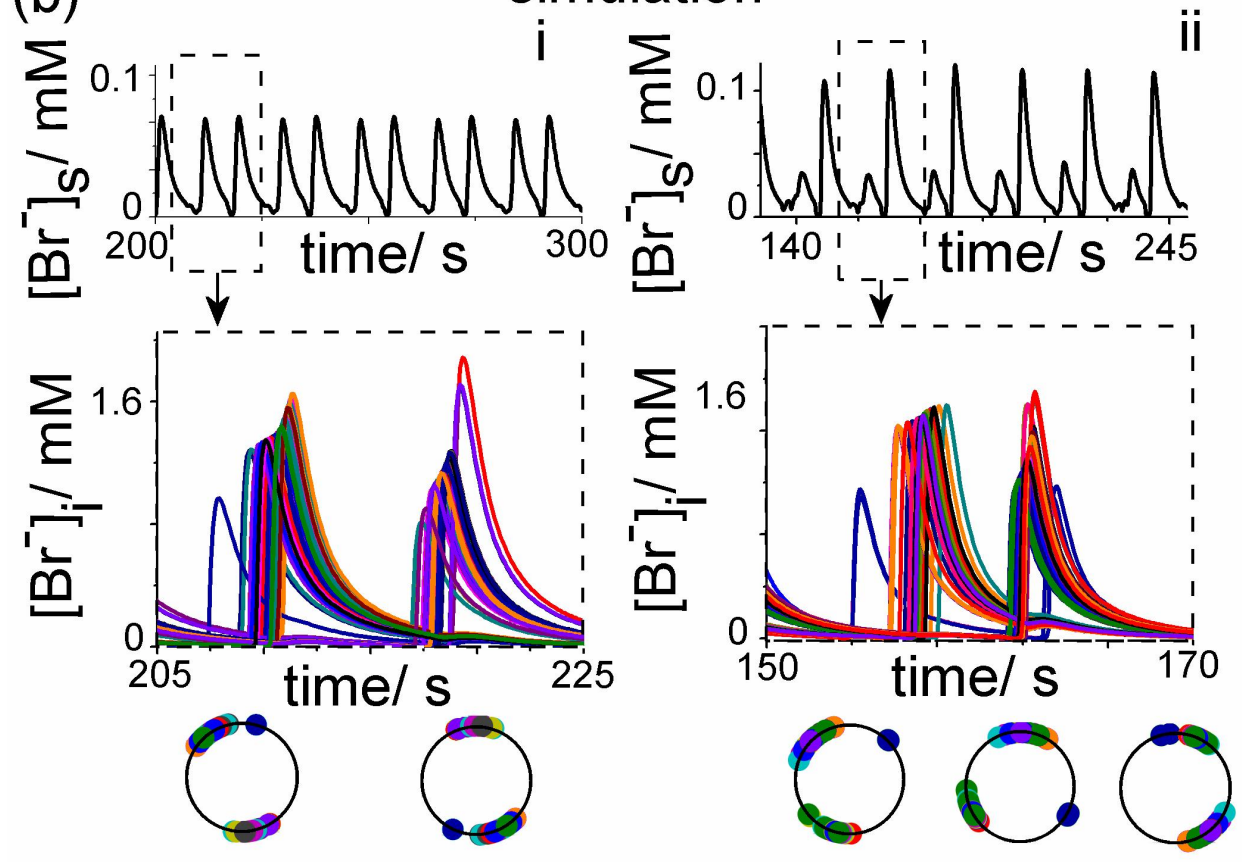

Figure 6.9. Switching oscillators in experiments and simulations. (a) Time series of electrical potential signal of period-2 oscillations. Number density $\mathrm{n}=0.02 \mathrm{~g} \mathrm{ml}^{-1}$ : (i) stable signal, (ii) unstable signal, and (b) simulation results. Total number of oscillators $\mathrm{N}=1000$. (i) Stable configuratiion of oscillators: the amplitude of bromide concentration in the surrounding solution is stable in time. (ii) Unstable configuration of oscillators: the amplitude of bromide concentration in the surrounding solution is changing in time. The traces of bromide comcentration on 100 sample oscillators and their phase cycle are shown in the lower plots. In (b) (ii), the blue oscillator at the front group jumps and joins the other group, which causes the amplitude in the global signal to change. (Figure from reference [4].) 
have a wide rage of number density compared to three and four clusters. If the exchange of $\mathrm{HBrO}_{2}$ does not occur $\left(k_{e x}=0\right.$ for $\left.\mathrm{HBrO}_{2}\right)$, the number of clusters decreases more slowly with increasing density, as shown in Fig. 6.10 (b). If the exchange of $\mathrm{Br}^{-}$does not occur $\left(k_{e x}=0\right.$ for $\left.\mathrm{Br}^{-}\right)$, only one cluster exists for all number densities, as shown in Fig. 6.10 (c). Only one cluster exists if there is no $\mathrm{HBrO}_{2}$ and $\mathrm{Br}^{-}$exchange in the surrounding solution, as shown in Fig. 6.10 (d). Both are synchronizing species, and their perturbations either advances or delays the phase of the oscillators.

We find that the coupling strength $k_{e x}$ is another important factor that affects the number of clusters. Figure 6.11 shows the formation of different numbers of clusters under different coupling strengths when the number density remains constant. The number of clusters increases with decreasing clupling strength $k_{e x}$, and only one cluster exists at high

coupling strength. At low stirring speed (around $600 \mathrm{rpm}$ ), a period-2 electrochemical signal is observed; however, when the stirring speed is increased to $900 \mathrm{rpm}$, only a period-1 signal is observed.

\subsection{Summary}

Cluster formation in globally coupled oscillators has been experimentally observed using the electrode potential signal and intensity of beads from the captured images. Period-2 electrochemical signals and intensity oscillations have been observed. We also found that the amplitude of the signal and the time elapsed before the initial split are related to the number density of the oscillators. 
(a)

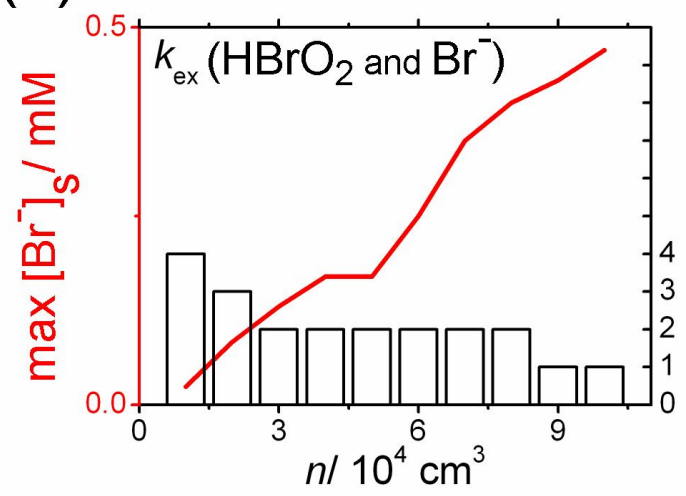

(c)

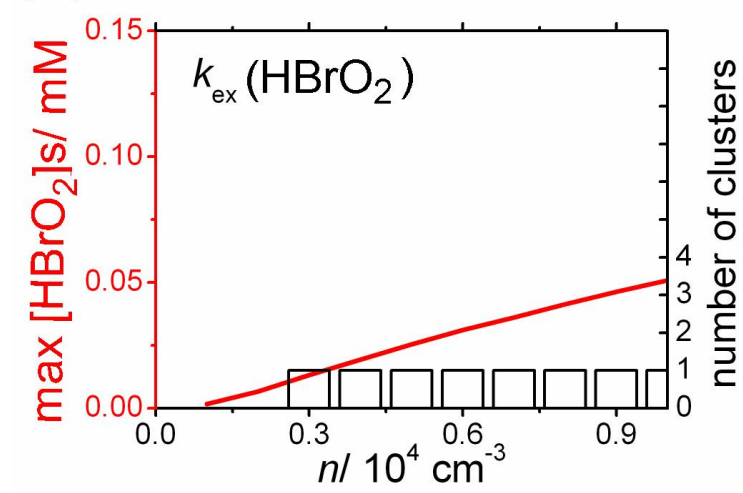

(b)

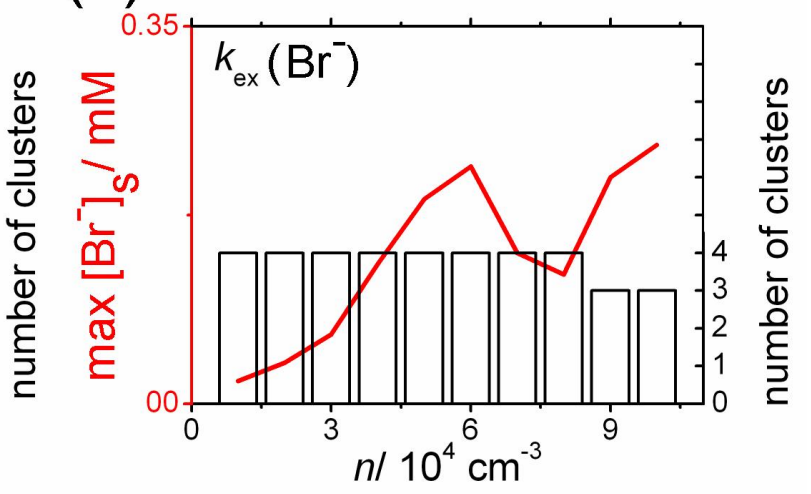

(d)

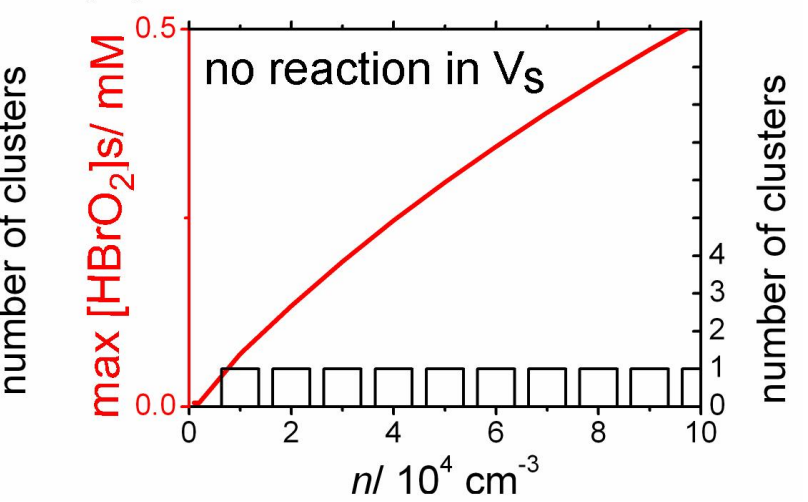

Figure 6.10. Simulated behavior of $\mathrm{HBrO}_{2}$ and $\mathrm{Br}^{-}$concentration in the surrounding solution and the cluster number as a function of the number density. (a) Both $\mathrm{HBrO}_{2}$ or $\mathrm{Br}^{-}$exchange occurs with the surrounding solution, (b) only $\mathrm{Br}^{-}$exchange occurs, (c) only $\mathrm{HBrO}_{2}$ exchange occurs, and (d) no exchange occurs. (Figure from reference [4].) 

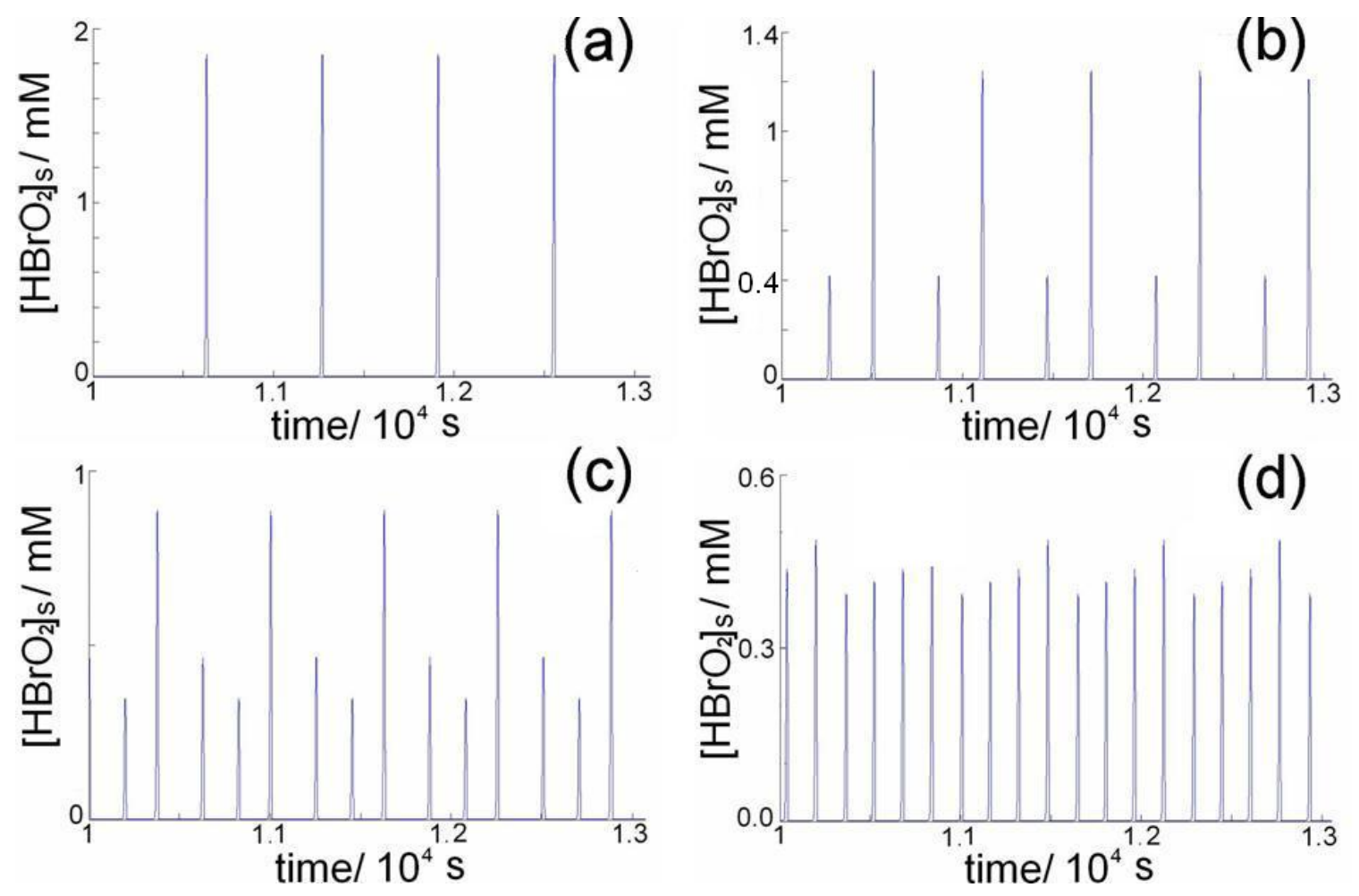

Figure 6.11. Simulations of the $\mathrm{HBrO}_{2}$ concentration in the surrounding solution at different coupling strength $k_{e x}$. The number density of oscillators $\mathrm{n}=1400 \mathrm{~cm}^{-3}$. (a) At a coupling strength $k_{e x}=1.5$, only one cluster is observed, (b) with $k_{e x}=1.1$, two clusters are formed, (c) with $k_{e x}=0.9$, three clusters are formed, (d) and with $k_{e x}=0.5$, four clusters are formed. 
Based on ZBKE model [13], we set up a three-variable ZBKE model for the rutheniumcatalysed particle system. We have simulated the $\mathrm{HBrO}_{2}$ and $\mathrm{Br}^{-}$concentrations on the individual oscillators and in the surrounding solution. By analyzing the behavior of individual oscillators, the cluster formation can be interpreted in detail. An $\mathrm{HBrO}_{2}$ perturbation causes a phase advance and a $\mathrm{Br}^{-}$perturbation causes a phase delay. Consequently, phase clusters are formed and oscillators can jump between the clusters. We have also demonstrated that the number of clusters is related to the number density $\mathrm{n}$ and coupling strength $k_{e x}$. The cluster number decreases as the number density increases with $\mathrm{HBrO}_{2}$ and $\mathrm{Br}^{-}$exchange in the surrounding solution. The same trends are observed for the coupling strength $k_{e x}$. 


\section{Reference}

[1] J. A. Acebrón, L. L. Bonilla, C. J. P. Vicente, F. Ritort, and R. Spigler, "The Kuramoto model: A simple paradigm for synchronization phenomena," Rev. Mod. Phys. 77, 137$185(2005)$.

Online Version 104

[2] Y. Kuramoto, Chemical oscillations, waves, and turbulence (Springer-Verlag, New York, 1984). 104

[3] A. F. Taylor, M. Tinsley, F. Wang, Z. Huang, and K. Showalter, "Dynamical quorum sensing and synchronization in large populations of chemical oscillators," Science 323, 614-617 (2009).

Online Version 104, 109

[4] A. F. Taylor, M. Tinsley, F. Wang, and K. Showalter, "Phase clusters in large populations of chemical oscillators," accepted by Angewandte Chemie (2011). 104, 107, 111, $112,115,116,118,119,121$

[5] A. F. Taylor, P. Kapetanopoulos, B. J. Whitaker, R. Toth, L. Bull, and M. R. Tinsley, "Clusters and switchers in globally coupled photochemical oscillators," Phys. Rev. Lett. 100, 214101-1-4 (2008).

Online Version 105

[6] I. Z. Kiss, C. G. Rusin, H. Kori, and J. L. Hudson, "Engineering complex dynamical structures: Sequential patterns and desynchronization," Science 316, 1886-1889 (2007). Online Version 105 
[7] I. Z. Kiss, Y. M. Zhai, and J. L. Hudson, "Characteristics of cluster formation in a population of globally coupled electrochemical oscillators: An experiment-based phase model approach," Prog. Theor. Phys. Supp. 161, 99-106 (2006).

Online Version 105

[8] D. Golomb, D. Hansel, B. Shraiman, and H. Sompolinsky, "Clustering in globally coupled phase oscillators," Phys. Lett. A 45, 3516-3530 (1992).

Online Version 105

[9] K. Okuda, "Variety and generality of clustering in globally coupled oscillators," Physica D 63, 424-436 (1993).

Online Version 105

[10] P. Tass, "Phase and frequency shifts in a population of phase oscillators," Phys. Rev. E 56, 2043-2060 (1997).

Online Version 105

[11] H. Daido, "Multi-branch entrainment and multi-peaked order-functions in a phase model of limit-cycle oscillators with uniform all-to-all coupling," J. Phys. A: Math. Gen. 28, L151-L157 (1995).

Online Version 105

[12] Z. G. Zheng, G. Hu, and B. Hu, "Phase slips and phase synchronization of coupled oscillators," Phys. Rev. Lett. 81, 5318-5321 (1998).

Online Version 105

[13] A. M. Zhabotinsky, F. Buchholtz, A. B. Kiyatkin, and I. R. Epstein, "Oscillations and 125 
waves in metal-ion-catalyzed bromate oscillating reactions in highly oxidized states," J.

Phys. Chem. 97, 1518-7584 (1993).

Online Version ix, 114, 123

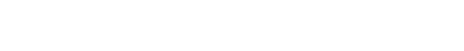

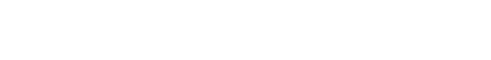

Virginia University Libraries,

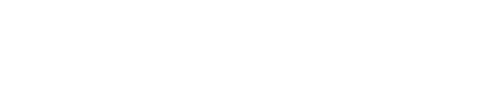

